\title{
Teoria de Ramsey \\ para circuitos e caminhos
}

\author{
Fabricio Siqueira Benevides
}

DissertaÇÃo Apresentada

$\mathrm{AO}$

Instituto DE MATEMÁtica e EstatísticA

DA

UNIVERSIDADE DE SÃO PAULO

PARA

ObtençÃo de Título de Mestre

EM

CIÊNCIAS

Área de concentração: Ciências da Computação

Orientador: Prof. Dr. Yoshiharu Kohayakawa

Durante a elaboração deste trabalho o autor recebeu auxílio financeiro do CNPq e da Fapesp

São Paulo, fevereiro de 2007 



\section{Teoria de Ramsey para circuitos e caminhos}

Este exemplar corresponde à redação final da dissertação devidamente corrigida e defendida por Fabricio Siqueira Benevides e aprovada pela comissão julgadora.

São Paulo, 10 de junho de 2007

Banca examinadora:

Prof. Dr. Yoshiharu Kohayakawa (presidente)

IME-USP

Prof. Dr. Carlos Gustavo Tamm de Araujo Moreira

IMPA

Prof. Dr. Paulo Feofiloff

IME-USP 



\section{Agradecimentos}

A Deus, por colocar as pessoas certas em meu caminho e por me emprestar todas as ferramentas necessárias ao meu desenvolvimento intelectual e moral.

Ao Professor Dr. Yoshiharu Kohayakawa, pela orientação segura, sem a qual não seria possível a realização deste trabalho.

Ao Professor Dr. Jozef Skokan, pelo incentivo na fase final deste trabalho e pelos valorosos "insights".

Ao Professor Dr. Paulo Feofiloff e aos demais membros da banca pela cuidadosa leitura dessa dissertação e pelos valorosos conselhos que vieram a melhorar significantemente o texto.

À Fundação de Amparo à Pesquisa do Estado de São Paulo (FAPESP) e ao Conselho Nacional de Desenvolvimento Científico e Tecnológico (CNPq), pelas concessões das bolsas de estudo para a realização deste trabalho.

Aos meus colegas de turma do Instituto de Matemática e Estatística da Universidade de São Paulo, pela calorosa receptividade e pelos bons momentos em que estivemos juntos. Vocês realmente me ajudaram muito nessa caminhada.

Gostaria de agradecer também a toda minha família por me proporcionar o ambiente de amor onde tive a sorte de crescer. Meus irmãos, cunhada e sobrinhos foram particularmente importantes.

Também sou grato especialmente ao meu irmão e à minha irmã por sempre terem sido exemplos de vida para mim.

Finalmente, Estarei em débito eterno com meus pais e com Juliana pela compreensão, paciência interminável e encorajamentos quando estes eram mais necessários. Para eles, eu dedico esta dissertação. 



\section{Resumo}

Os principais objetos de estudo neste trabalho são os números de Ramsey para circuitos e o lema da regularidade de Szemerédi. Dados grafos $L_{1}, \ldots, L_{k}$, o número de Ramsey $R\left(L_{1}, \ldots, L_{k}\right)$ é o menor inteiro $N$ tal que, para qualquer coloração com $k$ cores das arestas do grafo completo com $N$ vértices, existe uma cor $i$ para a qual a classe de cor correspondente contém $L_{i}$ como um subgrafo. Estaremos especialmente interessados no caso em que os grafos $L_{i}$ são circuitos.

Diversos resultados sobre este tema surgiram na última década. No capítulo 1, enunciamos alguns desses resultados, incluindo um resultado original. No capítulo 2, apresentamos um breve histórico sobre teoria de Ramsey e todas as definições básicas necessárias para aqueles que não estão familiarizados com a área. Em seguida, introduzimos o lema da regularidade e exibimos uma aplicação deste lema em um problema em que temos interesse. No capítulo 4, exibimos uma prova detalhada de um recente lema de estabilidade sobre emparelhamentos grandes em 3-colorações de grafos quase completos. Este lema será necessário na prova do resultado principal do capítulo 5, que é o principal fruto deste trabalho. 



\section{Abstract}

The main objects of interest in this work are the Ramsey numbers for cycles and the Szemerédi regularity lemma. For graphs $L_{1}, \ldots, L_{k}$, the Ramsey number $R\left(L_{1}, \ldots, L_{k}\right)$ is the minimum integer $N$ such that for any edge-coloring of the complete graph with $N$ vertices by $k$ colors there exists a color $i$ for which the corresponding color class contains $L_{i}$ as a subgraph. We are specially interested in the case where the graphs $L_{i}$ are cycles.

Many results about this theme were obtained in the last decade. In chapter 1 , we enumerate some of these results, including an original one. In chapter 2 , we state the basic definitions, specially for those who are not familiar with this subject, and give an historical overview of Ramsey theory itself. In the next chapter, we introduce the regularity lemma and we exhibit an application of this lemma in a problem of our interest. In chapter 4, we show a detailed proof of a very recent stability lemma about large matchings in a 3-coloring of an almost complete graph. This lemma will be necessary in the proof of the main result of chapter 5 , which is the main product of this work. 



\section{Sumário}

Agradecimentos v v

Resumo vii

Abstract vii

1 Introdução 1

2 Conceitos básicos e histórico 5

2.1 Breve histórico da teoria de Ramsey . . . . . . . . . . . . . . . . . . . . . . . 5

2.2 Colorindo arestas de grafos . . . . . . . . . . . . . . . . 8

2.3 Ramsey para grafos . . . . . . . . . . . . . . . . . . . . . . . . . . . . . . . . . . . . . . . . . .

2.4 Teoremas de densidade . . . . . . . . . . . . . . . . . . . . . . . 14

$\begin{array}{lll}3 & \text { Lema da regularidade } & 17\end{array}$

3.1 Uma aplicação do lema da regularidade . . . . . . . . . . . . . . . 22

4 Um lema de estabilidade $\quad 25$

4.1 Ferramentas e resultados do tipo Ramsey . . . . . . . . . . . . . . 28

4.2 Prova do lema $4.1 \ldots \ldots \ldots$. . . . . . . . . . . . . . . . . 34

4.2.1 Caso 1 da prova: existe uma 4-partição . . . . . . . . . . . 37

4.2.2 Caso 2 da prova: existe uma componente monocromática grande 40

4.2.3 Prova do lema 4.11: reduzindo colorações extremais fracas . . . . 51

5 Ramsey para circuitos pares $\quad \mathbf{5 7}$

5.1 Prova do teorema . . . . . . . . . . . . . . . . . . . 59

5.2 Caminhos e circuitos em grafos densos . . . . . . . . . . . . 62

5.3 Prova dos lemas 5.3 e $5.5 \ldots \ldots \ldots \ldots$. . . . . . . . . . . 65

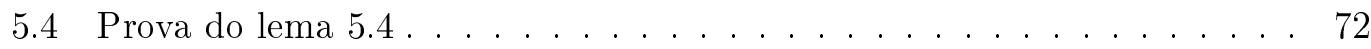

$\begin{array}{ll}\text { Bibliografia } & 75\end{array}$ 



\section{Capítulo 1}

\section{Introdução}

Dados grafos $L_{1}, \ldots, L_{k}$, o número de Ramsey $R\left(L_{1}, \ldots, L_{k}\right)$ é o menor inteiro $N$ tal que, para qualquer coloração com $k$ cores das arestas do grafo completo com $N$ vértices, existe uma cor $i$ para a qual a classe de cor correspondente contém $L_{i}$ como subgrafo. Começamos exibindo alguns resultados para os casos em que $k$ é igual a 2 ou 3 e os grafos $L_{i}$ são todos circuitos ou todos caminhos.

O caso em que temos apenas duas cores e os grafos $L_{1}, L_{2}$ são caminhos foi resolvido completamente há algum tempo em [15]. Se $P_{n}$ denota um caminho com $n$ vértices, sabe-se que, para $n \geq m \geq 2$,

$$
R\left(P_{n}, P_{m}\right)=n+\left\lfloor\frac{m}{2}\right\rfloor-1 .
$$

Em particular, temos que $R\left(P_{n}, P_{n}\right)=\left\lfloor\frac{3 n-2}{2}\right\rfloor$.

De modo semelhante, o caso em que os grafos $L_{1}, L_{2}$ são circuitos foi completamente solucionado por Bondy e Erdôs em [4]. Recentemente, Károlyi e Rosta conseguiram refazer este trabalho de forma simples e autocontida em [20]. Este resultado pode ser resumido no teorema a seguir, onde $C_{n}$ denota um circuito de tamanho $n$.

Teorema 1.1. Sejam $3 \leq m \leq n$ inteiros. Então

$$
R\left(C_{n}, C_{m}\right)= \begin{cases}6, & m=n=3,4 \\ n+m / 2-1, & m, n>4, \text { são pares } \\ \max \{2 m-1, n+m / 2-1\}, & m \text { é par e } n \text { é ímpar } \\ 2 n-1, & \text { caso contrario }\end{cases}
$$

Por outro lado, calcular o valor do número de Ramsey $R\left(C_{n}, C_{m}, C_{k}\right)$, onde $n, m$ e $k$ são naturais quaisquer, ainda é um problema em aberto. Contudo, existem alguns 
resultados sobre situações particulares. Vejamos alguns resultados recentes. Bondy e Erdős, ainda em [4], conjecturaram que, se $n>3$ é ímpar, então

$$
R\left(C_{n}, C_{n}, C_{n}\right)=4 n-3 .
$$

Durante um bom tempo, houve poucos avanços na direção de provar tal conjectura. Até que, em 1999, Łuczak [23] provou que se $n$ é ímpar então

$$
R\left(C_{n}, C_{n}, C_{n}\right)=4 n+o(n),
$$

quando $n \rightarrow \infty$. Recentemente, Kohayakawa, Simonovits e Skokan [19] conseguiram melhorar o teorema acima. Eles provaram o teorema a seguir.

Teorema 1.2. Existe um inteiro $n_{0}$ tal que para todo $n>n_{0}$, $n$ ímpar, temos

$$
R\left(C_{n}, C_{n}, C_{n}\right)=4 n-3 .
$$

Contudo, o caso em que $n$ é par é bastante diferente do caso em que $n$ é ímpar. Recentemente, Figaj e Łuczak [10] obtiveram um resultado assintótico para este caso, mesmo quando os circuitos possuem tamanhos um pouco diferentes.

Teorema 1.3. Dados $\alpha_{1}, \alpha_{2}, \alpha_{3}>0$, temos

$$
R\left(C_{2\left\lfloor\alpha_{1} n\right\rfloor}, C_{2\left\lfloor\alpha_{2} n\right\rfloor}, C_{2\left\lfloor\alpha_{3} n\right\rfloor}\right)=\left(\alpha_{1}+\alpha_{2}+\alpha_{3}+\max \left\{\alpha_{1}, \alpha_{2}, \alpha_{3}\right\}+o(1)\right) n,
$$

quando $n \rightarrow \infty$. Isto implica que, em particular, para $n$ par temos

$$
R\left(C_{n}, C_{n}, C_{n}\right)=2 n+o(n), \quad \text { quando } n \rightarrow \infty .
$$

Pouco depois, independentemente, Gyárfás, Ruszinkó, Sárközy e Szemerédi [17] provaram um resultado semelhante, porém mais preciso, para caminhos, confirmando uma conjectura de Faudree e Schelp [9].

Teorema 1.4. Existe um inteiro $n_{0}$ tal que, para todo $n>n_{0}$, temos

$$
R\left(P_{n}, P_{n}, P_{n}\right)= \begin{cases}2 n-1, & n \text { ímpar } \\ 2 n-2, & n \text { par. }\end{cases}
$$

Durante este trabalho de mestrado, em conjunto com Jozef Skokan [2], conseguimos provar que o resultado justo,

$$
R\left(C_{n}, C_{n}, C_{n}\right)=2 n,
$$

vale quando $n$ é um inteiro par suficientemente grande. Isto será enunciado como o teorema 5.1, provado em detalhes no capítulo 5 e será o foco principal desta dissertação. 
A prova faz uso do lema da regularidade de Szemerédi e de um lema de estabilidade de [17] sobre emparelhamentos grandes em 3-colorações de grafos quase completos. Ao estudarmos a prova desse lema de estabilidade, achamos um pequeno deslize na análise de um dos subcasos dessa prova. Esse deslize acabou nos mostrando que havia um erro no próprio enunciado do lema. Contudo, esse erro foi facilmente corrigido, deixando o enunciado do lema um pouco mais fraco do que o original, e mesmo com esta versão mais fraca é possível provar o teorema principal de [17] e o nosso resultado para circuitos.

No capítulo 2, fazemos um breve resumo histórico sobre teoria de Ramsey em geral. Apresentamos então diversos conceitos básicos e fixamos alguma notação. Em seguida, introduzimos o lema da regularidade e ilustramos uma aplicação deste lema em um problema em que temos interesse. No capítulo 4, exibimos uma prova detalhada do lema de estabilidade mencionado acima. A motivação para tal capítulo é termos uma prova autocontida do nosso resultado original, o teorema 5.1. Contudo, o leitor ansioso em ver a prova deste teorema só precisa entender o enunciado do lema de estabilidade 4.1, tendo em vista que a prova deste último é muito técnica. 


\section{Capítulo 2}

\section{Conceitos básicos e breve histórico}

\subsection{Breve histórico da teoria de Ramsey}

Os teoremas do tipo Ramsey têm sido extensivamente estudados durante a última década. Eles enquadram-se na classe de problemas que chamamos de extremais. Neste tipo de problema, normalmente procuram-se funções limiares para o tamanho de certas estruturas de modo que sempre seja possível encontrar nestas uma dada subestrutura. De certa forma, teoremas do tipo Ramsey afirmam que não existe o caos absoluto dentro de tais estruturas gerais, o que torna o assunto bastante motivador. Façamos um breve resumo histórico.

Em 1928, o jovem matemático britânico Frank Plumpton Ramsey escreveu um artigo intitulado "On a problem in formal logic", publicado no "Proceedings of the London Mathematical Society" em 1930. Este artigo tratava de um problema algorítmico em lógica proposicional. Nele, Ramsey prova um resultado puramente matemático, hoje conhecido como teorema de Ramsey. Tal teorema, que a princípio era apenas mais uma ferramenta no artigo, acabou mostrando ter maior relevância do que o problema original desse artigo.

Definição 1. Uma coloração com $r$ cores, ou simplesmente uma $r$-coloração, de um conjunto $S$ é uma função $f: S \rightarrow\{1, \ldots, r\}$ qualquer. Em geral, ao exibirmos uma coloração, especificamos uma família de subconjuntos de $S,\left\{S_{1}, \ldots, S_{r}\right\}$, tal que $S_{i}=$ $\{s \in S: f(s)=i\}$ é o conjunto dos vértices que receberam a cor $i$.

Teorema 2.1 (Ramsey). Seja $G$ um conjunto infinito e sejam $k$ e $r$ inteiros positivos. Então, para toda coloração da família dos subconjuntos com $k$ elementos de $G$ com $r$ cores existe um subconjunto infinito monocromático $F$ de $G$, ou seja, tal que todos os subconjuntos de $F$ com $k$ elementos têm a mesma cor.

Uma outra versão do teorema acima é a seguinte. 
Teorema 2.2 (Ramsey, versão finita). Dados inteiros $k, r$ e $n$, existe um inteiro $N_{0}$ tal que para todo $N \geq N_{0}$ e todo conjunto $G$ com $N$ elementos temos que: para toda coloração da família dos subconjuntos com $k$ elementos de $G$ com $r$ cores existe um subconjunto monocromático $F$ de $G$, ou seja, tal que todos os subconjuntos de $F$ com $k$ elementos têm a mesma cor, tal que $|F|=n$.

Definição 2. Dados $k, r$ e $n$, denotamos o menor inteiro $N_{0}$ com a propriedade acima por $R_{r}(n ; k)$.

Observação. Às vezes, estamos interessados no caso assimétrico onde para cada cor $i$ é dado um inteiro $n_{i}$ especificando o tamanho do conjunto monocromático que queremos encontrar na cor $i$. Por exemplo, para duas cores, dados inteiros $n_{1}, n_{2}$ e $k$, existe um inteiro, que tomamos menor possível, $R_{2}\left(n_{1}, n_{2} ; k\right)$ tal que, se $|G| \geq R_{2}\left(n_{1}, n_{2} ; k\right)$, então, em toda coloração da familia dos subconjuntos com $k$ elementos de $G$ com duas cores, existe um conjunto monocromático $F_{i}$ de cor $i$ tal que $\left|F_{i}\right|=n_{i}$ para $i$ igual a 1 ou 2. Isso segue diretamente do teorema anterior já que $R_{2}\left(n_{1}, n_{2} ; k\right) \leq$ $R_{2}(n ; k)$ onde $n=\max \left\{n_{1}, n_{2}\right\}$. Para simplificar a notação denotamos $R\left(n_{1}, n_{2} ; k\right):=$ $R_{2}\left(n_{1}, n_{2} ; k\right)$

Pouco antes de Ramsey enunciar seu teorema sobre colorações de conjuntos, Issai Schur, aluno de Frobenius e influenciado bastante por este, estudava colorações de números naturais. Um dos resultados obtidos por Schur é o teorema a seguir.

Definição 3. Denotamos o conjunto dos $n$ primeiros inteiros positivos, $\{1,2, \ldots, n\}$, por $[n]$.

Teorema 2.3 (Schur). Para todo inteiro positivo $r$, existe um inteiro $n=S(r)$ tal que, para toda coloração $f:[n] \rightarrow[r]$, dos $n$ primeiros inteiros positivos com $r$ cores, existem $x, y \in[n]$ tais que $f(x)=f(y)=f(x+y)$, ou seja, $x, y$ e $x+y$ possuem a mesma cor.

Tal problema apareceu em uma tentativa de Schur de dar uma prova elegante para a versão modular da conjectura de Fermat: ele acabou concluindo que a equação $x^{m}+y^{m} \equiv z^{m}(\bmod p)$ possui solução inteira para todo $m$ e todo primo $p$ suficientemente grande. Ele tinha interesses bastante variados na matemática. Ao tentar resolver um problema sobre a distribuição de resíduos quadráticos, deparou-se com a seguinte questão: será que para todo inteiro $k$ existe um $n$ tal que toda coloração de $[n]$ com duas cores possui uma progressão aritmética monocromática de tamanho $k$ ? Schur conjecturou que sim, mas não conseguiu resolver o problema. Após algum tempo em aberto, o problema chegou aos ouvidos de Bartel Leendert van der Waerden e outros estudantes em Göttingen. Em 1927, van der Waerden deu uma resposta afirmativa para a questão, confirmando a conjectura de Schur.

Teorema 2.4 (van der Waerden). Sejam $k$ e $r$ inteiros positivos. Então existe um inteiro positivo $n=W(k, r)$ tal que para toda $r$-coloração de $[n]$ existe uma progressão aritmética monocromática de $k$ termos. 
Para instigar a curiosidade do leitor e mostrar como o teorema de Ramsey pode ser usado nas mais diversas áreas de matemática, exibimos aqui uma prova do teorema de Erdős-Szekeres (1935).

Teorema 2.5 (Erdős-Szekeres). Dado um inteiro $n$, existe um inteiro $f(n)$ tal que todo conjunto de pelo menos $f(n)$ pontos no plano, sem três pontos colineares, contém um subconjunto de tamanho $n$ que forma um n-ágono convexo.

Prova. Afirmamos que é suficiente tomar $f(n)=R(n, 5 ; 4)$. Tome um conjunto de $f(n)$ pontos no plano onde não há três pontos colineares. Temos que mostrar que existem $n$ deles que formam um $n$-ágono convexo. Vamos colorir cada conjunto de quatro desses pontos com uma de duas cores: usamos a cor 1 se esses quatro pontos formam, em alguma ordem, um quadrilátero convexo, caso contrário, usamos a cor 2. Pela escolha de $f(n)$, o teorema de Ramsey nos diz que:

1. ou existem $n$ desses pontos tais que quaisquer 4 deles formam um quadrilátero convexo;

2. ou existem 5 desses pontos tais que quaisquer 4 deles não formam um quadrilátero convexo.

Suponha que o primeiro caso acontece e considere os tais $n$ pontos. Afirmamos que estes pontos formam um polígono convexo. Se essa afirmação fosse falsa, então a envoltória convexa dos $n$ pontos consistiria de $t$ pontos, para algum $t<n$. Os pontos restantes estariam dentro do $t$-ágono determinado pela envoltória convexa destes $t$. Chamamos esses pontos de pontos internos. Agora, se fizermos uma triangularização do $t$-ágono então, como não há três pontos colineares, um dos pontos internos, digamos $x$, cairá dentro de um dos triângulos. O ponto $x$ juntamente com os vértices desse triângulo não formam um quadrilátero convexo. Isso é uma contradição. Logo, os pontos formam o $n$-ágono convexo desejado.

Suponha agora que o segundo caso acontece e considere os tais 5 pontos. Como quaisquer 4 deles formam um quadrilátero côncavo e não há 3 deles colineares, a envoltória convexa desses 5 pontos deve ser um triângulo. Logo, 2 pontos estão no interior desse triângulo. A reta que passa por eles divide o plano em dois semi-planos, um dos quais deve conter exatamente dois vértices da envoltória convexa. Esses dois vértices juntamente com os dois pontos internos formam um quadrilátero convexo. Mas isto contradiz a escolha dos 5 pontos. Logo, na verdade, este segundo caso não pode acontecer.

Nas últimas duas décadas, teoria de Ramsey deixou de ser um emaranhado de problemas e teoremas e tornou-se uma subdisciplina coesa da análise combinatória. É possível até encontrar livros específicos sobre o assunto, por exemplo, [16]. Contudo, ainda hoje, muitos são os problemas em aberto na área, como veremos nas seções seguintes. 


\subsection{Colorindo arestas de grafos}

Neste trabalho estudamos apenas colorações da família dos subconjuntos de dois elementos de $[n]=\{1,2, \ldots, n\}$. Note que isso equivale a estudar colorações das arestas de um grafo completo com $n$ vértices.

Sempre que falarmos de coloração de um grafo $G$ estaremos nos referindo a uma coloração das arestas de $G$. Escrevemos ordem para nos referirmos ao número de vértices de um grafo e tamanho para nos referirmos ao número de arestas. Uma clique é um subgrafo completo de um grafo. Ela é monocromática se foi atribuída a mesma cor para todas as suas arestas. Uma notação bastante comum em teoria de Ramsey é a seguinte.

Definição 4. Escrevemos

$$
n \rightarrow(l)_{r}
$$

se para toda $r$-coloração do grafo completo com $n$ vértices podemos encontrar uma clique monocromática de ordem $l$. Mais geralmente, escrevemos $n \rightarrow\left(l_{1}, \ldots, l_{r}\right)$ se, para qualquer $r$-coloração do grafo completo com $n$ vértices, podemos encontrar uma clique monocromática de ordem $l_{i}$ da cor $i$ para algum $1 \leq i \leq r$.

Definição 5. A função de Ramsey $R\left(l_{1}, \ldots, l_{r}\right): \mathbb{N}^{r} \rightarrow \mathbb{N}$ denota o menor inteiro $n$ tal que

$$
n \rightarrow\left(l_{1}, \ldots, l_{r}\right)
$$

Ademais, dados $l_{1}, \ldots, l_{r}$, dizemos que $R\left(l_{1}, \ldots, l_{r}\right)$ é o número de Ramsey de $l_{1}, \ldots, l_{r}$.

Usamos também $n \rightarrow(l)$ para denotar a mesma coisa que $n \rightarrow(l, l)$ e $n \rightarrow(l)_{2}$. Do mesmo modo $R(l)$ é usado para denotar $R(l, l)$. É importante observar que o papel das cores no grafo não é simétrico. Por exemplo, suponha que queremos provar que $R\left(l_{1}, l_{2}\right)=n$, para certos inteiros $l_{1}<l_{2}$ e $n$. Em particular, temos que provar que dada uma 2-coloração do grafo completo com $n$ vértices, com as cores 1 e 2 , conseguimos achar uma clique de ordem $l_{1}$ da cor 1 ou uma clique monocromática de ordem $l_{2}$ da cor 2. Contudo, se acharmos uma clique monocromática de ordem $l_{1}$ na cor 2 ainda não resolvemos o problema. Por outro lado, é fácil verificar que $R\left(l_{1}, l_{2}\right)=R\left(l_{2}, l_{1}\right)$.

Como ilustração, vamos exibir uma prova de uma versão do teorema de Ramsey, a saber a versão para coloração de arestas de um grafo completo. As provas das outras versões que enunciamos são semelhantes a esta.

Teorema 2.6 (Ramsey). A função $R$ de Ramsey está bem definida, isto é, para toda $r$-upla $\left(l_{1}, \ldots, l_{r}\right) \in \mathbb{N}^{r}$ existe $n$ tal que

$$
n \rightarrow\left(l_{1}, \ldots, l_{r}\right) .
$$


Prova. Façamos primeiro o caso em que $r=2$. Para tanto, faremos indução sobre $l_{1}+l_{2}$. Note que podemos assumir que $R(1, l)=R(l, 1)=1$ para todo $l$, por vacuidade. Ademais, é trivial verificar que $R(l, 2)=R(2, l)=l$ para todo $l$. Isto é mais do que suficiente para a base da indução. Agora tome $l_{1}, l_{2}>1$ e suponha, como hipótese de indução, que $R\left(l_{1}, l_{2}-1\right)$ e $R\left(l_{1}-1, l_{2}\right)$ existem. Afirmamos que

$$
R\left(l_{1}, l_{2}-1\right)+R\left(l_{1}-1, l_{2}\right) \rightarrow\left(l_{1}, l_{2}\right) .
$$

Seja $G$ o grafo completo com $n$ vértices, $n=R\left(l_{1}, l_{2}-1\right)+R\left(l_{1}-1, l_{2}\right)$. Fixe uma 2-coloração $\chi$ de $\mathrm{G}$. Escolha um vértice $x \in V(G)$ qualquer e defina

$$
\begin{gathered}
V_{1}=\{y \in V(G): \chi(x, y)=1\}, \\
V_{2}=\{y \in V(G): \chi(x, y)=2\}=V(G)-V_{1}-\{x\} .
\end{gathered}
$$

Então $\left|V_{1}\right|+\left|V_{2}\right|=n-1$, de modo que

1. $\left|V_{1}\right| \geq R\left(l_{1}-1, l_{2}\right)$ ou

2. $\left|V_{2}\right| \geq R\left(l_{1}, l_{2}-1\right)$.

Suponha que (1) ocorre. Pela definição de $R$, o conjunto $V_{1}$ possui uma clique monocromática da cor 2 de ordem $l_{2}$ ou possui uma clique monocromática da cor 1 de ordem $l_{1}-1$. No primeiro caso, já encontramos o que queríamos. No segundo caso, podemos acrescentar $x$ à clique de ordem $l_{1}-1$ e obter uma clique de ordem $l_{1}$ da cor 1 donde também encontramos o que queríamos. O caso em que (2) ocorre é análogo.

Finalmente, o caso em que $r>2$ também é tratado de maneira análoga. Basta fazermos a indução sobre $l_{1}+\ldots+l_{r}$ após verificar a base e que

$$
2+\sum_{i=1}^{r}\left(R\left(l_{1}, \ldots, l_{i-1}, l_{i}-1, l_{i+1}, \ldots, l_{r}\right)-1\right) \rightarrow\left(l_{1}, \ldots, l_{r}\right) .
$$

A partir desta prova concluímos também que

$$
R(l, k) \leq R(l, k-1)+R(l-1, k) .
$$

E a partir desta equação e do fato de que $R(1, l)=R(l, 1)=1=\left(\begin{array}{c}l-1 \\ 0\end{array}\right)$ é fácil provar por indução que

$$
R(l, k) \leq\left(\begin{array}{c}
k+l-2 \\
k-1
\end{array}\right) .
$$

Em particular, no caso em que $l=k$, temos

$$
R(l) \leq\left(\begin{array}{c}
2 l-2 \\
l-1
\end{array}\right)
$$


Teorema 2.7. Vale que $2^{l / 2} \leq R(l) \leq 2^{2 l-2}$.

Prova. O lado direito da inequação é uma conseqüência da observação acima, já que

$$
\left(\begin{array}{c}
2 l-2 \\
l-1
\end{array}\right) \leq \frac{2^{2 l-2}}{\sqrt{l}} \leq 2^{2 l-2}
$$

Para a segunda parte, usamos o método probabilístico de maneira bastante simples. Considere um grafo completo $G$ com $n$ vértices e pinte cada aresta de $G$ com uma entre duas cores com probabilidade igual a $1 / 2$ para cada cor e independente para cada aresta. Para $W \subset G,|W|=l$, considere a variável aleatória indicadora $X_{W}$ tal que $X_{W}=1$ se $G[W]$ é monocromático e $X_{W}=0$ caso contrário. Seja também $X=\sum_{W} X_{W}$.

Se $n<2^{l / 2}$ temos,

$$
\begin{aligned}
& \mathbb{E}(X)= \\
& =\mathbb{E}\left(\sum_{W} X_{W}\right)=\sum_{W} \mathbb{E}\left(X_{W}\right)=\sum_{W} \mathbb{P}\left(X_{W}=1\right) \\
& =\sum_{W} 2\left(\frac{1}{2}\right)^{\left(\begin{array}{l}
l \\
2
\end{array}\right)}=\left(\begin{array}{c}
n \\
l
\end{array}\right) 2^{1-\left(\begin{array}{l}
l \\
2
\end{array}\right)} \leq \frac{n^{l}}{l !} \cdot 2^{1+\frac{l}{2}} \cdot \frac{1}{2^{l^{2} / 2}} \\
& \quad \leq\left(2^{l / 2}\right)^{l} \cdot \frac{2^{1+\frac{l}{2}}}{l !} \cdot \frac{1}{2^{l^{2} / 2}}=\frac{2^{1+\frac{l}{2}}}{l !}<1 .
\end{aligned}
$$

Logo, existe uma coloração do grafo completo com $n$ vértices, $n=\left\lfloor 2^{l / 2}\right\rfloor$, que não possui clique monocromática de ordem $l$.

Como conseqüência do teorema acima temos que

$$
\sqrt{2} \leq \liminf R(s)^{1 / s} \leq \lim \sup R(s)^{1 / s} \leq 4 .
$$

Calcular o valor de $\lim R(s)^{1 / s}$ é um problema em aberto. Na verdade nem sequer sabemos se este limite existe. Entre os problemas que envolvem o comportamento assintótico da função de Ramsey este é um dos mais célebres.

Sobre o problema de calcular o valor exato de $R(s)$, ainda que para valores pequenos de $s$, sugerimos a ótima resenha [24]. Encerramos esta seção com um texto de Paul Erdős que expressa o quão difícil é computacionalmente encontrar estes valores exatos:

Suponha que um espírito maligno nos diga: "A menos que vocês descubram o valor de $R(5)$, eu exterminarei a raça humana". Nossa melhor estratégia seria talvez fazer com que todos os computadores do mundo e cientistas da computação trabalharem juntos nisto. Por outro lado, se ele perguntasse pelo valor de $R(6)$ então nossa melhor estratégia seria tentar destruí-lo antes que ele nos destruísse. 


\subsection{Teorema de Ramsey para grafos}

Uma generalização natural dos números de Ramsey é a seguinte.

Definição 6. Dados $r$ grafos $G_{1}, \ldots, G_{r}$, definimos o número de Ramsey generalizado $R\left(G_{1}, \ldots, G_{r}\right)$ como o menor inteiro $N$ tal que se as arestas do grafo completo com $N$ vértices são coloridas com $r$ cores, existe um subgrafo monocromático da cor $i$ isomorfo a $G_{i}$ para algum $1 \leq i \leq r$. Para simplificar a notação também definimos, para um grafo $G, R(G):=R(G, G)$.

Veja que estamos interessados em encontrar o grafo $G_{i}$ simplesmente como subgrafo do grafo induzido pelas arestas da cor $i$, mas não exigimos que $G_{i}$ seja um subgrafo induzido deste. Assim, a existência de $R\left(G_{1}, \ldots, G_{r}\right)$ é uma conseqüência imediata do teorema de Ramsey original. Este, por sua vez, é equivalente a tomar cada $G_{i}$ como um grafo completo. O leitor que estiver curioso em saber mais sobre o caso induzido pode consultar, por exemplo, a seção 5.3 de [16].

É trivial verificar então que $R\left(G_{1}, \ldots, G_{r}\right) \leq R\left(\left|G_{1}\right|, \ldots,\left|G_{r}\right|\right)$, onde $\left|G_{i}\right|$ é a ordem de $G_{i}$. O interessante é que para certos grafos $G_{i}$ o número à esquerda é muito menor do que o número à direita. De fato, vários dos teoremas que veremos à frente mostram que $R\left(G_{1}, \ldots, G_{r}\right)$ é da ordem de uma função linear para algumas classes de grafos enquanto que $R\left(l_{1}, \ldots, l_{r}\right)$ é pelo menos uma exponencial em $\min \left\{l_{1}, \ldots, l_{r}\right\}$, como é fácil concluir a partir do teorema 2.7 .

Notação 7. Em geral, quando não estamos enumerando uma seqüência de grafos, usamos o índice subscrito $n$ para indicar que a ordem de um grafo $G_{n}$ é $n$. Ademais faremos as seguintes convenções: $C_{n}$ é um circuito, $P_{n}$ é um caminho, e $K_{n}$ é um grafo completo, todos com $n$ vértices. Assim os tamanhos de $C_{n}$ e $P_{n}$ são $n$ e $n-1$ respectivamente. Além disso, com certo abuso de notação, usaremos $T_{n}$ sempre que quisermos falar sobre uma árvore genérica de ordem $n$. O tamanho de $T_{n}$ é $n-1$.

Para encerrar esta seção mostraremos alguns resultados simples em teoria de Ramsey para grafos.

Um fato interessante, mas de fácil verificação, é que

$$
R(G, H) \geq(\chi(G)-1)(c(H)-1)+1,
$$

onde $\chi(G)$ é o número cromático de $G$ e $c(H)$ é a ordem da maior componente conexa de $H$. Para ver isto basta exibir uma 2-coloração de um grafo completo de ordem $(c(H)-1)(\chi(G)-1)$ que não possui uma cópia de $G$ na cor 1 nem uma cópia de $H$ na cor 2. Para tanto, basta particionar os vértices de $K_{(c(H)-1)(\chi(G)-1)}$ em $(\chi(G)-1)$ conjuntos $V_{1}, \ldots, V_{\chi(G)-1}$ de ordem $(c(H)-1)$. E então pintar todas as arestas com as duas extremidades em conjuntos distintos da partição com a cor 1 e as demais arestas com a cor 2 . 
Uma aplicação desse resultado é o teorema a seguir.

Teorema 2.8. Para todos $s, t \geq 2$ temos $R\left(K_{s}, T_{t}\right)=(s-1)(t-1)+1$.

Prova. O fato de que $R\left(K_{s}, T_{t}\right) \geq(s-1)(t-1)+1$ é uma aplicação direta do fato anterior. Para provar a desigualdade contrária, considere uma 2-coloração de um grafo completo com $n=(s-1)(t-1)+1$ vértices. Seja $G^{1}$ o subgrafo induzido pelas arestas da cor 1 e $G^{2}$ o subgrafo induzido pelas arestas da cor $2\left(G^{2}\right.$ é o complemento de $\left.G^{1}\right)$. Suponha que $G^{1}$ não contém $K_{s}$ como subgrafo. Então um conjunto independente de $G^{2}$ tem ordem no máximo $s-1$. Logo, $\chi\left(G^{2}\right) \geq\lceil n /(s-1)\rceil=t$. Afirmamos que $G^{2}$ possui um subgrafo $H^{2}$ de grau mínimo pelo menos $t-1$. Admitindo isto, é fácil ver que $H^{2}$ contém uma cópia de $T_{t}$. De fato, podemos assumir indutivamente que achamos $T_{t-1} \subset H^{2}$, onde $T_{t-1}=T_{t}-x$ e $x$ é uma folha qualquer de $T_{t}$. Seja $y$ o vizinho de $x$ em $T_{t}$. Como $y$ tem pelo menos $t-1$ vizinhos em $H^{2}$, pelo menos um desses vizinhos, digamos $z$, não pertence a $T_{t-1}$. Logo, o subgrafo de $H^{2}$ induzido por $T_{t-1} \cup z$ claramente contém uma cópia de $T_{t}$.

Resta provar a existência de $H^{2}$. Suponha o contrário, ou seja, que $G^{2}$ não possui um subgrafo de grau mínimo pelo menos $t-1$. Então todo subgrafo $H$ de $G^{2}$ tem grau mínimo no máximo $t-2$, ou seja, possui um vértice de grau menor ou igual a $t-2$. Em particular, o grafo $H_{n}=G^{2}$ tem um vértice $x_{n}$ de grau no máximo $t-2$. Seja $H_{n-1}=H_{n} \backslash\left\{x_{n}\right\}$. Novamente $H_{n-1}$ possui um vértice $x_{n-1}$ de grau no máximo $t-2$. Faça então $H_{n-2}=H_{n-1} \backslash\left\{x_{n-1}\right\}$. Continue fazendo isto até que todos os vértices de $G^{2}$ tenham sido enumerados. Agora, a seqüência $x_{1}, \ldots, x_{n}$ é tal que cada $x_{i}$ é adjacente a no máximo $t-2$ vértices anteriores a ele. Então um algoritmo guloso para colorir os vértices de $G^{2}$ usaria no máximo $t-1$ cores, uma contradição.

Notação 8. Dados dois grafos $G=(V(G), E(G))$ e $H=(V(H), E(H))$, tais que $V(G) \cap V(H)=\emptyset$, o grafo união $G \cup H$ é definido simplesmente como

$$
G \cup H=(V(G) \cup V(H), E(G) \cup E(H)) .
$$

Ademais, para um inteiro $k$, definimos $k G$ como a união de $k$ cópias disjuntas de $G$.

Outro resultado simples que também pode ter bastante utilidade é o que segue abaixo.

Teorema 2.9. Para quaisquer grafos $G, H^{1}, H^{2}$, onde $V\left(H_{1}\right) \cap V\left(H_{2}\right)=\emptyset$,

$$
R\left(G, H^{1} \cup H^{2}\right) \leq \max \left\{R\left(G, H^{1}\right)+\left|H^{2}\right|, R\left(G, H^{2}\right)\right\} .
$$

Em particular, $R(G, s H) \leq R(G, H)+(s-1)|H|$.

Prova. Seja $n=\max \left\{R\left(G, H^{1}\right)+\left|H^{2}\right|, R\left(G, H^{2}\right)\right\}$ e suponha que o grafo $K_{n}$ seja 2-colorido sem que exista um subgrafo $G$ contido no grafo induzido pelas arestas de 
cor 1. Então, como $n \geq R\left(G, H^{2}\right)$, deve existir um grafo $H^{2}$ na cor 2 . Removendo-se este grafo de $K_{n}$ ainda sobram pelo menos $R\left(G, H^{1}\right)$ vértices. Logo, o grafo resultante deve conter um $H^{1}$ da cor 2 . Assim, o $K_{n}$ contém um $H^{1} \cup H^{2}$ na cor 2 . Isto completa a prova da primeira desigualdade.

Agora, dados grafos $G$ e $H$, fazendo $H^{1}=(s-1) H$ e $H^{2}=H$, temos que

$$
R(G, s H) \leq \max \{R(G,(s-1) H)+|H|, R(G, H)\}=R(G,(s-1) H)+|H|,
$$

já que $R(G,(s-1) H) \leq R(G, H)$. Agora, é trivial provar a segunda desigualdade do enunciado usando indução.

Usando o teorema acima é possível calcular $R\left(s K_{2}, t K_{2}\right)$.

Teorema 2.10. Se $s \geq t \geq 1$ então $R\left(s K_{2}, t K_{2}\right)=2 s+t-1$.

Prova. O grafo $G_{2 s+t-2}=K_{2 s-1} \cup(t-1) K_{1}$ não contém $s$ arestas independentes, ou seja, não contém $s K_{2}$. Ademais, seu complemento não contém $t K_{2}$. Com isto, é trivial concluir que $R\left(s K_{2}, t K_{2}\right) \geq 2 s+t-1$.

É trivial verificar também que $R\left(s K_{2}, K_{2}\right)=2 s$. Assim, para completar a prova é suficiente verificar que

$$
R\left(s K_{2}, t K_{2}\right) \leq R\left((s-1) K_{2},(t-1) K_{2}\right)+3
$$

e usar indução sobre $\min \{s, t\}$. Como de costume, seja $n=R\left((s-1) K_{2},(t-1) K_{2}\right)+3$, considere uma 2-coloração de $K_{n}$ e denote por $G^{i}$ o grafo induzido pelas arestas da cor $i$, para $i=1,2$. Se $G^{1}=K_{n}$ então $s K_{2} \subset G^{1}$, e se $G^{2}=K_{n}$ então $t K_{2} \subset G^{1}$, pois $n \geq 2 s \geq 2 t$. Caso contrário, existem três vértices, digamos $x, y$ e $z$, tais que $x y \in G^{1}$, $x z \in G^{2}$. Agora, $K_{n} \backslash\{x, y, z\}$ contém ou um $(s-1) K_{2}$ da cor 1 ou um $(t-1) K_{2}$ da cor 2. No primeiro caso, adicionamos a aresta $x y$ para encontrar o nosso $s K_{2}$. No segundo, adicionamos a aresta $x z$ e encontramos o $t K_{2}$.

Finalmente, uma generalização do teorema anterior ([6]) foi usada em um trecho da prova do lema 4.1 no capítulo 4 , um dos focos principais neste trabalho, estudado no artigo [17].

Teorema 2.11 (Cockayne e Lorimer, 1975). Dados $n_{1}, \ldots, n_{t} \geq 1$ inteiros tais que $n_{1}=\max \left\{n_{1}, \ldots, n_{t}\right\}$ então

$$
R\left(n_{1} K_{2}, \ldots, n_{t} K_{2}\right)=n_{1}+1+\sum_{i=1}^{t}\left(n_{i}-1\right) .
$$

Corolário 2.12. Dado um inteiro positivo $n$ vale que

$$
R\left(n K_{2}, n K_{2}, n K_{2}\right)=4 n-2 .
$$




\subsection{Teoremas de Ramsey versus teoremas de densidade}

Em geral, um problema do tipo Ramsey pode ser formulado da seguinte maneira: dada uma quantidade $r$ de cores, queremos determinar se em toda $r$-coloração de um conjunto de objetos podemos encontrar um subconjunto monocromático desses objetos com uma dada propriedade. Esta questão pode ser formulada em termos de hipergrafos. Se $V$ é o conjunto dos objetos que iremos colorir e $E$ é a família dos subconjuntos de $V$ que satisfazem uma certa propriedade então podemos formalizar a pergunta como: dado o hipergrafo $H=(V, E)$, é verdade que, em toda $r$-coloração de $V$, existe uma hiperaresta monocromática, ou seja, um conjunto $e \in E$ tal que os vértices pertencentes a $e \subset V$ possuem todos a mesma cor? Isso nos motiva a fazer a definição a seguir.

Definição 9. Dado um hipergrafo $H$, seu número cromático $\chi(H)$ é o menor inteiro tal que existe uma coloração dos vértices de $H$ onde nenhuma das hiperarestas de $H$ é monocromática. Veja que isto é uma generalização natural do número cromático para grafos.

Um outro tipo de problema é relacionado à próxima definição.

Definição 10. Dado um hipergrafo $H=(V, E)$, definimos a função de Turán $T(H)$ como o menor inteiro $T_{0}$ tal que para todo subconjunto $V^{\prime}$ de $V$ com pelo menos $T_{0}$ vértices existe $e \in E$ tal que $e \subset V^{\prime}$. Defina $\tau(H)=T(H) /|V(H)|$.

Notação 11. Para um conjunto $S$ e um inteiro $l$ denotamos $\left(\begin{array}{c}S \\ l\end{array}\right):=\{F \subset S ;|F|=l\}$.

Em geral, não sabemos como calcular $T(H)$. Mas o valor de $T(H)$ é conhecido para certas famílias de hipergrafos. Por exemplo, considere o hipergrafo $H_{n}=\left(V_{n}, E_{n}\right)$, onde $V_{n}=\left(\begin{array}{c}{[n]} \\ 2\end{array}\right)$ e $E_{n}=\left\{\left(\begin{array}{c}S \\ 2\end{array}\right): S \in\left(\begin{array}{c}{[n]} \\ l\end{array}\right)\right\}$, para algum $l$. Veja que calcular $T\left(H_{n}\right)$ é equivalente a encontrar o menor número de arestas $t$ tal que todo grafo com $n$ vértices e pelo menos $t$ arestas possui uma clique de ordem $l$. Um importante teorema de densidade é o teorema de Turán [30] que nos dá o valor exato de $T\left(H_{n}\right)$. Veja também o livro de Bollobás [3].

Teorema 2.13. Dados $n$ e $l$, o menor inteiro $t$ tal que todo grafo com $n$ vértices e $t$ arestas possui uma clique de ordem $l$ é dado por

$$
t=t(n, l)=1+\left(\begin{array}{l}
n \\
2
\end{array}\right)-k\left(\begin{array}{c}
m+1 \\
2
\end{array}\right)-(l-1-k)\left(\begin{array}{c}
m \\
2
\end{array}\right)
$$

onde $m$ e $k$ são tais que $n=(l-1) m+k$ e $0 \leq k<l-1$ (de modo que $m$ e $k$ estão unicamente determinados a partir de $n$ e l). Ademais, o único grafo (a menos de isomorfismos) com $n$ vértices e $(t-1)$ arestas que não possui uma clique de ordem $l$ é obtido particionando os $n$ vértices em $(l-1)$ conjuntos de ordem $m$ ou $m+1$ e colocando arestas entre pares de vértices que pertencem a conjuntos distintos dessa partição. 
Vamos agora fazer uma breve comparação entre propriedades do tipo Ramsey e propriedades de densidade. Dado um hipergrafo $H$ e um inteiro $r$, considere as seguintes afirmações:

$$
\begin{array}{ll}
A: & \chi(H)>r ; \\
B: & \tau(H) \leq 1 / r .
\end{array}
$$

E, para uma seqüência de hipergrafos $H_{n}=\left(V_{n}, E_{n}\right)$, considere as afirmações análogas:

$$
\begin{array}{lll}
A^{*}: & \chi\left(H_{n}\right)>r, & \text { quando } n \rightarrow \infty \\
B^{*}: & \tau\left(H_{n}\right) \leq 1 / r & \text { quando } n \rightarrow \infty
\end{array}
$$

As afirmações $A$ e $A^{*}$ são afirmações do tipo Ramsey. Enquanto que as afirmações $B$ e $B^{*}$ são afirmações de densidade. A afirmação $A$ diz que, se as hiperarestas de $H$ forem coloridas com $r$ cores, então encontraremos uma hiperaresta monocromática. A afirmação $B$ diz que, se um conjunto de vértices não é muito pequeno, então ele contém uma hiperaresta de $H$. É muito fácil verificar a validade do seguinte teorema.

Teorema 2.14. Valem as seguintes implicações: (i) $B$ implica $A$; (ii) $B^{*}$ implica $A^{*}$.

Contudo, as implicações recíprocas, " $A$ implica $B$ " e " $A *$ implica $B$ " são falsas. Considere, por exemplo, suas interpretações para teoria de Ramsey clássica (como na seção 2.2), onde o conjunto das arestas de um grafo é $r$-colorido e queremos achar uma clique monocromática de ordem $l$. Precisamente, se olharmos novamente para os hipergrafos $H_{n}=\left(V_{n}, E_{n}\right)$, onde $V_{n}=\left(\begin{array}{c}{[n]} \\ 2\end{array}\right)$ e $E_{n}=\left\{\left(\begin{array}{c}S \\ 2\end{array}\right): S \in\left(\begin{array}{c}{[n]} \\ l\end{array}\right)\right\}$, temos uma seqüência $\left(H_{n}\right)$ para a qual vale $A^{*}$ e não vale $B^{*}$.

De fato, veja que a afirmação $\chi\left(H_{n}\right)>r$ é idêntica a $n \rightarrow(l)_{r}$. Assim, pelo teorema 2.6 (Ramsey), a seqüência $\left\{H_{n}\right\}$ satisfaz $A^{*}$. Por outro lado, o teorema 2.13 nos diz que se escrevermos $n=(l-1) m+k$ com $0 \leq k<l-1$, então

$$
T\left(H_{n}\right)=1+\left(\begin{array}{l}
n \\
2
\end{array}\right)-k\left(\begin{array}{c}
m+1 \\
2
\end{array}\right)-(l-1-k)\left(\begin{array}{c}
m \\
2
\end{array}\right) .
$$

Logo,

$$
\lim _{n \rightarrow \infty} \tau\left(H_{n}\right)=1-\frac{1}{l-1} .
$$

Para $r \geq 2$ e $l \geq 4$ temos que $1-\frac{1}{l-1}>1 / r$ de modo que $B^{*}$ é falsa. Esta mesma construção nos dá exemplos de hipergrafos para os quais " $A$ implica $B$ " não vale.

Um dos mais famosos teoremas de densidade é o teorema de Szemerédi, que generaliza o teorema 2.4 (van der Waerden).

Teorema 2.15 (Szemerédi [28]). Para todo inteiro $l$ e real $\varepsilon>0$ existe um inteiro $n_{0}(\varepsilon, l)$ tal que se $n>n_{0}(\varepsilon, l)$ e $S \subseteq[n]$ tem densidade maior que $\varepsilon$, ou seja, $|S|>\varepsilon n$, então $S$ contém uma progressão aritmética de tamanho $l$. 
O teorema de van der Waerden foi provado em 1927. Em 1936, Erdős e Turán conjecturaram o teorema acima. Para $l=2$ o teorema é trivial. O caso particular em que $l=3$ foi provado em 1953 por Roth [25]. Em 1969, o caso particular em que $l=4$ foi provado por Szemerédi [27] e ele mesmo provou o teorema geral acima em 1973. A prova completa desse teorema é extremamente complexa. 


\section{Capítulo 3}

\section{Lema da regularidade de Szemerédi}

Alguns problemas em teoria extremal dos conjuntos podem ser resolvidos usando apenas técnicas de matemática elementar. Em outros faz-se necessário o uso de técnicas sofisticadas, como o lema da regularidade de Szemerédi e lemas de estabilidade. Em Teoria de Ramsey para grafos, este é o caso dos problemas nos artigos [5, 19, 23], que tratam de números de Ramsey para circuitos ou caminhos envolvendo mais de três cores, para os quais até agora não existem provas elementares. Nas seções seguintes falaremos sobre os resultados obtidos nestes artigos. Agora nos concentraremos na principal ferramenta usada nestes artigos.

O lema da regularidade tem se mostrado uma das mais poderosas ferramentas para lidar com problemas extremais. Daí a motivação em estudá-lo. Este lema foi concebido originalmente como um lema auxiliar na prova do teorema 2.15. Basicamente, ele nos diz que qualquer grafo pode ser, de certo modo, aproximado por uma união de grafos bipartidos pseudo-aleatórios. Assim, o lema da regularidade nos ajuda a carregar certos resultados, que são triviais na classe dos grafos aleatórios, para a classe de grafos em geral, onde provavelmente seria mais difícil provar que essas propriedades valem. As seguintes definições tornam preciso o que acabamos de dizer.

Definição 12. Dados um grafo $G=(V, E)$ e conjuntos $X, Y \subset V$ disjuntos, definimos $e(X, Y)=e_{G}(X, Y)=|\{x y \in E: x \in X, y \in Y\}|$. E para $X, Y$ não vazios também definimos a densidade do par $(X, Y)$ em $G$ como

$$
d(X, Y)=d_{G}(X, Y)=\frac{e(X, Y)}{|X| \cdot|Y|} .
$$

Definição 13. Seja $\varepsilon>0$. Dado um grafo $G$ e dois conjuntos de vértices disjuntos $A, B \subset V(G)$, dizemos que o par $(A, B)$ é e-regular se para todos $X \subset A$ e $Y \subset B$ satisfazendo

$$
|X|>\varepsilon|A| \quad \text { e } \quad|Y|>\varepsilon|B|,
$$


temos

$$
|d(X, Y)-d(A, B)|<\varepsilon .
$$

Definição 14. Dizemos que um par $(A, B)$ é $\varepsilon$-regular com densidade $d$ se $(A, B)$ é E-regular e $d(A, B)=d$.

Isto nos diz, essencialmente, que as arestas entre os conjuntos $A$ e $B$ se comportam como as de um grafo bipartido aleatório, onde cada aresta tem probabilidade $d(A, B)$ de pertencer a tal grafo.

Proposição 3.1. O par $(A, B)$ é $\varepsilon$-regular no grafo $G$ se e somente se é $\varepsilon$-regular em $\bar{G}$, o complemento de $G$.

Prova. Basta observar que $d_{\bar{G}}(A, B)=1-d_{G}(A, B)$, para todos $A \subset V(G), B \subset V(G)$ disjuntos. Assim, na equação (3.1) temos

$\left|d_{\bar{G}}(X, Y)-d_{\bar{G}}(A, B)\right|=\left|\left(1-d_{G}(X, Y)\right)-\left(1-d_{G}(A, B)\right)\right|=\left|d_{G}(X, Y)-d_{G}(A, B)\right|$.

Definição 15. Dado um vértice $x$ e um conjunto de vértices $Y$, definimos $\operatorname{deg}(x, Y)$ como o número de vizinhos de $x$ em $Y$, ou seja, $\operatorname{deg}(x, Y)=|\{y \in Y:(x, y) \in E(G)\}|$.

Proposição 3.2 (A maioria dos vértices possui grau alto). Seja $(A, B)$ um par $\varepsilon$-regular com densidade $d$, sobre um grafo $G$ qualquer. Dado $Y \subset B$ tal que $|Y|>\varepsilon|B|$, temos que

$$
|\{x \in A: \operatorname{deg}(x, Y) \leq(d-\varepsilon)|Y|\}| \leq \varepsilon|A| .
$$

Prova. Suponha por absurdo que existe $Y \subset B,|Y|>\varepsilon|B|$ tal que

$$
|\{x \in A: \operatorname{deg}(x, Y) \leq(d-\varepsilon)|Y|\}|>\varepsilon|A| .
$$

Seja $X=\{x \in A: \operatorname{deg}(x, Y) \leq(d-\varepsilon)|Y|\}$. Temos que

$$
e(X, Y)=\sum_{x \in X} \operatorname{deg}(x, Y) \leq(d-\varepsilon)|X| \cdot|Y|,
$$

e portanto

$$
d(X, Y) \leq d-\varepsilon,
$$

o que contraria o fato de $(A, B)$ ser $\varepsilon$-regular.

O lema a seguir sobre pares regulares, além de interessante por si só, será particularmente importante na prova do teorema principal do capítulo 5. Informalmente, ele nos diz que um grafo bipartido suficientemente grande com bipartição $V_{1} \cup V_{2}$ onde $\left(V_{1}, V_{2}\right)$ é um par $\varepsilon$-regular possui caminhos de quase todos os tamanhos possíveis entre quase todos os pares de vértices $v^{\prime} \in V_{1}, v^{\prime \prime} \in V_{2}$ (mesmo que a densidade entre $V_{1}$ e $V_{2}$ seja pequena). 
Observação. Ao definirmos constantes durante o enunciado de um teorema/lema, como no seguinte, usaremos como índice subscrito da constante o número desse teorema/lema (no lugar do tradicional índice 0). Isto facilita futuras referências a tais constantes.

Lema 3.3. Seja $G$ um grafo bipartido com bipartição $V_{1} \cup V_{2}$ tal que $\left|V_{1}\right|=\left|V_{2}\right|=n$. Suponha que $\left(V_{1}, V_{2}\right)$ é $\varepsilon$-regular com densidade pelo menos $\alpha / 4$, para algum $0<\alpha \leq 1$ e $0<\varepsilon<0.01 \alpha$. Então existe $n_{3.3}$ tal que se $n>n_{3.3}$, então para todo par de vértices $v^{\prime} \in V_{1}, v^{\prime \prime} \in V_{2}$, onde $\operatorname{deg}\left(v^{\prime}\right), \operatorname{deg}\left(v^{\prime \prime}\right) \geq \alpha n / 5$, e todo $l$, onde $1 \leq l \leq n-5 \varepsilon n / \alpha$, temos que $G$ contém um caminho de comprimento $2 l+1$ conectando $v^{\prime}$ e $v^{\prime \prime}$.

Prova. Tome $v^{\prime}$ e $v^{\prime \prime}$ como acima. Considere primeiro o caso em que $1 \leq l \leq \alpha n / 6$. Seja

$$
V_{i}^{-}=\left\{v \in V_{i}: \operatorname{deg}(v)<\alpha n / 5\right\}, \text { para } i=1,2 .
$$

Como $\alpha n / 5<(\alpha / 4-\varepsilon) n$, pela proposição $3.2,\left|V_{i}^{-}\right| \leq \varepsilon n$. Assim, se definirmos

$$
V_{i}^{+}=V_{i} \backslash V_{i}^{-},
$$

temos que $\left|V_{i}^{+}\right| \geq(1-\varepsilon) n$. Jogando fora alguns vértices de $V_{1}^{+}$e $V_{2}^{+}$, podemos obter $\hat{V}_{1} \subseteq V_{1}^{+}$e $\hat{V}_{2} \subseteq V_{2}^{+}$, tais que $\left|\hat{V}_{1}\right|=\left|\hat{V}_{2}\right| \geq(1-\varepsilon) n$ e $v^{\prime} \in \hat{V}_{1}, v^{\prime \prime} \in \hat{V}_{2}$. É fácil ver que o subgrafo $H$ induzido por $\hat{V}_{1} \cup \hat{V}_{2}$ tem grau mínimo pelo menos $\alpha n / 5-\varepsilon n$. Construa gulosamente um caminho de comprimento $2 l-2$, digamos $P_{2 l-2}=v_{0} v_{1} \ldots v_{2 l-2}$, tal que $v_{0}=v^{\prime}$ e $V\left(P_{2 l-2}\right) \subseteq \hat{V}_{1} \cup \hat{V}_{2} \backslash\left\{v^{\prime \prime}\right\}$. Ou seja, comece escolhendo $v_{0}=v^{\prime}$ e dado que foram escolhidos $v_{0}, \ldots, v_{i-1}$, escolha $v_{i}$ como qualquer vértice da vizinhança de $v_{i-1}$ em $H$ que não pertença a $\left\{v_{0}, \ldots, v_{i-1}\right\} \cup\left\{v^{\prime \prime}\right\}$. Tal vértice $v_{i}$ existe pois $l \leq \alpha n / 6$ e $\operatorname{deg}\left(v_{i-1}\right) \geq \alpha n / 5-\varepsilon n$, e portanto $\operatorname{deg}\left(v_{i-1}\right)-l \geq 1$. Para extender $P_{2 l-2}$ de modo a obter um caminho que termine em $v^{\prime \prime}$ e tenha comprimento $2 l+1$, basta mostrar que $G$ contém uma aresta $\left\{v_{2 l-1}, v_{2 l}\right\}$ de $N_{H}\left(v_{2 l-2}\right) \backslash\left(V\left(P_{2 l-2}\right) \cup\left\{v^{\prime \prime}\right\}\right)$ para $N_{H}\left(v^{\prime \prime}\right) \backslash V\left(P_{2 l-2}\right)$. Neste caso, $P_{2 l+1}=P_{2 l-2} v_{2 l-1} v_{2 l} v^{\prime \prime}=v_{0} \ldots v_{2 l} v^{\prime \prime}$ será o caminho que procuramos. De fato, tal aresta existe já que,

$$
\left|N_{H}\left(v_{2 l-2}\right) \backslash\left(V\left(P_{2 l-2}\right) \cup\left\{v^{\prime \prime}\right\}\right)\right| \geq \alpha n / 5-\varepsilon n-\alpha n / 6-1>\varepsilon n
$$

e analogamente

$$
\left|N_{H}\left(v^{\prime \prime}\right) \backslash V\left(P_{2 l-2}\right)\right|>\varepsilon n \text {. }
$$

Assim, pela $\varepsilon$-regularidade de $\left(V_{1}, V_{2}\right)$, a densidade entre esses dois conjuntos não pode ser nula.

Agora suponha que $\alpha n / 6 \leq l \leq n-5 \varepsilon n / \alpha$ e já construímos um caminho de comprimento $2 l-1$, digamos $P_{2 l-1}=v_{0} v_{1} \ldots v_{2 l-1}$, onde $v_{0}=v^{\prime}$ e $v_{2 l-1}=v^{\prime \prime}$. Neste caso a estratégia é diferente: queremos aumentar este caminho substituindo uma de suas arestas por um caminho de tamanho 3. Diremos que um vértice $v \in V\left(P_{2 l-1}\right)$ é bom se ele possui pelo menos $\varepsilon n$ vizinhos fora de $V\left(P_{2 l-1}\right)$, ou seja, $\left|N(v) \backslash V\left(P_{2 l-1}\right)\right| \geq \varepsilon n$, e diremos que ele é ruim caso contrário. 
Se existe $i$, com $0 \leq i \leq 2 l-2$, tal que $v_{i} \in V\left(P_{2 l-1}\right) \cap V_{1}$ e $v_{i+1} \in V\left(P_{2 l-1}\right) \cap V_{2}$ são bons, como no primeiro caso, novamente pela $\varepsilon$-regularidade de $\left(V_{1}, V_{2}\right)$, a densidade entre $N\left(v_{i}\right) \backslash V\left(P_{2 l-1}\right)$ e $N\left(v_{i+1}\right) \backslash V\left(P_{2 l-1}\right)$ não pode ser nula. Concluímos então que existem $w^{\prime}, w^{\prime \prime} \notin V\left(P_{2 l-1}\right)$ tais que $\left\{v_{i}, w^{\prime}\right\},\left\{w^{\prime}, w^{\prime \prime}\right\}$ e $\left\{w^{\prime \prime}, v_{i+1}\right\}$ são arestas de $G$. Consequentemente, o caminho $v_{0} v_{1} \ldots v_{i} w^{\prime} w^{\prime \prime} v_{i+1} \ldots v_{2 l-1}$ tem comprimentos $2 l+1 \mathrm{e}$ conecta $v^{\prime}$ a $v^{\prime \prime}$. Resta então provar que existe tal $i$.

Seja $Y=V_{2} \backslash V\left(P_{2 l-1}\right)$. Lembre que $|Y| \geq \frac{5}{\alpha} \varepsilon n>\varepsilon n$. E seja $X$ o conjunto dos vértices de $V_{1}$ que possuem grau menor ou igual a $(\alpha / 4-\varepsilon)|Y|$ em $Y$. Pela proposição $3.2,|X| \leq \varepsilon n$. Como

$$
(\alpha / 4-\varepsilon)|Y|>\alpha / 5|Y| \geq \varepsilon n,
$$

todos os vértices ruins de $V_{1}$ pertencem a $X$ e portanto existem no máximo $\varepsilon n$ vértices ruins em $V_{1}$. De modo análogo existem no máximo $\varepsilon n$ vértices ruins em $V_{2}$. Como existem $l$ arestas independente em $P_{2 l-1}$ e no máximo $2 \varepsilon n<\frac{\alpha}{6} \varepsilon n \leq l$ vértices ruins, então os vértices ruins não cobrem todas as arestas de $P_{2 l-1}$ e portanto existe o índice $i$ procurado.

Observação. O lema acima é uma versão ligeiramente mais forte do "claim 3" de [23]. $O$ "claim 3" de [23] não era forte o suficiente para ser usado na prova do teorema do capítulo 5. Contudo, a idéia da prova fizemos acima é basicamente a mesma da prova da versão original do lema.

A seguir, enunciaremos formalmente o Lema de Szemerédi. Não iremos exibir a prova deste lema, mas recomendamos a leitura do artigo original ([29]) e da seção 7,4 do livro de Diestel [7].

Teorema 3.4 (Lema da regularidade, Szemerédi 1978 [29]). Para todo $\varepsilon>0$ e $m$ inteiro existem inteiros $M(\varepsilon, m)$ e $N(\varepsilon, m)$ com a seguinte propriedade: para todo grafo $G$ com $n \geq N(\varepsilon, m)$ vértices existe uma partição do conjunto dos vértices em $t+1$ classes $V=V_{0} \cup V_{1} \cup \ldots \cup V_{t}$ tal que

- $m \leq t \leq M(\varepsilon, m)$;

- $\left|V_{0}\right|<\varepsilon n$;

- $\left|V_{1}\right|=\left|V_{2}\right|=\ldots=\left|V_{t}\right|$;

- todos exceto no máximo $\varepsilon t^{2}$ dos pares $\left(V_{i}, V_{j}\right)$ com $i, j>0$ e $i \neq j$ são $\varepsilon$-regulares.

Observação. A existência da classe $V_{0}$ é puramente técnica: ela permite que seja possível que as demais classes tenham o mesmo número de elementos. Poderíamos supor que $V_{0}=\emptyset$ se relaxassemos a condição $\left|V_{i}\right|=\left|V_{j}\right|$ para ||$V_{i}|-| V_{j}|| \leq 1$.

Isto quer dizer que o conjunto de vértices de todo grafo suficientemente grande pode ser particionado em um número limitado de conjuntos, de modo que, para quase todo 
par desses conjuntos vale que as arestas entre eles induzem um grafo bipartido que é pseudo-aleatório. Observe que o lema é interessante quando o grafo em questão tem densidade positiva de arestas. Caso contrário, ele pode ser trivialmente aproximado pela união de grafos bipartidos vazios.

Definição 16. Dado um grafo $G=(V, E)$, uma partição $P$ do conjunto de vértices $V$ em $V_{1}, \ldots, V_{t}$, e dois parâmetros $\varepsilon$, $d$, definimos o grafo reduzido $R=R(d, \varepsilon)$ como segue: os vértices de $R$ são os conjuntos $V_{1}, \ldots, V_{t}$ e $V_{i}$ é adjacente a $V_{j}$ se e só se $\left(V_{i}, V_{j}\right)$ é $\varepsilon$-regular com densidade maior ou igual a $d$.

A maioria das aplicações do lema da regularidade usa o grafo reduzido e depende do fato de que muitas propriedades deste grafo são herdadas por $G$. Por exemplo, se estamos interessados em achar subgrafos do grafo original $G$, então a proposição 3.5 a seguir é bastante interessante.

Precisamos primeiro da seguinte definição. Dado um grafo $R$ o grafo $R^{s}$ é obtido através da substituição de cada vértice $v$ de $R$ por um conjuntos de $s$ vértices e cada aresta de $R$ por um grafo bipartido completo entre os conjuntos de $s$ vértices correspondentes.

Proposição 3.5. Para todo $d \in(0 ; 1], \Delta>1$ e $s \geq 1$ existe $\varepsilon_{0}>0$ e $n_{0}$ com a seguinte propriedade. Dado um grafo $G_{n}$ tal que $n \geq n_{0}$, um grafo $H$ tal que $\Delta(H) \leq \Delta$ e $R$ um grafo reduzido de $G_{n}$ com parâmetros $\varepsilon \leq \varepsilon_{0}$ ed temos que se $H$ é subgrafo de $R^{s}$ então $H$ é subgrafo de $G_{n}$.

Em problemas de coloração, é comum trabalharmos com os subgrafos induzidos pelas arestas de cada uma das cores. É possível enunciar o lema da regularidade de modo que seja possível encontrar uma partição que satisfaça as condições do lema para todos esses subgrafos simultaneamente. Não é difícil adaptar a prova original do teorema 3.4 de modo a obtermos o teorema seguinte.

Teorema 3.6 (Lema da regularidade para várias cores). Para todo $\varepsilon>0$, $m$ e $s$ inteiros existem inteiros $M_{3.6}=M(\varepsilon, m, s)$ e $N_{3.6}=N(\varepsilon, m, s)$ com a seguinte propriedade: para todos os grafos $G_{1}, G_{2}, \ldots, G_{s}$ sobre o mesmo conjunto de vértices $V$ de ordem $n \geq N_{3.6}$ existe uma partição de $V$ em $t+1$ classes $V=V_{0} \cup V_{1} \cup \ldots \cup V_{t}$ tal que

- $m \leq t \leq M_{3.6}$;

- $\left|V_{0}\right|<\varepsilon n$;

- $\left|V_{1}\right|=\left|V_{2}\right|=\ldots=\left|V_{t}\right|$

- todos exceto no máximo $\varepsilon t^{2}$ dos pares $\left(V_{i}, V_{j}\right)$, com $i, j>0, i \neq j$, são $\varepsilon$-regulares em relação a cada $G_{k}, 1 \leq k \leq s$. 


\subsection{Uma aplicação do lema da regularidade}

Para ilustrar como o lema de regularidade (teorema 3.4) pode ser utilizado, exibiremos a prova do seguinte teorema retirado do artigo [5].

Teorema 3.7. Para cada inteiro positivo d, existe uma constante $c$, tal que se $G$ é um grafo com $n$ vértices com grau máximo menor ou igual a $d$ então o número de Ramsey do grafo $G, R(G)=R(G, G)$, é menor ou igual a $c n$.

Prova. Seja $d$ um inteiro positivo qualquer. Escolha um inteiro $m$ (grande) tal que

$$
\frac{1}{2} \log (m / 3) \geq d+1
$$

Então, escolha $\varepsilon=1 / m$. Observe que, com isso, também temos que $1 / 3^{d}>2 d \varepsilon$. De fato

$$
m=\frac{1}{\varepsilon} \geq 3 \exp (2(d+1)) \Rightarrow 2 d \varepsilon \leq \frac{2 d}{3 \exp (2(d+1))}<\frac{1}{3^{d}} .
$$

Sejam $M=M(\varepsilon, m)$ e $N=N(\varepsilon, m)$ as constantes obtidas ao aplicarmos o lema da regularidade, ou seja, o teorema 3.4. Afirmamos que $c=\max \{N, M / d \varepsilon\}$ é a constante procurada. Note que, de fato, $c$ é determinada apenas em função de $d$.

Seja então $G$ um grafo com $n$ vértices e grau máximo menor ou igual a $d$. Mostremos que $R(G) \leq c n$. Considere uma coloração arbitrária de $K_{c n}$, o grafo completo com $c n$ vértices, usando duas cores, digamos azul e vermelha. Seja $H$ o grafo sobre $c n$ vértices determinado pelas arestas vermelhas, e $\tilde{H}$ o grafo determinado pelas arestas azuis.

Como $H$ tem $c n$ vértices e $c n>N$, podemos aplicar o lema da regularidade, teotema 3.4, e a observação que segue tal lema, ao grafo $H$ e encontrar uma partição $V_{1} \cup \ldots \cup V_{t}$ de $V(H)$ com as propriedades garantidas pelo lema. Seja então $H^{*}$ o grafo reduzido de $H$ obtido a partir desta partição. O grafo $H^{*}$ tem pelo menos $(1-\varepsilon)\left(\begin{array}{l}t \\ 2\end{array}\right)$ arestas. Daí, pelo teorema 2.13 (Turán), ele possui um subgrafo completo $H^{* *}$ de ordem (sendo generoso) pelo menos $1 / 2 \varepsilon$. Agora, vamos colorir as arestas de $H^{* *}$ usando as cores verde e laranja. Colorimos $\left(V_{i}, V_{j}\right)$ de verde se $d_{H}\left(V_{i}, V_{j}\right) \geq 1 / 2$ e de laranja se $d_{H}\left(V_{i}, V_{j}\right)<1 / 2$. Daí pelo teorema 2.7 , já que $\frac{1}{2} \log (1 / 2 \varepsilon) \geq d+1$, existe um subgrafo completo $H^{* * *}$ de $H^{* *}$ monocromático com $d+1$ vértices. Podemos assumir sem perda da generalidade que $V\left(H^{* * *}\right)=\left\{V_{1}, \ldots, V_{d+1}\right\}$.

Suponha que $H^{* * *}$ tem todas as suas arestas coloridas de verde. Neste caso, temos que

- $\left(V_{i}, V_{j}\right)$ é $\varepsilon$-regular e

- $d_{H}\left(V_{i}, V_{j}\right) \geq 1 / 2$ 
para todos $i, j$ com $1 \leq i<j \leq d+1$. Neste caso, vamos mostrar que o grafo $H$ das arestas vermelhas contém uma cópia de $G$. O caso em que as arestas de $H^{* * *}$ são todas laranjas é análogo: a segunda condição acima valeria com $\tilde{H}$ no lugar de $H$ e poderíamos então encontrar uma cópia de $G$ em $\tilde{H}$, ou seja, no grafo formado pelas arestas azuis.

Como o número cromático de $G, \chi(G)$, é no máximo $\Delta(G)+1 \leq d+1$, existe uma partição $X_{1} \cup \ldots \cup X_{d+1}$ de $V(G)$, onde cada $X_{i}$ é um conjunto independente. Considere uma enumeração dos vértices de $G, x_{1}, \ldots, x_{n}$, tal que para $1 \leq i_{1}<i_{2} \leq n$ se $x_{i_{1}} \in X_{\beta_{1}}$ e $x_{i_{2}} \in X_{\beta_{2}}$ então $\beta_{1} \leq \beta_{2}$. Vamos agora encontrar uma imersão de $G$ em $H$, ou seja, escolheremos indutivamente vértices $y_{1}, \ldots, y_{n}$ de $H$ de modo que

$$
\left\{x_{i}, x_{j}\right\} \in E(G) \Rightarrow\left\{y_{i}, y_{j}\right\} \in E(H) \text {. }
$$

Suponha que para algum $i, 0 \leq i<n$, os vértices $y_{\alpha}$ tais que $1 \leq \alpha \leq i$ já foram escolhidos satisfazendo as condições a seguir.

1. se $1 \leq \alpha \leq i$ e $x_{\alpha} \in X_{\beta}$, então $y_{\alpha} \in V_{\beta}$.

2. se $i<\alpha^{\prime} \leq n$ e $x_{\alpha^{\prime}} \in X_{\beta}$, sendo $N\left(\alpha^{\prime}, i\right):=\left\{y_{\alpha}: 1 \leq \alpha \leq i,\left\{x_{\alpha}, x_{\alpha^{\prime}}\right\} \in E(G)\right\}$ e $v:=\left|N\left(\alpha^{\prime}, i\right)\right|$, então existe $V_{\beta}^{\prime} \subseteq V_{\beta}$ tal que $\left|V_{\beta}^{\prime}\right| \geq\left|V_{\beta}\right| / 3^{v}$ e todo vértice de $V_{\beta}^{\prime}$ é adjacente $(\mathrm{em} H)$ a todos $y_{\alpha} \in N\left(\alpha^{\prime}, i\right)$.

Mostremos como escolher $y_{i+1}$. Note que, em particular, a construção abaixo vale para $i=0$ e podemos utilizá-la para escolher $y_{1}$. A hipótese (2) acima, apesar de parecer um pouco complicada, é o que se faz necessário para conseguirmos escolher $y_{i+1}$ facilmente. De fato, se $x_{i+1} \in X_{\beta_{0}}$, bastaria escolher $y_{i+1} \in V_{\beta_{0}}^{\prime}$, onde $V_{\beta_{0}}^{\prime}$ é subconjunto de $V_{\beta_{0}}$ dos vértices adjacentes a todos os vértices de $N(i+1, i)$, para que a implicação (3.2) seja válida para os $y_{i}^{\prime} s$ definidos até então e a hipótese (1) acima continuem valendo. Basta então tomar cuidado para garantir que $V_{\beta_{0}}^{\prime}$ não seja vazio e que possamos escolher $y_{i+1} \in V_{\beta_{0}}^{\prime}$ de modo que a hipótese (2) também continue valendo.

Basta olhar apenas para os valores $\alpha^{\prime}$ tais que $\alpha^{\prime}>i+1$ e para os quais $x_{\alpha^{\prime}}$ é adjacente a $x_{i+1}$. É claro que existem no máximo $d$ destes valores. Escolha um deles arbitrariamente, digamos $\alpha^{\prime}$. Seja $\beta$ tal que $x_{\alpha^{\prime}} \in X_{\beta}$. É claro que $\beta>\beta_{0}$. Veja também que $N\left(\alpha^{\prime}, i+1\right)=N\left(\alpha^{\prime}, i\right) \cup x_{i+1}$. Seja então $v^{\prime}=\left|N\left(\alpha^{\prime}, i+1\right)\right|=\left|N\left(\alpha^{\prime}, i\right)\right|+1$. Pela hipótese de indução (2), já sabemos que $V_{\beta}$ contém um subconjunto $V_{\beta}^{\prime}$, de ordem pelo menos $\left|V_{\beta}\right| / 3^{v^{\prime}-1} \geq\left|V_{\beta}\right| / 3^{d}>\varepsilon\left|V_{\beta}\right|$, tal que todo vértice de $V_{\beta}^{\prime}$ é adjacente a todo vértice de $N\left(\alpha^{\prime}, i\right)$. Aplicando a proposição 3.2 , concluímos que no máximo $\varepsilon\left|V_{\beta}\right| \operatorname{dos}$ pontos de $V_{\beta_{0}}^{\prime}$ são adjacentes a menos de um terço dos pontos em $V_{\beta}^{\prime}$ (e não seriam uma escolha viável para $y_{i+1}$ ). Agora, se olharmos para todos os possíveis valores de $\alpha^{\prime}$, no máximo $d \varepsilon\left|V_{\beta_{0}}\right|$ pontos de $V_{\beta_{0}}^{\prime}$ não seriam uma escolha viável para $y_{i+1}$. Contudo também temos de escolher $y_{i+1}$ diferente dos $y_{i}^{\prime} s$ previamente selecionados em $V_{\beta_{0}}^{\prime}$. 
Com isto, eliminamos mais, no máximo, $n$ pontos de $V_{\beta_{0}}^{\prime}$. Assim, para garantir que podemos escolher $y_{i+1}$, basta ver que $\left|V_{\beta_{0}}^{\prime}\right|>d \varepsilon\left|V_{\beta_{0}}\right|+n$. Como $t \leq M$ e $c \geq M / d \varepsilon$, temos $\left|V_{\beta_{0}}\right| \geq c n / t \geq c n / M$, donde $n \leq d \varepsilon\left|V_{\beta_{0}}\right|$. Dessa forma, basta mostrar que $\left|V_{\beta_{0}}^{\prime}\right|>2 d \varepsilon\left|V_{\beta_{0}}\right|$. Mas isto é verdade, já que

$$
\left|V_{\beta_{0}}^{\prime}\right| \geq\left|V_{\beta_{0}}\right| / 3^{v} \geq\left|V_{\beta_{0}}\right| / 3^{d}>2 d \varepsilon\left|V_{\beta_{0}}\right|,
$$

onde $v=|N(i+1, i)|$.

O teorema acima pode ser facilmente adaptado para o caso assimétrico, isto é, dados $G_{1}$ e $G_{2}$ ambos com grau máximo menor ou igual a certo $d$, então

$$
R\left(G_{1}, G_{2}\right) \leq c \max \left\{\left|V\left(G_{1}\right)\right|,\left|V\left(G_{2}\right)\right|\right\},
$$

onde $c$ depende apenas de $d$. De fato, na prova do teorema, a reconstrução do grafo $G$ a partir do subgrafo completo monocromático do grafo reduzido, depende apenas do fato de que o grau máximo de $G$ é menor ou igual a $d$.

Ainda em [5], comenta-se uma outra possível modificação do teorema acima: permitir mais de duas cores. Especificamente, para cada par $(d, r)$ existe uma constante $c$, em função apenas de $d$ e $r$, tal que se $G$ é um grafo com grau máximo $d$ e $n$ vértices então toda $r$-coloração das arestas de um grafo completo com $c n$ vértices possui uma cópia monocromática de $G$. Para tanto é necessário usar a versão do lema da regularidade para mais de uma cor.

Finalmente, podemos considerar as duas generalizações acima ao mesmo tempo, obtendo a seguinte versão do teorema.

Teorema 3.8. Para inteiros positivos $d$ e $r$, existe $c$, função apenas de $d$ e $r$, tal que se $G_{1}, \ldots, G_{r}$ são grafos com grau máximo d então $R(G) \leq c \max \left\{\left|V\left(G_{1}\right)\right|, \ldots,\left|V\left(G_{r}\right)\right|\right\}$. 


\section{Capítulo 4}

\section{Um lema de estabilidade}

Começaremos este capítulo de modo bastante informal. Considere o problema de, dados três grafos $G_{1}, G_{2}$ e $G_{3}$, determinar o número de Ramsey $R\left(G_{1}, G_{2}, G_{3}\right)$. Suponha que conjecturamos que $R\left(G_{1}, G_{2}, G_{3}\right)=N$, para certo natural $N$. Há duas coisas que precisamos mostrar. Primeiro, que existe uma 3-coloração de $K_{N-1}$ tal que $G_{i}$ não é subgrafo do grafo induzido pelas arestas da cor $i$ para todo $1 \leq i \leq 3$. Segundo, que toda 3 -coloração de $K_{N}$ contém uma cópia monocromática de $G_{i}$ na cor $i$ para algum $i$. No primeiro passo, ao buscarmos colorações adequadas de $K_{N-1}$, é muito comum que todas as colorações que conseguimos encontrar sejam parecidas umas com as outras ou que apresentem um mesmo tipo de estrutura. Suponha que conseguimos provar que nenhuma das colorações encontradas para $K_{N-1}$ pode ser estendida a uma 3-coloração de $K_{N}$ que também não contenha uma cópia monocromática de $G_{i}$ na cor $i$ para todo $1 \leq i \leq 3$. Isto é de se esperar, já que tal extensão iria contra a nossa conjectura. Mas é óbvio que isto está longe de ser uma prova de que $R\left(G_{1}, G_{2}, G_{3}\right)=N$, já que poderia existir uma coloração de $K_{N}$ totalmente diferente das que encontramos para $K_{N-1}$ e que não possui uma cópia monocromática de $G_{i}$.

Um lema de estabilidade nos ajudaria a finalizar a prova de nossa conjectura. Ou seja, ele nos diz que, caso exista uma coloração de $K_{N}$ que não possui cópias monocromáticas de $G_{i}$ nas cores adequadas, então tal coloração deve ter o mesmo tipo de estrutura das colorações encontradas para $K_{N-1}$. Para finalizar a prova, bastaria então usar o lema de estabilidade e provar que mesmo as colorações de $K_{N}$ com este tipo de estrutura possuem uma cópia monocromática de $G_{i}$ para algum $i$.

A idéia de usar teoremas de estabilidade em teoria dos grafos não se aplica apenas a problemas em teoria de Ramsey. Ela foi iniciada por Simonovits, ainda nos anos 60 e 70, por exemplo, veja Simonovits [26], Erdôs e Simonovits [8], Lovász e Simonovits [21, 22].

Desde então, esse método tem se mostrado bem sucedido em muitos problemas de teoria extremal, por exemplo, no de encontrar subgrafos maximais livres de triângulos 
em grafos aleatórios [1] e em alguns trabalhos recentes em teoremas extremais exatos para hipergrafos de Füredi e Simonovits [14], Keevash e Sudakov [18], e Füredi, Pikhurko e Simonovits $[12,13]$.

Este capítulo será inteiramente dedicado à exposição da prova de um lema de estabilidade (para emparelhamentos em 3-colorações) de Gyárfás, Ruszinkó, Sárközy e Szemerédi [17]. Este lema será utilizado na prova do teorema 5.1, que é o resultado principal do capítulo 5 e desta dissertação. Apesar do lema de estabilidade ser interessante por si só, sua prova é extensa e técnica e não é necessário conhecê-la para entender a prova do teorema 5.1.

Começamos com algumas definições centrais.

Definição 17. Uma 3-multicoloração de um grafo $G$ é uma família de três conjuntos de arestas de $G,\left\{E_{1}, E_{2}, E_{3}\right\}$, tal que $E(G) \subseteq E_{1} \cup E_{2} \cup E_{3}$. Dizemos que as arestas de $E_{i}$ receberam a cor $i$.

A diferença entre uma 3-multicoloração de um grafo $G$ e uma 3-coloração de $G$ é que na segunda exigimos também que $E_{i} \cap E_{j}=\emptyset, 1 \leq i<j \leq 3$. Já em uma multicoloração algumas arestas podem ter recebido mais de uma cor. Em todo caso, todas as arestas devem receber pelo menos uma das cores. Em geral, chamaremos as cores de 1, 2 e 3 (para enumerá-las com facilidade), mas em alguns casos é mais agradável chamá-las de vermelho, verde e azul.

Definição 18. Dizemos que uma aresta é c-exclusiva ou exclusiva da cor $c$ se ela recebeu apenas a cor c. Denotamos respectivamente por $G^{R}, G^{G}$ e $G^{B}$ os subgrafos induzidos pelas arestas que receberam cor vermelha, verde e azul e por $G^{R^{*}}, G^{G^{*}}$ e $G^{B^{*}}$ os subgrafos induzidos pelas arestas exclusivas dessas cores.

Definição 19. Em uma 3-multicoloração, um subconjunto $A$ de arestas é monocromático se existe uma cor $i$ tal que todas as arestas de $A$ receberam a cor $i$. Veja que as arestas de $A$ não precisam ser exclusivas da cor $i$.

Definição 20. Um emparelhamento conectado em um grafo $G$ é um emparelhamento $M$ cujas arestas estão todas em uma mesma componente conexa de $G$.

Definição 21. Dada uma coloração de um grafo $G$, dizemos que $M$ é um emparelhamento monocromático conectado se existe uma cor $i$ tal que $M \subseteq E\left(G^{i}\right)$ e $M$ é conectado em $G^{i}$.

Também usaremos a seguinte convenção.

Definição 22. Dizemos que um grafo $G$ sobre $n$ vértices é $(1-\varepsilon)$-denso se ele possui pelo menos $(1-\varepsilon)\left(\begin{array}{l}n \\ 2\end{array}\right)$ arestas. Um grafo bipartido com classes de vértices de ordens $k$ e $l$ é $(1-\varepsilon)$-denso se ele possui pelo menos $(1-\varepsilon) k l$ arestas.

O lema de estabilidade nos diz que todas as 3-multicolorações de um grafo quase 
completo, ou seja, com densidade de arestas alta, que não possuem um emparelhamento monocromático conectado grande são do tipo $\mathrm{EC}_{1}, \mathrm{EC}_{2}$ ou $\mathrm{EC}_{3}$, definidas abaixo.

Coloração $1\left(\operatorname{EC}_{1}(\alpha, \delta)\right)$. Uma coloração de $G$ é do tipo $\operatorname{EC}_{1}(\alpha, \delta)$, com $\alpha, \delta<1$, se existe uma partição $V(G)=A \cup B \cup C \cup D$ tal que

- $|A|,|B|,|C|,|D| \geq(1-\alpha) \frac{|V(G)|}{4}$;

- os grafos bipartidos $(A \times B) \cap G^{R^{*}},(C \times D) \cap G^{R^{*}},(A \times D) \cap G^{G^{*}},(B \times C) \cap G^{G^{*}}$, $(A \times C) \cap G^{B^{*}},(B \times D) \cap G^{B^{*}}$ são todos $(1-\delta)$-densos.

Coloração $2\left(\mathrm{EC}_{2}(\alpha, \delta)\right)$. Uma coloração de $G$ é do tipo $\mathrm{EC}_{2}(\alpha, \delta)$, com $\alpha, \delta<1$, se existe uma partição $V(G)=A \cup B \cup C \cup D$ tal que

- $|A|,|B|,|C|,|D| \geq(1-\alpha) \frac{|V(G)|}{4}$;

- os grafos bipartidos $(A \times B) \cap G^{R^{*}},((A \cup B) \times C) \cap G^{G^{*}},((A \cup B) \times D) \cap G^{B^{*}}$ são todos $(1-\delta)$-densos.

Coloração $3\left(\mathrm{EC}_{3}\left(\mu, c_{1}, c_{2}, \delta\right)\right)$. Uma coloração de $G$ é do tipo $\mathrm{EC}_{3}\left(\mu, c_{1}, c_{2}, \delta\right)$, com $\mu, c_{1}, c_{2}, \delta<1$, se existe uma partição $V(G)=A \cup B \cup C \cup D$ tal que

- $|A|,|B|,|C| \geq\left(1-c_{1} \mu\right) \frac{|V(G)|}{4} ; \quad|D| \geq \mu \frac{|V(G)|}{4} ;$

- $|A| \geq \max \{|B|,|C|,|D|\}+\mu \frac{|V(G)|}{4} ; \quad|A \cup D| \leq\left(1+c_{2} \mu\right) \frac{|V(G)|}{2}$

- os grafos bipartidos $(A \times B) \cap G^{R^{*}},(C \times D) \cap G^{R^{*}},(A \times D) \cap G^{G^{*}},(B \times C) \cap G^{G^{*}}$, $(A \times C) \cap G^{B^{*}},(B \times D) \cap G^{B^{*}}$ são todos $(1-\delta)$-densos.

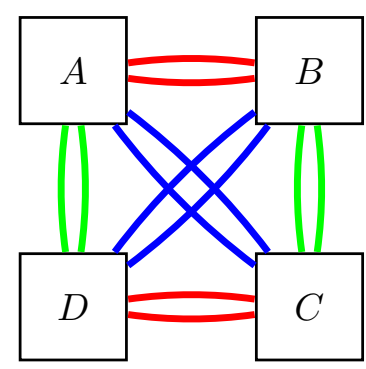

$\mathrm{EC}_{1}$

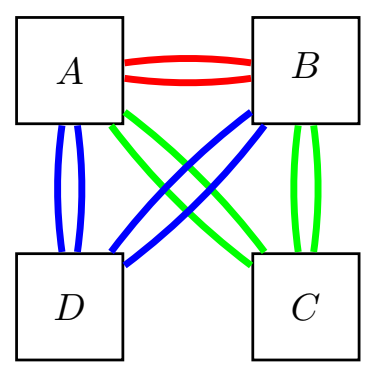

$\mathrm{EC}_{2}$

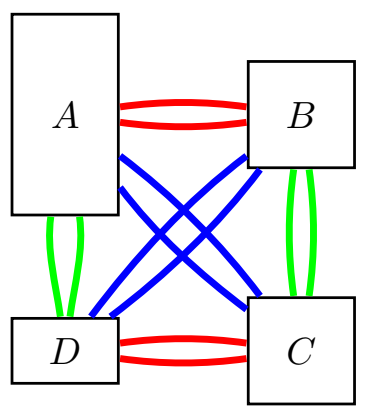

$\mathrm{EC}_{3}$

Observação. Quando omitirmos o parâmetro $\delta$ das colorações $\mathrm{EC}_{1}$ e $\mathrm{EC}_{2}$ é porque estamos supondo que $\delta=\alpha$. Note também que em cada definição exigimos que certas arestas sejam exclusivas de certas cor e não apenas que os conjuntos sejam monocromáticos. 
Agora, podemos enunciar o lema.

Lema 4.1 (Lema 1 em [17], versão corrigida). Dados quaisquer $\alpha_{1}>0$ e $\mu_{1}>0$, existem reais positivos $\eta_{4.1}, \varepsilon_{4.1}$ e $\mu_{4.1}<\mu_{1}$ tais que para todo $\varepsilon<\varepsilon_{4.1}$ existe um inteiro positivo $n_{4.1}$ para o qual vale que: se um grafo $(1-\varepsilon)$-denso $G_{n}$, com $n \geq n_{4.1}$, é 3-multicolorido, então um dos seguintes casos acontece.

Caso 1: $G_{n}$ contém um emparelhamento monocromático conectado com pelo menos $\left(\frac{1}{4}+\eta_{4.1}\right) n$ arestas.

Caso 2: a coloração é do tipo $\operatorname{EC}_{1}\left(\alpha_{1} / 2, \alpha_{1} / 2\right)$.

Caso 3: a coloração é do tipo $\mathrm{EC}_{2}\left(\alpha_{1} / 2, \alpha_{1} / 2\right)$.

Caso 4: a coloração é do tipo $\mathrm{EC}_{3}\left(\mu_{4.1}, 0,7,0,2, \varepsilon^{1 / 3}\right)$.

Observação. Na versão original do lema, o "caso 4" acima não existe. Contudo, este caso é necessário já que, se $\alpha$ é pequeno, então existem colorações que são do tipo $\mathrm{EC}_{3}$ e não se enquadram nos casos 1, 2 e 3. Por exemplo, tome uma coloração do tipo $\mathrm{EC}_{3}$ onde $|B|=|C|=|V(G)| / 4,|A|=3|V(G)| / 8$ e $|D|=|V(G)| / 8$, tal que todas as arestas dentro de $A$ são verdes $e \delta=0$.

A prova original do lema em [17] comete um pequeno descuido no final de seu subcaso 1.2. Ao tentar corrigir esse erro, percebemos que o máximo que podíamos concluir neste subcaso era que a coloração deveria ser do tipo $\mathrm{EC}_{3}$. Por outro lado, a existência do caso 4 acima não afeta a veracidade do resultado principal de [17], já que, ao aplicar o lema, este caso pode ser tratado de maneira semelhante ao caso 2, em que temos uma coloração do tipo $\mathrm{EC}_{1}$.

\subsection{Ferramentas, outros resultados do tipo Ramsey e suas versões aproximadas}

Como o título sugere, nesta seção enunciaremos e provaremos alguns resultados básicos, diversos resultados do tipo Ramsey e versões aproximadas destes. Usaremos todos estes resultados na prova do lema 4.1, exceto um ou dois que foram colocados por caráter ilustrativo.

Definição 23. Denotamos por $\nu=\nu(G)$ o tamanho (número de arestas) de um emparelhamento máximo em um grafo $G$. Denotamos por $c(G)$ o número de componentes de $G$ e por $c_{o}(G)$ o número de componentes ímpares de $G$.

O resultado a seguir é bem conhecido pelo nome de Fórmula de Tutte-Berge. 
Lema 4.2. Para todo grafo $G$,

$$
|V(G)|-2 \nu(G)=\max \left\{c_{o}(G \backslash S)-|S|\right\},
$$

onde o máximo é tomado sobre todos os conjuntos $S \subseteq V(G)$.

Também precisamos da seguinte observação trivial sobre emparelhamentos.

Lema 4.3. Suponha que $M=\left\{e_{1}, \ldots, e_{k}\right\}$ é um emparelhamento máximo em um grafo $G$. Então $V(G) \backslash V(M)$ é um conjunto independente de $G$ e podemos selecionar para cada $i, 1 \leq i \leq k$, uma extremidade $x_{i}$ da aresta $e_{i}$ de modo que exista no máximo uma aresta de $x_{i}$ para $V(G) \backslash V(M)$ em $G$. Dizemos que $x_{i}$ é uma extremidade forte de $e_{i}$.

Os próximos lemas são sobre propriedades simples de grafos com densidade alta.

Lema 4.4. Seja $G_{n}$ um grafo $(1-\varepsilon)$-denso (com $n$ vértices). Então $G_{n}$ possui um subgrafo $H$ com pelo menos $(1-\sqrt{\varepsilon}) n$ vértices tal que: $(A) \Delta(\bar{H})<\sqrt{\varepsilon} n ;(B) \delta(H) \geq$ $(1-2 \sqrt{\varepsilon}) n ;(C) H$ é $(1-2 \sqrt{\varepsilon})$-denso.

Prova. Se $\overline{G_{n}}$ possui $p$ vértices com grau pelo menos $\sqrt{\varepsilon} n$, então $\overline{G_{n}}$ tem pelo menos $p \sqrt{\varepsilon} n / 2$ arestas. Então

$$
\frac{p \sqrt{\varepsilon} n}{2} \leq \varepsilon\left(\begin{array}{l}
n \\
2
\end{array}\right)
$$

o que implica $p<\sqrt{\varepsilon} n$. Afirmamos que o grafo $H$ obtido após a remoção destes $p$ vértices de $G$ é como o desejado. De fato, as propriedades (A) e (B) valem trivialmente e a propriedade $(\mathrm{C})$ segue facilmente de $(\mathrm{B})$, pois

$$
\begin{aligned}
|E(H)| \geq \frac{|V(H)| \delta(H)}{2} & \geq \frac{|V(H)|(1-2 \sqrt{\varepsilon}) n}{2} \geq(1-2 \sqrt{\varepsilon}) \frac{|V(H)|^{2}}{2} \\
& >(1-2 \sqrt{\varepsilon})\left(\begin{array}{c}
|V(H)| \\
2
\end{array}\right) .
\end{aligned}
$$

Notação 24. Dado um grafo $G$ e subconjuntos disjuntos $A$ e $B$ de $V(G)$, o grafo bipartido formado pelo conjunto de vértices $A \cup B$ e cujas arestas possuem uma extremidade em $A$ e a outra em $B$ será denotado por $G[A, B]$ ou simplesmente por $[A, B]$, quando não houver perigo de confusão.

Lema 4.5. Seja $H=[A, B]$ um grafo bipartido tal que $|B| \geq|A|$ e que todo vértice de $A$ é não adjacente a no máximo $k$ vértices (em $B$ ) e que todo vértice de $B$ é não adjacente a no máximo $k$ vértices (em $A$ ). Se $k<|B| / 2$ então $H$ possui uma única componente conexa não trivial, ou seja, todas as arestas de $H$ estão em uma mesma componente conexa. Se também temos que $|A|>k$ então $H$ é conexo e contém um emparelhamento de tamanho pelo menos $|A|-k$. 
Prova. Suponha que $k<|B| / 2$. Neste caso, todo vértice de $A$ é adjacente a mais da metade dos vértices de $B$. Logo, quaisquer dois vértices de $A$ possuem um vizinho comum em $B$, donde todos os vértices de $A$ pertencem a uma mesma componente conexa. É claro que qualquer vértice de $B$ que possui grau não nulo também pertence a esta componente. Logo, existe uma única componente não trivial.

Suponha agora que $|A|>k$. Então $B$ não tem vértices de grau nulo e portanto $H$ é conexo. Finalmente, qualquer emparelhamento máximo $M$ em $H$ cobre todos exceto no máximo $k$ vértices de $A$. De fato, se $M$ deixa de cobrir mais do que $k$ vértices de $A$, então também deixa de cobrir mais do que $k$ vértices em $B$. Tome $v \in A \backslash V(M)$. Pela maximalidade de $M, v$ não é adjacente a todos os vértices de $B \backslash V(M)$, ou seja, não é adjacente a mais do que $k$ vértices de $B$. Uma contradição.

Observação. O lema acima será frequentemente aplicado a subgrafos bipartidos de um grafo $G$ onde $\Delta(\bar{G})<\sqrt{\varepsilon} n$. Assim tomando $k=\sqrt{\varepsilon} n$, temos que se $A$ e $B$ são conjuntos disjuntos de vértices de $G$ tais que $|B|>2 \sqrt{\varepsilon} n$ e $A \neq \emptyset$ então $H=[A, B]$ possui uma única componente conexa. Se adicionalmente $|A|>\sqrt{\varepsilon} n$, então $H$ é conexo e contém um emparelhamento de tamanho pelo menos $|A|-\sqrt{\varepsilon}$.

Como vimos no capítulo 2, o número de Ramsey para emparelhamentos é completamente descrito pelo teorema 2.11. Contudo, este teorema ainda não é forte o bastante para podermos aplicá-lo em uma prova do lema 4.1, pois este lema trata de 3-colorações de grafos densos e não de grafos completos. Pelo que vimos no lema 4.4, um grafo denso $G$ possui um subgrafo grande $H$ tal que $\Delta(\bar{H})$ é pequeno. Pensando em uma 3-coloração de $G$ como uma 4-coloração de um grafo completo (onde a quarta cor equivale às arestas ausentes em $G$ ), o teorema a seguir será bastante útil.

Teorema 4.6 (Theorem 3 in [17]). Para $n_{1}, n_{2}, n_{3} \geq 1$ inteiros com $n_{1}=\max \left\{n_{1}, n_{2}, n_{3}\right\}$ e para todo inteiro não-negativo $s$,

$$
R\left(n_{1} K_{2}, n_{2} K_{2}, n_{3} K_{2}, K_{1, s}\right) \leq s+n_{1}+1+\sum_{i=1}^{3}\left(n_{i}-1\right) .
$$

Prova. Fixe $s \geq 1$. A prova é por indução sobre $n_{1}=\max \left\{n_{1}, n_{2}, n_{3}\right\}$. O resultado é óbvio para $n_{1}=1$. Neste caso, $n_{1}=n_{2}=n_{3}=1$, ou seja, basta observar que $R\left(K_{2}, K_{2}, K_{2}, K_{1, s}\right) \leq s+2$. Suponha então que $n_{1}>1$ e que o resultado foi provado para $n_{1}-1$. Deixamos a prova de que o resultado equivalente para menos de 4 cores vale, ou seja, o caso em que $n_{i}=1$ para algum $i$, a cargo do leitor. Suponha então que $n_{i}>1$ para todo $i$. Seja $N=s+n_{1}+1+\sum_{i=1}^{3}\left(n_{i}-1\right)$ e considere uma 4-coloração de $K_{N}$. Pensaremos na quarta cor como o complemento do grafo $G$ formado pelos $N$ vértices e pelas arestas das cores 1, 2 e 3 . Suponha que existe em $G$ uma árvore $T$, não necessariamente induzida, formada por exatamente 3 arestas (sendo portanto um caminho ou uma estrela) de cores distintas. Como $|G \backslash V(T)|=N-4=$ 
$s+\left(n_{1}-1\right)+1+\sum_{i=1}^{3}\left(\left(n_{i}-1\right)-1\right)$, por hipótese de indução, podemos achar em $G \backslash V(T)$ um emparelhamento de tamanho $n_{i}-1$ na cor $i$ ou podemos achar no complemento de $G \backslash V(T)$ um $K_{1, s}$. No primeiro caso, podemos adicionar a aresta de cor $i$ de $T$ ao emparelhamento encontrado, no segundo, não há o que fazer.

Podemos supor então que $G$ não possui tal árvore, não possui um emparelhamento monocromático de tamanho $n_{i}$ na cor $i$, para $i=1,2$ e 3 , e que cada vértice de $G$ tem grau no mínimo $N-s$. Tome um emparelhamento máximo no grafo $G^{1}$ das arestas de cor 1 , digamos $M_{1}=\left\{e_{1}, \ldots, e_{k_{1}}\right\}$. Usando o lema 4.3, aplicado ao subgrafo induzido pelas arestas da cor 1 , selecione vértices fortes $X=\left\{x_{1}, \ldots, x_{k_{1}}\right\}$, um de cada aresta de $M_{1}$. Seja $H$ o subgrafo de $G$ induzido por $V(G) \backslash V\left(M_{1}\right)$.

Sejam ainda $M_{2}$ e $M_{3}$ emparelhamentos tais que $M_{i}$ é máximo no subgrafo de $G$ induzido por $V(H) \cup X$ e pelas arestas de cor $i$ e que $V\left(M_{i}\right) \cap X$ seja máximo. Sejam $k_{2}=\left|M_{2}\right|$ e $k_{3}=\left|M_{3}\right|$.

Suponha que existe um vértice $x_{1} \in X$ que não pertence a $V\left(M_{2}\right) \cup V\left(M_{3}\right)$. Isto implica que $x_{1}$ é adjacente a no máximo um vértice de $H$ na cor 1 (de acordo com a escolha de $x_{1}$ pelo lema 4.3) e a no máximo $k_{i}$ vértices de $H$ na cor $i$ (devido à maximalidade de $\left|M_{i}\right|$ e de $\left.\left|V\left(M_{i}\right) \cap X\right|\right)$, para $i=2$ e $i=3$. Além disso, há no máximo $s-1$ vértices de $H$ não adjacentes a $x_{1}$. Assim,

$$
N \leq 2 k_{1}+1+k_{2}+k_{3}+(s-1) \leq 2\left(n_{1}-1\right)+\left(n_{2}-1\right)+\left(n_{3}-1\right)+s<N,
$$

o que é uma contradição.

Logo, todo vértice de $X$ é coberto por uma aresta de $M_{2}$ ou $M_{3}$. Assuma, sem perda da generalidade, que $k_{2} \geq k_{3}$. Suponha que um vértice, digamos $x_{1}$, é coberto por uma aresta $\left\{x_{1}, y_{1}\right\}$ de $M_{3}$. Pelo lema 4.3 , aplicado ao subgrafo induzido pelas arestas de cor 3 em $V(H) \cup X$, selecione um vértice forte de cada aresta de $M_{3}$, em particular um vértice $z$ de $\left\{x_{1}, y_{1}\right\}$. Observe que em $G$ não há arestas da cor 2 incidentes a $z$, caso contrário teríamos uma árvore com 3 arestas de cores distintas. Assim, pela escolha de $z$, o vértice $z$ é adjacente em $H$ a no máximo um vértice na cor 1 , nenhum na cor 2 e no máximo $2 k_{3}+1$ vértices na cor 3 . Ademais, $z$ não é adjacente a no máximo $s-1$ vértices de $H$. Então

$$
\begin{aligned}
N & \leq 2 k_{1}+1+\left(2 k_{3}+1\right)+(s-1) \leq 2 k_{1}+k_{2}+k_{3}+s+1 \\
& \leq 2\left(n_{1}-1\right)+\left(n_{2}-1\right)+\left(n_{3}-1\right)+s+1<N,
\end{aligned}
$$

o que é uma contradição.

Concluímos então que nenhum vértice de $X$ é coberto por uma aresta de $M_{3}$ e portanto todos são cobertos por $M_{2}$. Agora, aplicamos o mesmo argumento de antes. Usamos o lema 4.3 para o subgrafo induzido pelas arestas de cor 2 em $V(H) \cup X$, e selecionamos, em particular, um vértice $z$ de uma aresta $\left\{x_{1}, y_{1}\right\}$ de $M_{2}$. Como antes, nenhuma aresta de $G$ da cor 3 é incidente a $z$. E, de acordo com a escolha de $z$, 
temos que $z$ é adjacente em $H$ a no máximo um vértice na cor 1 , no máximo $2 k_{2}-k_{1}+1$ vértices na cor 2 (pois todos os vértices de $X$ são cobertos por $M_{2}$ e logo $|X|=k_{1}$ vértices de $M_{2}$ estão fora de $H$ ), nenhum vértice na cor 3 e no máximo $s-1$ vértices de $H$ não são adjacentes a $z$. Assim,

$$
\begin{aligned}
N & \leq 2 k_{1}+1+2 k_{2}-k_{1}+1+s-1=k_{1}+2 k_{2}+s+1 \\
& \leq\left(n_{1}-1\right)+2\left(n_{2}-1\right)+s+1= \\
& =n_{1}+2 n_{2}-2+s<N,
\end{aligned}
$$

já que $n_{3}>0$, o que é uma contradição mais uma vez.

Observação. O resultado análogo a esse para mais cores também vale. Precisamente, se $n_{1}=\max \left\{n_{1}, \ldots, n_{t}\right\}$, então $R\left(n_{1} K_{2}, \ldots, n_{t} K_{2}, K_{1, s}\right) \leq s+n_{1}+1+\sum_{i=1}^{t}\left(n_{i}-1\right)$. Mas apenas o caso em que $t=3$ será necessário para nós.

Outra observação trivial é que o tamanho de um emparelhamento monocromático conectado máximo em uma coloração pode ser menor do que o tamanho de um emparelhamento monocromático máximo na mesma. Por exemplo, se o $K_{4}$ é 3-colorido de modo que cada classe de cor é formada por duas arestas independentes, então o maior emparelhamento monocromático tem tamanho 2, enquanto que o maior emparelhamento monocromático conectado tem tamanho 1. Agora, vamos olhar para resultados que garantem componentes conexas monocromáticas grandes em certas colorações, para depois usar esses resultados em conjunto com os anteriores.

O número de Ramsey para componentes conexas é bem conhecido. Alguns resultados mais gerais sobre isto podem ser encontrados em [11]. Apenas para ilustrar, citamos um resultado que, de certa forma, motivou a conjectura de Faudree e Schelp que veio a se tornar o teorema 1.4.

Teorema 4.7. $O$ menor $m$ para o qual toda 3 -coloração do $K_{m}$ contém uma componente conexa monocromática com pelo menos $n$ vértices é $2 n-1$ para $n$ ímpar e $2 n-2$ para $n$ par.

Uma observação de Erdôs bastante conhecida é que em toda 2-coloração do $K_{n}$ existe uma componente conexa monocromática que contém todos os vértices do $K_{n}$. Em outras palavras, para qualquer grafo $G$, vale que $G$ ou $\bar{G}$ é conexo. O resultado a seguir é uma versão aproximada dessa observação.

Lema 4.8. Dado um grafo $G_{n}$ de ordem $n$ tal que $\Delta\left(\overline{G_{N}}\right)<k$, para algum $k \in \mathbb{N}$, e uma 2-coloração de suas arestas, então $G_{n}$ contém uma componente conexa monocromática de ordem pelo menos $n-2 k$.

Prova. Suponha que $\left|V\left(G_{n}\right) \backslash C\right|>2 k$ para uma componente conexa monocromática máxima $C$, digamos de cor 1. Então, todas as arestas do grafo bipartido induzido pelas arestas com uma ponta em $C$ e a outra em $V\left(G_{n}\right) \backslash C$ possui cor 2. Logo, o 
lema 4.5 implica que este grafo bipartido possui uma componente que cobre todos os vértices de $C$ e todos exceto no máximo $k$ vértices de $V\left(G_{n}\right) \backslash C$, mas isto contraria a maximalidade de $C$.

Finalmente, o último resultado desta seção é um resultado de estabilidade relacionado a componentes em 2-colorações de um grafo tripartido.

Lema 4.9. Sejam $\sqrt{\varepsilon}<1 / 24$ e $G_{n}$ um grafo (de ordem $n$ ) tal que $\Delta\left(\overline{G_{n}}\right)<\sqrt{\varepsilon}$, e considere uma partição $V_{1} \cup V_{2} \cup V_{3}$ de $V\left(G_{n}\right)$ tal que $\left|V_{i}\right|>4 \sqrt{\varepsilon} n$, para $i=1,2,3$. Seja $H$ o grafo tripatido induzido pelas arestas em $\left[V_{i}, V_{j}\right], i \neq j$. Então, para toda 2-coloração de $H$, ou encontramos uma componente conexa monocromática $C$ que contém todos exceto no máximo $6 \sqrt{\varepsilon} n$ vértices de $H$, ou a coloração é formada apenas por duas componentes $C_{1}, C_{2}$ de cores distintas, tais que $V\left(C_{1}\right)$ contém todos os vértices de duas das classes da partição e $V\left(C_{2}\right)$ também contém todos os vértices dessas duas classes. Veja que, neste caso, a união $V\left(C_{1}\right) \cup V\left(C_{2}\right)$ contém todos os vértices da terceira classe.

Prova. Seja $C_{1}$ a maior componente conexa monocromática de $H$ e suponha sem perda da generalidade que $C_{1}$ é da cor 1 . Note que $\left|C_{1}\right|>n / 3-\sqrt{\varepsilon} n / 2>n / 4$, pois é fácil encontrar uma estrela monocromática com esta quantidade de vértices. De fato, um vértice que está na menor das classes $V_{i}$ tem pelo menos $2 n / 3-\sqrt{\varepsilon} n$ vizinhos nas outras classes, dos quais pelo menos metade são ligados a ele por arestas de mesma cor. Sejam $R_{i}=V_{i} \cap V\left(C_{1}\right)$ e $S_{i}=V_{i} \backslash V\left(C_{1}\right)$. Para $i \neq j$, pela maximalidade de $C_{1}$, não há arestas da cor 1 entre $R_{i}$ e $S_{j}$. Se $V\left(C_{1}\right)$ contém todos os vértices de duas das classes $V_{i}$, digamos $R_{1}=V_{1}$ e $R_{2}=V_{2}$, então, ou $\left|S_{3}\right| \leq 2 \sqrt{\varepsilon} n$ e $C_{1}$ é a componente $C$ procurada, ou $\left|S_{3}\right|>2 \sqrt{\varepsilon} n$ e a segunda condição do lema é satisfeita com $C_{2}=S_{3} \cup R_{1} \cup R_{2}$. De fato, quando $\left|S_{3}\right|>2 \sqrt{\varepsilon} n$, como $\left[S_{3}, R_{1}\right]$ e $\left[S_{3}, R_{2}\right]$ só possui arestas de cor 2 , temos que $\mathrm{C}_{2}$ é conexo pelo lema 4.5. Agora, podemos assumir que pelo menos dois dos conjuntos $S_{i}$ são não vazios, digamos $S_{1}$ e $S_{2}$.

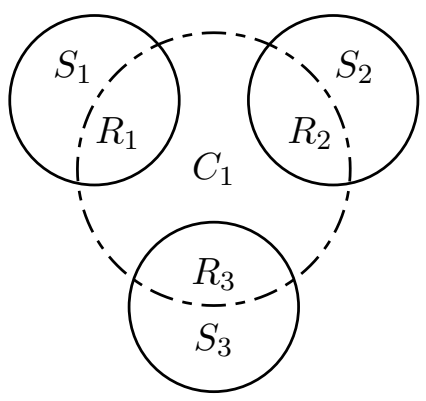

Chame um conjunto de pequeno se ele possui menos que $2 \sqrt{\varepsilon} n$ vértices, e de grande caso contrário. Como $\left|V_{i}\right| \geq 4 \sqrt{\varepsilon} n$, pelo menos um dos conjuntos $R_{i}$ ou $S_{i}$ é grande. Aplicando o lema 4.5 , temos que se $S_{i}$ e $R_{j}(i \neq j)$ são grandes, então o grafo induzido 
pelas arestas da cor 2 entre eles é conexo; se apenas um deles é grande, então existe uma componente conexa na cor 2 que cobre todos seus vértices exceto $\sqrt{\varepsilon} n$ do maior. Com esta observação em mente, vamos analisar casos de acordo com as ordens dos conjuntos $S_{i}$.

Se $S_{1}, S_{2}$ e $S_{3}$ são pequenos, então $C_{1}$ já possui a ordem desejada. De fato

$$
\left|C_{1}\right|=\left|V(H) \backslash S_{1} \cup S_{2} \cup S_{3}\right| \geq(1-6 \sqrt{\varepsilon}) n .
$$

Se exatamente um desses conjuntos, digamos $S_{1}$, é grande, então $R_{2}$ e $R_{3}$ são grandes. O lema 4.5 implica que $C_{2}=S_{1} \cup R_{2} \cup R_{3}$ é conexo na cor 2. Se $R_{1}$ for pequeno, então $C_{2}$ é a componente $C$ procurada, já que a mesma estimativa feita acima para $C_{1}$ vale para $C_{2}$. Por outro lado, se $R_{1}$ é grande, lembre que $S_{2}$ ou $S_{3}$ é não vazio, digamos $S_{2}$. Um vértice de $S_{2}$ é vizinho na cor 2 de todos exceto no máximo $\sqrt{\varepsilon} n$ vértices de $R_{1}$ e todos exceto no máximo $\sqrt{\varepsilon} n$ vértices de $R_{3}$. Assim, todos exceto no máximo $\sqrt{\varepsilon} n$ vértices de $R_{1}$ são conectados a $C_{2}$. Temos então uma componente da cor 2 que cobre todos os vértices de $H$, exceto no máximo $\sqrt{\varepsilon} n$ vértices de $V_{1}$ e dos vértices em $S_{2}$ ou $S_{3}$. Logo, tal componente tem pele menos $(1-3 \sqrt{\varepsilon}) n$ vértices.

Se exatamente dois dos conjuntos $S_{i}$, digamos $S_{1}$ e $S_{2}$, são grandes, então $R_{3}$ é grande. Novamente pelo lema 4.5, $C_{3}=S_{1} \cup S_{2} \cup R_{3}$ é conexo na cor 2. Agora, todo vértice de $R_{2}$ é vizinho na cor 2 de todos exceto no máximo $\sqrt{\varepsilon} n$ vértices de $S_{1}$. Em particular, como $S_{1}$ é grande, todo vértice de $R_{2}$ é vizinho de algum vértice de $S_{1}$ na cor 2. O mesmo vale para o par $R_{1}, S_{2}$. Logo, $R_{1}, R_{2}$ estão na mesma componente que $C_{3}$. Assim, $C=C_{3} \cup R_{1} \cup R_{2}$ é conexo na cor 2 e contém todos os vértices de $H$, exceto os de $S_{3}$, que é pequeno.

Por último, temos o caso em que $S_{1}, S_{2}$ e $S_{3}$ são todos grandes. Usamos então o fato de que o maior dos $R_{i}$, digamos $R_{1}$, é grande, já que

$$
\left|R_{1}\right| \geq \frac{1}{3}\left|C_{1}\right| \geq \frac{n}{12} \geq 2 \sqrt{\varepsilon} n,
$$

pois $\sqrt{\varepsilon}<1 / 24$. Então $R_{1} \cup S_{2} \cup S_{3}$ é conexo na cor 2 . Seja $C$ a componente de cor 2 que contém $R_{1} \cup S_{2} \cup S_{3}$. Como no caso anterior, todo vértice de $R_{2} \cup R_{3}$ é, em particular, adjacente a algum vértice de $S_{2} \cup S_{3}$ e portanto pertence a $C$. Ademais, um vértice $v \in R_{2} \cup R_{3} \neq \emptyset$ é adjacente a todos exceto no máximo $\sqrt{\varepsilon} n$ vértices de $S_{1}$. Neste caso, $C$ contém todos exceto no máximo $\sqrt{\varepsilon} n$ vértices de $H$ e o lema está provado.

\subsection{Prova do lema 4.1}

Prova. Comece escolhendo $\eta=\eta_{4.1}$ suficientemente pequeno em relação a $\alpha_{1}$ e $\mu_{1}$. E, em seguida, escolha $\varepsilon$ suficientemente pequeno em relação a $\eta$. 
Considere uma 3-multicoloração de um grafo $(1-\varepsilon)$-denso $G_{n}$, com $n$ grande. Usando o lema 4.4, encontramos um subgrafo $G=G_{N}$ de $G_{n}$, onde $N \geq(1-\sqrt{\varepsilon}) n$ e $\Delta\left(\overline{G_{N}}\right)<\sqrt{\varepsilon} n$. Note que, para provar que a coloração de $G_{n}$ é uma $\mathrm{EC}_{1}$ ou $\mathrm{EC}_{2}$, basta mostrar que a coloração restrita a $G_{N}$ é respectivamente uma $\mathrm{EC}_{1}$ ou $\mathrm{EC}_{2}$ (com parâmetro $\alpha$ um pouco menor), já que os demais $\sqrt{\varepsilon} n$ vértices podem ser distribuídos facilmente. Do mesmo modo, quando provarmos que a coloração restrita a $G_{N}$ é uma $\mathrm{EC}_{3}$, também conseguiremos mostrar que a coloração original é uma $\mathrm{EC}_{3}$ de $G_{n}$ (isso será feito explicitamente após encontrarmos a uma $\mathrm{EC}_{3}$ em $G_{N}$ ). Durante toda esta seção, $\varepsilon, \eta, n, N$ estarão fixados assim como $G_{n}, G_{N}$ e a 3 -multicoloração.

O objetivo então é mostrar que a coloração de $G_{N}$ é do tipo $\mathrm{EC}_{1}$ ou $\mathrm{EC}_{2}$ ou $\mathrm{EC}_{3}$ no caso em que $G_{N}$ não possui um emparelhamento monocromático conectado de tamanho $(1 / 4+\eta) n$. No decorrer da prova, serão definidos dois tipos de estruturas intermediárias que nos ajudarão a alcançar nosso objetivo. A saber, a 4-partição e a coloração extremal fraca, $\mathrm{WEC}_{2}$. Começamos definindo as 4-partições. Depois, provaremos um lema que nos permitirá dividir a prova em dois casos, que serão tratados nas duas subseções seguintes. Apenas no segundo caso precisaremos definir a $\mathrm{WEC}_{2}$. Em cada caso, precisaremos analisar alguns subcasos.

Definição 25. Dizemos que uma 3-multicoloração de $G_{N}$ tem uma 4-partição se $V\left(G_{N}\right)$ pode ser particionado em conjuntos $S, X_{1}, \ldots, X_{4}$ tais que: $|S| \leq 4 \sqrt{\varepsilon} n$, todas as arestas de $\left[X_{1}, X_{2}\right]$ e $\left[X_{3}, X_{4}\right]$ são exclusivas da cor 1 , todas as arestas de $\left[X_{1}, X_{3}\right]$ e $\left[X_{2}, X_{4}\right]$ são exclusivas da cor 2 e todas as arestas de $\left[X_{1}, X_{4}\right]$ e $\left[X_{2}, X_{3}\right]$ são exclusivas da cor 3. Além disso, exigimos que todos os grafos bipartidos acima sejam conexos. Finalmente, exigimos também que todos os $X_{i}$ sejam não vazios e todos exceto no máximo um deles satisfaçam $\left|X_{i}\right|>2 \sqrt{\varepsilon}$.

$\mathrm{O}$ esquema de cores de uma 4-partição é semelhante ao da $\mathrm{EC}_{1}$ e da $\mathrm{EC}_{3}$, porém relaxamos substancialmente as restrições sobre as ordens dos conjuntos envolvidos. $\mathrm{O}$ lema a seguir mostra que sempre é possível encontrar uma 4-partição ou um emparelhamento monocromático conectado grande se a coloração não possuir uma componente conexa monocromática grande.

Afirmação 4.10. Se todas as componentes conexas monocromáticas de $G_{N}$ têm menos do que $\left(\frac{3}{4}-\eta-6 \sqrt{\varepsilon}\right) n$ vértices, então, ou existe um emparelhamento monocromático conectado de tamanho $\left(\frac{1}{4}+\eta\right) n$, ou a 3-multicoloração de $G_{N}$ possui uma 4-partição.

Prova. Suponha que toda componente conexa monocromática de $G_{N}$ tenha ordem menor que $\left(\frac{3}{4}-\eta-6 \sqrt{\varepsilon}\right) n$. Sejam $C_{1}$ e $C_{2}$ componentes conexas monocromáticas de cores distintas, digamos $C_{i}$ da cor $i(i=1,2)$, tais que $V\left(C_{1}\right) \cap V\left(C_{2}\right)$ é não vazio e $\left|V\left(C_{1}\right) \cup V\left(C_{2}\right)\right|$ é máximo. Sejam $X_{1}=V\left(C_{1}\right) \cap V\left(C_{2}\right), X_{2}=V\left(C_{1}\right) \backslash V\left(C_{2}\right)$, $X_{3}=V\left(C_{2}\right) \backslash V\left(C_{1}\right)$ e $X_{4}=V\left(G_{N}\right) \backslash\left(V\left(C_{1}\right) \cup V\left(C_{2}\right)\right)$ (veja a figura seguinte). Observe que, pela escolha de $C_{1}$ e $C_{2}$, todas as arestas em $\left[X_{1}, X_{4}\right]$ e em $\left[X_{2}, X_{3}\right]$ são exclusivas da cor 3 . 


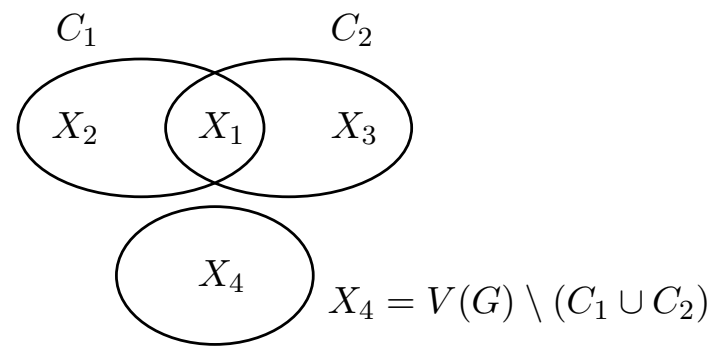

A idéia da prova é que, se $X_{4}$ for pequeno, então $X_{2}$ e $X_{3}$ serão grandes e conseguimos achar o emparelhamento conectado entre eles usando o lema 4.5. E, se $X_{4}$ não for pequeno, conseguimos achar uma 4-partição aplicando algumas vezes o lema 4.5. Sejamos mais precisos agora.

Se $\left|X_{4}\right| \leq 4 \sqrt{\varepsilon} n$, então, como $\left|C_{1}\right|<\left(\frac{3}{4}-\eta-6 \sqrt{\varepsilon}\right) n$, temos que $\left|X_{3}\right|=\left|V\left(G_{N}\right)\right|-$ $\left|C_{1}\right|-\left|X_{4}\right| \geq\left(\frac{1}{4}+\eta+\sqrt{\varepsilon}\right) n$. Analogamente, $\left|X_{2}\right| \geq\left(\frac{1}{4}+\eta+\sqrt{\varepsilon}\right) n$. Logo, o lema 4.5 nos dá um emparelhamento monocromático conectado na cor 3 do tamanho desejado no grafo bipartido $\left[X_{2}, X_{3}\right]$.

Então, podemos supor que $\left|X_{4}\right|>4 \sqrt{\varepsilon} n$. Novamente, aplicando o lema 4.5, dessa vez para o subgrafo induzido pelas arestas entre $X_{1}$ e $X_{4}$, mesmo não tendo uma cota inferior para $\left|X_{1}\right|$, achamos um subgrafo conexo (monocromático de cor 3 ) $\left[X_{1}, X_{4}^{*}\right]$, onde $X_{4}^{*} \subset X_{4}$ e $\left|X_{4}\right|-\left|X_{4}^{*}\right|<\sqrt{\varepsilon} n$. Como $\left|V\left(C_{1}\right)\right|,\left|V\left(C_{2}\right)\right| \geq\left|V\left(\left[X_{1}, X_{4}^{*}\right]\right)\right|$, segue que $\left|X_{2}\right|,\left|X_{3}\right| \geq\left|X_{4}^{*}\right| \geq 3 \sqrt{\varepsilon} n$. Assim, pelo lema 4.5, $\left[X_{2}, X_{3}\right]$ também é conexo na cor 3. Se existir uma aresta da cor 3 em $\left[X_{1} \cup X_{4}^{*}, X_{2} \cup X_{3}\right]$, temos uma componente de cor 3 que contém quase todos os vértices de $G_{N}$. Logo, tal aresta não pode existir e, novamente pela escolha de $C_{1}$ e $C_{2}$, todas as arestas de $\left[X_{1}, X_{3}\right]$ e de $\left[X_{2}, X_{4}^{*}\right]$ são exclusivas da cor 2 e todas as arestas de $\left[X_{1}, X_{2}\right]$ e $\left[X_{3}, X_{4}^{*}\right]$ são exclusivas da cor 1. Aplicando o lema 4.5 para estes subgrafos bipartidos de cor 1 e cor 2 e removendo no máximo $\sqrt{\varepsilon} n$ vértices de $X_{2}$ e de $X_{3}$ (já que não temos cota inferior para $\left|X_{1}\right|$ ), o que encontramos é um subgrafo de $G_{N}$ induzido pela união de conjuntos $X_{1}=X_{1}^{*}, X_{2}^{*}, X_{3}^{*}$ e $X_{4}^{*}$ tais que cada grafo $\left[X_{i}^{*}, X_{j}^{*}\right](i \neq j)$ é conexo e suas arestas são exclusivas da cor desejada. Ademais, $\left|X_{i}^{*}\right|>2 \sqrt{\varepsilon} n$ para $i=2,3,4, X_{1}^{*} \neq \emptyset$ e $S=V\left(G_{N}\right) \backslash \bigcup_{i=1}^{4} X_{i}^{*}$ satisfaz $|S| \leq 4 \sqrt{\varepsilon} n$.

Já que se não existe uma componente conexa monocromática grande então encontramos um emparelhamento conexo monocromático do tamanho desejado em $G_{N}$ (e encerramos a prova) ou encontramos uma 4-partição, podemos tratar dois casos: ou existe uma 4-partição de $G_{N}$, ou $G_{N}$ possui uma componente conexa monocromática grande. Estes casos são tratados nas subseções seguintes. 


\subsubsection{Caso 1 da prova: existe uma 4-partição}

Caso 1: existe uma 4-partição $S \cup X_{1} \cup X_{2} \cup X_{3} \cup X_{4}$ de $V\left(G_{N}\right)$.

Tratamos alguns subcasos em função da ordem dos conjuntos $X_{i}$. O objetivo é encontrar um emparelhamento monocromático conectado ou provar que a coloração é do tipo $\mathrm{EC}_{1}, \mathrm{EC}_{2}$ ou $\mathrm{EC}_{3}$. Para esses subcasos seja

$$
S^{*}=V\left(G_{n}\right) \backslash\left(X_{1} \cup X_{2} \cup X_{3} \cup X_{4}\right)=S \cup\left(V\left(G_{n}\right) \backslash V\left(G_{N}\right)\right) .
$$

É claro que $\left|S^{*}\right| \leq 5 \sqrt{\varepsilon} n$.

Subcaso 1.1: $\left|X_{i}\right| \geq\left(\frac{1}{4}-10 \eta\right) n$ para todo $i=1, \ldots, 4$, ou seja, os conjuntos $X_{i}$ possuem basicamente o mesmo tamanho.

Este caso é fácil. Adicionando os vértices de $S^{*}$ a um dos conjuntos $X_{i}$, digamos $X_{1}$, obtemos uma $\mathrm{EC}_{1}$ com parâmetro $\alpha=40 \eta<\alpha_{1} / 2$ e $A=X_{1} \cup S^{*}, B=X_{2}$, $C=X_{3}, D=X_{4}$. É fácil verificar que as condições de densidade são satisfeitas, já que $\varepsilon<<\alpha$.

Subcaso 1.2: existe $i$ tal que $\left|X_{i}\right|<\left(\frac{1}{4}-10 \eta\right) n$ e, para todo $i,\left|X_{i}\right| \geq 10 \eta n$.

Seja $m_{i}=\left|X_{i}\right|$ para todo $i$. Suponha sem perda da generalidade que $m_{4}$ é o maior e $m_{1}$ é o menor dentre $m_{1}, \ldots, m_{4}$. Como

$$
m_{1}+m_{2}+m_{3}+m_{4} \geq(1-5 \sqrt{\varepsilon}) n \geq(1-\eta / 2) n \quad \text { e } \quad m_{1}<\left(\frac{1}{4}-10 \eta\right) n,
$$

segue que

$$
m_{4} \geq\left(\frac{1}{4}+3 \eta\right) n
$$

Agora, se $m_{3} \geq\left(\frac{1}{4}+2 \eta\right) n$ então podemos usar o lema 4.5 e achar um emparelhamento conexo monocromático entre $X_{3}$ e $X_{4}$ de tamanho pelo menos

$$
\left(m_{3}-\sqrt{\varepsilon} n\right) \geq\left(\frac{1}{4}+2 \eta-\sqrt{\varepsilon}\right) n>\left(\frac{1}{4}+\eta\right) n .
$$

Podemos então supor que

$$
m_{2} \leq m_{3}<\left(\frac{1}{4}+2 \eta\right) n
$$


Aplique agora o teorema $4.6 \operatorname{com} n_{i}=\left(\frac{1}{4}+2 \eta\right) n-m_{i}>0$, para $i=1,2,3$, e $s=\sqrt{\varepsilon} n$. Veja que

$$
\begin{aligned}
R\left(n_{1} K_{2}, n_{2} K_{2}, n_{3} K_{2}, K_{1, s}\right)< & s+n_{1}+\sum_{i=1}^{3} n_{i}= \\
= & \sqrt{\varepsilon} n+2\left(\left(\frac{1}{4}+2 \eta\right) n-m_{1}\right) \\
& +\left(\left(\frac{1}{4}+2 \eta\right) n-m_{2}\right)+\left(\left(\frac{1}{4}+2 \eta\right) n-m_{3}\right) \\
\leq & \sqrt{\varepsilon} n+n-2 m_{1}-m_{2}-m_{3}+8 \eta n \\
\leq & m_{4}+6 \sqrt{\varepsilon} n-m_{1}+8 \eta n \\
< & m_{4} .
\end{aligned}
$$

Logo, como $\Delta\left(\overline{G_{N}}\right)<s$, podemos encontrar dentro de $X_{4}$ um emparelhamento monocromático $N_{i}$ de tamanho $n_{i}$ na cor $i$ para algum $i$. Se $\left|X_{4} \backslash V\left(N_{i}\right)\right| \geq\left|X_{i}\right|$, ou seja, $m_{4}-2 n_{i} \geq m_{i}$, aplicando o lema 4.5 para $\left[X_{4} \backslash V\left(N_{i}\right), X_{i}\right]$, podemos achar um emparelhamento $M_{i}$ de tamanho pelo menos $m_{i}-\sqrt{\varepsilon} n$. Claramente, o emparelhamento $M_{i} \cup N_{i}$ é monocromático. Para mostrar que ele é o emparelhamento que procuramos, basta ver que ele de fato é conectado, pois $\left[X_{4}, X_{i}\right]$ é conexo e todas as arestas de $X_{4}$ para $X_{i}$ possuem a mesma cor, e que

$$
\left|M_{i} \cup N_{i}\right| \geq\left(\left(\frac{1}{4}+2 \eta\right) n-m_{i}\right)+\left(m_{i}-\sqrt{\varepsilon} n\right)>\left(\frac{1}{4}+\eta\right) n .
$$

Logo, resta o caso em que $m_{4}-2 n_{i}<m_{i}$. Isto equivale a

$$
m_{4}+m_{i}<\left(\frac{1}{2}+4 \eta\right) n
$$

Temos então que, se $1 \leq i, j, k \leq 3$ são distintos, então

$$
m_{j}+m_{k} \geq(1-5 \sqrt{\varepsilon}) n-\left(m_{4}+m_{i}\right) \geq\left(\frac{1}{2}-5 \eta\right) n .
$$

Segue que

$$
m_{j}, m_{k} \geq\left(\frac{1}{4}-7 \eta\right) n
$$

Se $j=1$ ou $k=1$ então caímos no caso 1.1. Logo, podemos assumir que $i=1, j=2$ e $k=3$. Se $m_{4} \leq\left(\frac{1}{4}+13 \eta\right) n$, então $m_{1} \geq\left(\frac{1}{4}-18 \eta\right) n$ e novamente podemos achar uma $\mathrm{EC}_{1}$, de modo análogo ao caso 1.1. Senão, então $m_{4}>\left(\frac{1}{4}+13 \eta\right) n$.

Neste caso, temos uma $\mathrm{EC}_{3} \operatorname{com} \mu=\mu_{4.1}:=40 \eta, c_{1}=0,7, c_{2}=0,2$, e $\delta=\varepsilon^{1 / 3} \mathrm{e}$ $A=X_{4}, B=X_{2} \cup S^{*}, C=X_{3}$ e $D=X_{1}$. De fato, as condições sobre as ordens dos conjuntos $A, B, C$ e $D$ são triviais e as condições sobre as densidades também são fáceis 
de verificar. Por exemplo, para obter a cota da densidade das arestas vermelhas em $[A, B]$, veja que todas as arestas em $\left[X_{4}, X_{2}\right]$ são exclusivas da cor 2 , que representará a cor vermelha da $\mathrm{EC}_{3}$, e que há no máximo $\left|X_{4}\right| \sqrt{\varepsilon} n$ pares de vértices não adjacentes neste grafo. Assim, mesmo que não existam arestas da cor $2 \mathrm{em}\left[X_{4}, S^{*}\right]$, a densidade de arestas exclusivas da cor 2 em $[A, B]$ é pelo menos

$$
\frac{\left|X_{4}\right|\left(|B|-\left|S^{*}\right|-\sqrt{\varepsilon} n\right)}{\left|X_{4}\right||B|} \geq \frac{|B|-6 \sqrt{\varepsilon} n}{|B|}=1-\frac{6 \sqrt{\varepsilon} n}{|B|} \geq(1-30 \sqrt{\varepsilon}),
$$

já que $|B| \geq\left|X_{2}\right| \geq n / 5$, pois $\eta$ é pequeno. Finalmente, $(1-30 \sqrt{\varepsilon})>\left(1-\varepsilon^{1 / 3}\right)$, já que $\varepsilon$ é pequeno. As cotas paras as demais densidades seguem de maneira semelhante.

Subcaso 1.3: existe $i$ tal que $\left|X_{i}\right|<10 \eta n$.

Lembre-se que também podemos assumir que toda componente conexa monocromática tem ordem menor que $\left(\frac{3}{4}-\eta-6 \sqrt{\varepsilon}\right) n$. Remova os vértices do conjunto $X_{i}$ de menor cardinalidade, digamos $X_{1}$. Claramente,

$$
\left|X_{2}\right|+\left|X_{3}\right|+\left|X_{4}\right|>n-5 \sqrt{\varepsilon} n-10 \eta n \geq n-11 \eta n .
$$

Assuma que $\left|X_{2}\right| \leq\left|X_{3}\right| \leq\left|X_{4}\right|$.

Note que se $\left|X_{2}\right|<\left(\frac{1}{4}-11 \eta\right) n$, então $V\left(\left[X_{3}, X_{4}\right]\right)>3 n / 4$, mas daí usando o lema 4.5 concluímos que $\left[X_{3}, X_{4}\right]$ possui uma componente monocromática maior que $\left(\frac{3}{4}-\eta-6 \sqrt{\varepsilon}\right) n$, contrariando a hipótese do caso 1 . Além disso, se $\left|X_{3}\right| \geq\left(\frac{1}{4}+2 \eta\right) n$, como no subcaso anterior, conseguimos encontrar um emparelhamento monocromático conectado de tamanho pelo menos $\left(\frac{1}{4}+\eta\right) n$ em $\left[X_{3}, X_{4}\right]$. Assim,

$$
\left(\frac{1}{4}-11 \eta\right) n \leq\left|X_{2}\right| \leq\left|X_{3}\right| \leq\left(\frac{1}{4}+2 \eta\right) n .
$$

Como $\left|X_{4}\right|=\left|V\left(G_{N}\right)\right|-\left|X_{1}\right|-\left|X_{2}\right|-\left|X_{3}\right|$, segue também que

$$
\left(\frac{1}{2}-15 \eta\right) n \leq\left|X_{4}\right| \leq\left(\frac{1}{2}+22 \eta\right) n
$$

Neste caso, conseguiremos ou encontrar um emparelhamento como o desejado, ou mostrar que a coloração é do tipo $\mathrm{EC}_{2}$. Sabemos que, por se tratar de uma 4-partição, todas as arestas de $\left[X_{2}, X_{4}\right]$ são exclusivas da cor 2 , todas as de $\left[X_{3}, X_{4}\right]$ são exclusivas da cor 1 e todas as de $\left[X_{2}, X_{3}\right]$ são exclusivas da cor 3 .

A estratégia agora é semelhante à do final do subcaso anterior. Se $X_{4}$ contém mais que $13 \eta n^{2}$ arestas na cor 1 , então ele contém um emparelhamento $M_{1}$ (não necessariamente conectado) de tamanho $13 \eta n$ na cor 1. Agora, pelo lema 4.5, existe um emparelhamento monocromático conectado $M_{2}$ em $\left[X_{4} \backslash V\left(M_{1}\right), X_{3}\right]$ de tamanho $\left(\left|X_{3}\right|-\sqrt{\varepsilon}\right) n \geq\left(\frac{1}{4}-11 \eta-\sqrt{\varepsilon}\right) n \geq\left(\frac{1}{4}-12 \eta\right) n$. Daí, $M_{1} \cup M_{2}$ é o emparelhamento que estamos procurando. 
Assim, podemos assumir que $X_{4}$ possui no máximo $13 \eta n^{2}$ arestas da cor 1 . Do mesmo modo, podemos assumir que $X_{4}$ possui no máximo $13 \eta n^{2}$ arestas da cor 2 . Mas daí todas exceto no máximo $26 \eta n^{2}$ arestas de $X_{4}$ são exclusivas da cor 3 . O número de vértices fora de $X_{2} \cup X_{3} \cup X_{4}$ é no máximo $\left|X_{1}\right|+5 \sqrt{\varepsilon} n<11 \eta n$. Redistribuindo esses vértices para $X_{2}, X_{3}$ e $X_{4}$, e escolhendo $\alpha=40 \eta<\alpha_{1} / 2$ (com folga) obtemos uma coloração $\mathrm{EC}_{2}$ de $G_{n}$ com parâmetro $\alpha$. Aqui, $X_{4}$ faz o papel de $A \cup B, X_{3}$ faz o papel de $C$ e $X_{2}$ faz o papel de $D$.

\subsubsection{Caso 2 da prova: existe uma componente monocromática grande}

Caso 2: $G_{N}$ possui uma componente monocromática de ordem pelo menos

$$
\left(\frac{3}{4}-\eta-6 \sqrt{\varepsilon}\right) n
$$

Para tratar deste caso, definimos um novo tipo de coloração, a $\mathrm{WEC}_{2}$, obtida através de uma relaxação da coloração $\mathrm{EC}_{2}$. Esta coloração terá um papel semelhante ao das 4-partições do caso anterior. O lema 4.11 abaixo nos diz que achar uma coloração $\mathrm{WEC}_{2}$ é suficiente para terminar a prova do lema de estabilidade.

Coloração 4 ( $\mathrm{WEC}_{2}$ (com parâmetro $\left.\beta\right)$ ). Uma coloração de $G_{N}$ é do tipo $\mathrm{WEC}_{2}$ se existem subconjuntos disjuntos $A, B$ e $C$ de $V\left(G_{N}\right)$, um conjunto $Q \subset A \cup B$ e duas cores $i, j$ tais que

- $|A|,|B|,|C| \geq(1-\beta) \frac{n}{4}$ e $|Q|<(1+\beta) \frac{n}{4}$ (onde $\left.N>(1-\sqrt{\varepsilon}) n\right)$;

- o grafo bipartido $G[A \cup B, C]$ é colorido apenas com as cores $i$ e $j$;

- o grafo bipartido $G[A, B]$ é colorido apenas com a cor $i$, exceto pelas arestas dentro de $Q$, que são coloridas com a cor $i$ ou com a cor $j$.

Lema 4.11. Dados $\beta, \eta, \varepsilon>0$ tais que $\varepsilon$ é suficientemente menor que $\eta$, existe $\alpha$ que é combinação linear de $\beta, \eta$ e $\varepsilon$ com coeficientes constantes, tal que o seguinte é verdadeiro. Se uma 3-multicoloração de um grafo $G_{N}$, onde $\Delta\left(\overline{G_{N}}\right)<\sqrt{\varepsilon}$ é do tipo $\mathrm{WEC}_{2}$ com parâmetro $\beta$, então $G_{N}$ possui um emparelhamento monocromático conectado de tamanho $\left(\frac{1}{4}+\eta\right) n$ ou esta é uma coloração do tipo $\mathrm{EC}_{2}$ com parâmetro $\alpha$.

A prova deste lema será postergada para a próxima subseção.

Começamos a análise do caso 2 definindo componentes conexas maximais $C_{1}, C_{2}$ e alguns emparelhamentos maximais nestas componentes. Sabemos que a maior componente de $G_{N}$ tem ordem pelo menos

$$
\left(\frac{3}{4}-\eta-6 \sqrt{\varepsilon}\right) n>\left(\frac{3}{4}-2 \eta\right) n
$$


Seja $C_{1}$ a maior componente monocromática de $G_{N}$ e seja $\left|C_{1}\right|=\left(\frac{3}{4}-2 \eta+\gamma\right) n$ (com $\left.0 \leq \gamma \leq \frac{1}{4}+2 \eta\right)$. Digamos que $C_{1}$ é da cor verde. Seja $M_{1}$ o maior emparelhamento de $C_{1}$. Note que $M_{1}$ é conectado por definição, então podemos assumir que $\left|M_{1}\right|=k_{1}=\left(\frac{1}{4}+\eta-\rho_{1}\right) n$, onde $0<\rho_{1} \leq \frac{1}{4}+\eta$. Usando o lema 4.3, selecione uma extremidade forte de cada aresta de $M_{1}$ e considere o conjunto $V_{1}=\left\{x_{1}, \ldots, x_{k_{1}}\right\}$ dessas extremidades. Sejam $V_{0}=V\left(M_{1}\right) \backslash V_{1}, V_{2}=V\left(C_{1}\right) \backslash V\left(M_{1}\right)$ e $V_{3}=V\left(G_{N}\right) \backslash V\left(C_{1}\right)$, como na figura abaixo. Seja $H$ o subgrafo definido pela união do subgrafo tripartido quase completo induzido pelas classes $V_{i}$, para $i=1,2,3$, com as arestas internas à classe $V_{2}$. Note que, após removermos no máximo uma aresta da cor verde incidente a cada vértice de $V_{1}$, todas as arestas restantes em $H$ são da cor vermelha ou azul. Para manter a notação simples, continuamos a chamar de $H$ o grafo obtido após a remoção dessas arestas. Temos agora que

$$
\begin{aligned}
& \left|V_{0}\right|=\left|V_{1}\right|=k_{1}=\left(\frac{1}{4}+\eta-\rho_{1}\right) n, \\
& \left|V_{2}\right|=\left(\frac{1}{4}+\gamma-4 \eta+2 \rho_{1}\right) n, \\
& \left(\frac{1}{4}+2 \eta-\gamma-\sqrt{\varepsilon}\right) n \leq\left|V_{3}\right| \leq\left(\frac{1}{4}+2 \eta-\gamma\right) n . \\
& \underbrace{\overbrace{V_{1}}^{V_{0}}}_{C_{1}}
\end{aligned}
$$

Iremos agora definir uma componente monocromática $C_{2}^{\prime}$, mas o modo como $C_{2}^{\prime}$ será definida dependerá da ordem dos conjuntos $V_{i}$. Se $\left|V_{3}\right| \leq 4 \sqrt{\varepsilon} n$, então aplicamos o lema 4.8 ao subgrafo de $H$ induzido por $V_{2}$ e selecionamos uma componente $C_{2}^{\prime}$ monocromática que contém todos exceto no máximo $\sqrt{\varepsilon} n$ vértices de $V_{2}$. Caso contrário, quando $\left|V_{3}\right|>4 \sqrt{\varepsilon} n$, temos ainda duas possibilidade. Se

$$
\left|V_{1}\right|=\frac{1}{4}+\eta-\rho_{1} \geq 4 \sqrt{\varepsilon} \quad \text { e } \quad\left|V_{2}\right|=\left(\frac{1}{4}+\gamma-4 \eta+2 \rho_{1}\right) \geq 4 \sqrt{\varepsilon},
$$

então podemos aplicar o lema 4.9 para definir $C_{2}^{\prime}$, ou como a componente que cobre todos exceto no máximo $6 \sqrt{\varepsilon} n$ vértices de $H$, ou como a maior das componentes que cobre dois dos $V_{i}$. Caso contrário defina $C_{2}^{\prime}$ simplesmente como uma componente de máximo em $H$. Em todos os casos, podemos supor sem perda da generalidade, que $C_{2}^{\prime}$ é da cor azul. Feito isso, estenda $C_{2}^{\prime}$ a uma componente azul maximal $C_{2}$ do grafo inteiro. 
Iremos nos referir de agora em diante a $C_{2}$ como a "componente azul". O que provaremos a seguir, depende apenas da maximalidade de $C_{2}$. Definiremos um grafo $H^{*}$ no qual queremos achar um emparelhamento máximo vermelho. Iremos obter algumas propriedades estruturais de $H^{*}$ e só então analisaremos alguns subcasos em função de como $C_{2}$ foi construída.

Comece tomando um emparelhamento máximo azul $M_{2}$ em $C_{2} \cap H$. O emparelhamento $M_{2}$ é conectado por definição, então podemos assumir que

$$
\left|M_{2}\right|=k_{2}=\left(\frac{1}{4}+\eta-\rho_{2}\right) n,
$$

para algum real positivo $\rho_{2}$. Usamos mais uma vez o lema 4.3, agora para selecionar as extremidades fortes das arestas de $M_{2}$. Denote o conjunto das extremidades fortes de $M_{2}$ por $U$ e seus demais vértices $\left(V\left(M_{2}\right) \backslash U\right)$ por $T$. Ademais, para $i=1,2,3$, sejam $U_{i}=U \cap V_{i}, T_{i}=T \cap V_{i}$ e $W_{i}=V_{i} \backslash V\left(M_{2}\right)$. Finalmente, para $1 \leq i, j \leq 3$, seja $M_{i j}$ o conjunto das arestas de $M_{2}$ que vão de $V_{i}$ para $V_{j}$ e denote $\left|M_{i j}\right|$ por $m_{i j}$.

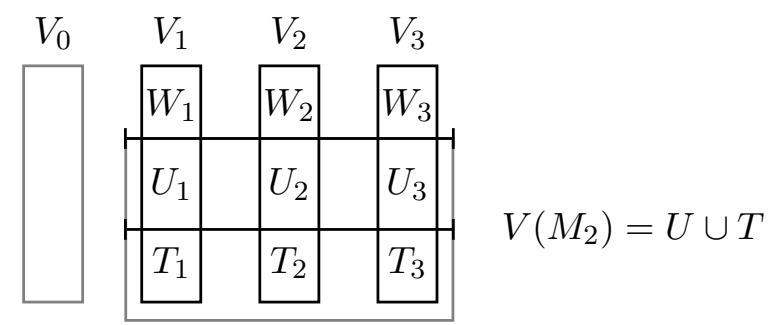

É trivial que, como o grafo $H$ não contém arestas internas a $V_{1}$ nem a $V_{3}$,

$$
\sum_{i=1}^{3}\left|T_{i}\right|=\sum_{i=1}^{3}\left|U_{i}\right|=m_{12}+m_{13}+m_{23}+m_{22}=k_{2} .
$$

O lema 4.3 e a maximalidade dos emparelhamentos verde e azul, $M_{1}$ e $M_{2}$, implicam, com a convenção de que cada aresta azul excepcional de cada vértice $u \in U$ para $W$ seja removida, que os seguintes subgrafos bipartidos de $H$, veja também a figura seguinte, possuem apenas arestas exclusivas da cor vermelha:

$$
\begin{gathered}
{\left[U_{1}, W_{2}\right],\left[U_{1}, W_{3}\right],\left[U_{2}, W_{1}\right],\left[U_{2}, W_{3}\right],\left[U_{3}, W_{1}\right],\left[U_{3}, W_{2}\right],} \\
{\left[U_{2}, W_{2}\right],\left[W_{1}, W_{2}\right],\left[W_{1}, W_{3}\right],\left[W_{2}, W_{3}\right] .}
\end{gathered}
$$

Seja $H^{*}$ o subgrafo de $H$ definido pela união dos subgrafos bipartidos acima. Note que todas as arestas de $H^{*}$ são vermelhas. A seguir, estabeleceremos inequações a fim de encontrar o tamanho do maior emparelhamento vermelho de $H^{*}$, que será então chamado de "emparelhamento vermelho" por simplicidade. É fácil checar que o emparelhamento vermelho é conectado. De fato, como $\left|W_{1}\right|+\left|W_{2}\right|+\left|W_{3}\right|=(1-\sqrt{\varepsilon}) n-k_{1}-2 k_{2}$, 


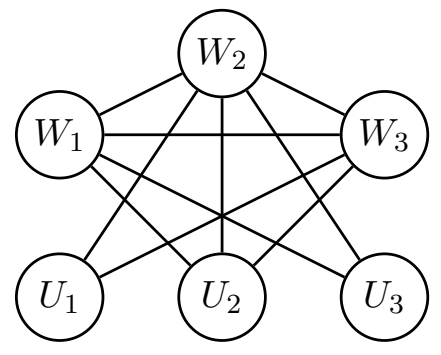

então para algum $i,\left|W_{i}\right|>4 \sqrt{\varepsilon} n$ (dado que $\varepsilon$ é pequeno). Então, o lema 4.5 nos garante até que todas as arestas de $H^{*}$ pertencem a uma mesma componente. Note que $H^{*}$ ainda pode ter, sendo generoso, $3 \sqrt{\varepsilon} n$ vértices isolados. Mas esses vértices podem ser removidos de $H^{*}$ sem prejudicar as cotas futuras. Por isso, assumimos que $H^{*}$ é conexo. Como fizemos para as outras cores, consideramos uma componente maximal vermelha de $G_{N}$ que contém $H^{*}$ e denotamos tal componente por $C_{3}$ ou como a "componente vermelha".

\section{Cortes críticos de $H^{*}$}

O objetivo agora é usar a fórmula de Tutte-Berge (lema 4.2) para encontrar um emparelhamento máximo em $H^{*}$. Contudo, note que

$$
\left|V\left(H^{*}\right)\right|=n-k_{1}-k_{2}=\left(\frac{1}{2}-2 \eta+\rho_{1}+\rho_{2}\right) n .
$$

Assim, mesmo um emparelhamento perfeito em $H^{*}$ pode não ser grande o bastante. Teríamos que cobrir mais $4 \eta n-\left(\rho_{1}+\rho_{2}\right) n$ vértices a fim de conseguir um emparelhamento vermelho de tamanho $\left(\frac{1}{4}+\eta\right) n$. A estratégia é tentar estender um emparelhamento máximo de $H^{*}$ a um emparelhamento do tamanho desejado em $G_{N}$.

Para começar, precisamos estimar o máximo de $c_{o}\left(H^{*} \backslash S\right)-|S|$ da fórmula de Tutte-Berge. Na verdade, estimaremos o valor máximo de $\operatorname{cr}(S)=c\left(H^{*} \backslash S\right)-|S|$, onde $c\left(H^{*} \backslash S\right)$ é o número de componente conexas de $H^{*} \backslash S$. Fazendo isto, não estamos perdendo muito, já que, como veremos, em $H^{*} \backslash S$ quase todas as componentes são pontos isolados e portanto são de ordem ímpar. Chamaremos um conjunto $S \subseteq V\left(H^{*}\right)$ de crítico se ele maximiza $\operatorname{cr}(S)$. Abaixo, calculamos o valor de $c r$ para uma coleção de cinco conjuntos específicos. Depois, mostraremos que, exceto pelo conjunto vazio, os conjuntos críticos possuem valor de cr próximo ao valor de cr desses conjuntos.

Afirmação 4.12. Seja $S_{1}=W_{1} \cup W_{2} \cup W_{3}, S_{2}=W_{2} \cup W_{3}, S_{3}=W_{1} \cup W_{2}, S_{4}=$ $U_{2} \cup U_{3} \cup W_{2} \cup W_{3}$ e $S_{5}=U_{1} \cup U_{2} \cup W_{1} \cup W_{2}$. Então, as componentes de $V\left(H^{*}\right) \backslash S_{i}$, para $i=1,4,5$, são respectivamente os vértices isolados de $U_{1} \cup U_{2} \cup U_{3}, U_{1} \cup W_{1} e$ $U_{3} \cup W_{3}$. As componentes de $V\left(H^{*}\right) \backslash S_{2}$ são os vértices isolados de $U_{1}$, possivelmente um componente própria de $W_{1} \cup U_{2} \cup U_{3}$ além de no máximo $6 \sqrt{\varepsilon} n$ vértices isolados 
neste mesmo conjunto. As componentes de $V\left(H^{*}\right) \backslash S_{3}$ são obtidas de maneira análoga às de $V\left(H^{*}\right) \backslash S_{2}$.

Ademais,

$$
\begin{array}{lcl}
\operatorname{cr}\left(S_{1}\right)=\left(\left|U_{1}\right|+\left|U_{2}\right|+\left|U_{3}\right|\right)-\left(\left|W_{1}\right|+\left|W_{2}\right|+\left|W_{3}\right|\right) & \leq\left(5 \eta-\left(\rho_{1}+3 \rho_{2}\right)\right) n \\
\operatorname{cr}\left(S_{2}\right) \leq & 6 \sqrt{\varepsilon} n+1+\left|U_{1}\right|-\left(\left|W_{2}\right|+\left|W_{3}\right|\right) & \leq\left(5 \eta-2\left(\rho_{1}+\rho_{2}\right)\right) n-\left|T_{1}\right| \\
\operatorname{cr}\left(S_{3}\right) \leq & 6 \sqrt{\varepsilon} n+1+\left|U_{3}\right|-\left(\left|W_{1}\right|+\left|W_{2}\right|\right) & \leq\left(5 \eta-\left(\gamma+\rho_{1}+2 \rho_{2}\right)\right) n-\left|T_{3}\right| \\
\operatorname{cr}\left(S_{4}\right)= & \left(\left|U_{1}\right|+\left|W_{1}\right|\right)-\left(\left|U_{2}\right|+\left|U_{3}\right|+\left|W_{2}\right|+\left|W_{3}\right|\right) & \leq\left(5 \eta-\left(3 \rho_{1}+\rho_{2}\right)\right) n-2\left|T_{1}\right| \\
\operatorname{cr}\left(S_{5}\right)= & \left(\left|U_{3}\right|+\left|W_{3}\right|\right)-\left(\left|U_{1}\right|+\left|U_{2}\right|+\left|W_{1}\right|+\left|W_{2}\right|\right) & \leq\left(5 \eta-\left(2 \gamma+\rho_{1}+2 \rho_{2}\right)\right) n-2\left|T_{3}\right|
\end{array}
$$

Em particular, $\operatorname{cr}\left(S_{i}\right) \leq 5 \eta n$ para todo $i$.

Prova. A afirmação sobre quais são as componentes de $V\left(H^{*}\right) \backslash S_{i}$ é imediata da definição de $H^{*}$. A cota superior para $\operatorname{cr}\left(S_{1}\right)$ vem do fato de que $\left|U_{1}\right|+\left|U_{2}\right|+\left|U_{3}\right|=k_{2}$ e $W_{1} \cup W_{2} \cup W_{3}=V\left(G_{N}\right) \backslash\left(V_{0} \cup V\left(M_{2}\right)\right)$. Assim, $\left|W_{1}\right|+\left|W_{2}\right|+\left|W_{3}\right|=N-k_{1}-2 k_{2}$. Observando isso, basta substituir os valores de $k_{1}$ e $k_{2}$ pelos valores indicados nas equações (4.1) e (4.5).

A cota para $\operatorname{cr}\left(S_{2}\right)$ segue de que

$$
\left|U_{1}\right|+\left|T_{1}\right|-\left(\left|W_{2}\right|+\left|W_{3}\right|\right)=\left|V_{0} \cup V_{1}\right|+\left|V\left(M_{2}\right)\right|-\left|V\left(G_{N}\right)\right|=2\left(k_{1}+k_{2}\right)-N .
$$

E, como antes, agora basta substituir os valores de $k_{1}$ e $k_{2}$. A cota para $\operatorname{cr}\left(S_{3}\right)$ é obtida de maneira análoga.

Como $\left|U_{1}\right|+\left|W_{1}\right|=k_{1}-\left|T_{1}\right|$ e $\left|U_{2}\right|+\left|U_{3}\right|+\left|W_{2}\right|+\left|W_{3}\right|=|U|+|W|-\left(\left|U_{1}\right|+\left|W_{1}\right|\right)$, conseguimos a cota para $\operatorname{cr}\left(S_{4}\right)$ da maneira a seguir.

$$
\begin{aligned}
\left(\left|U_{3}\right|+\left|W_{3}\right|\right)-\left(\left|U_{1}\right|+\left|U_{2}\right|+\left|W_{1}\right|+\left|W_{2}\right|\right) & =2\left(k_{1}-\left|T_{1}\right|\right)-(|U|+|W|) \\
& =2\left(k_{1}-\left|T_{1}\right|\right)-\left(N-k_{1}-k_{2}\right) \\
& =3 k_{1}+k_{2}-N-2\left|T_{1}\right| .
\end{aligned}
$$

Novamente, basta substituir os valores de $k_{1}$ e $k_{2}$. A cota para $\operatorname{cr}\left(S_{5}\right)$ segue de maneira análoga.

Afirmação 4.13. Para todo $S \subseteq V\left(H^{*}\right)$, vale que

$$
\operatorname{cr}(S) \leq \max _{0 \leq i \leq 5}\left\{\operatorname{cr}\left(S_{i}\right)\right\}+24 \sqrt{\varepsilon} n,
$$

onde $S_{0}=\emptyset\left(\right.$ e então $\left.\operatorname{cr}\left(S_{0}\right) \leq 1\right)$.

Prova. Seja $S$ um subconjunto qualquer de $V\left(H^{*}\right)$. Particione $S$ em seis partes: $S \cap U_{i}$, $S \cap W_{i}$, para $i=1,2,3$. Seja $S^{*}=S \cup M$, onde $M$ é a união dos conjuntos $U_{i}$ 's e $W_{i}$ 's que satisfazem $\left|U_{i} \backslash S\right|<2 \sqrt{\varepsilon} n$ ou $\left|W_{i} \backslash S\right|<2 \sqrt{\varepsilon} n$. Então, temos que

$$
\left|S^{*}\right| \leq|S|+12 \sqrt{\varepsilon} n \text {. }
$$


Afirmação 4.14. Vale que $\operatorname{cr}\left(S^{*}\right)<\max _{0 \leq i \leq 5}\left\{\operatorname{cr}\left(S_{i}\right)\right\}$.

Provemos primeiro a afirmação. Chame o conjunto $U_{i}$ (resp., $W_{i}$ ) de cheio, se $S^{*} \cap U_{i}=U_{i}$ (resp., $S^{*} \cap W_{i}=W_{i}$ ). Observe que, se $i \neq j$ ou $i=j=2$ e nem $U_{i}$ nem $W_{j}$ é cheio, então $\left|U_{i} \backslash S^{*}\right| \geq 2 \sqrt{\varepsilon} n$ e $\left|W_{j} \backslash S^{*}\right| \geq 2 \sqrt{\varepsilon} n$ (de acordo com a definição de $S^{*}$ a partir de $S$ ). Então, pelo lema 4.5, o grafo bipartido $\left[U_{i} \backslash S^{*}, W_{j} \backslash S^{*}\right]$ é conexo. O mesmo argumento mostra que $\left[W_{i} \backslash S^{*}, W_{j} \backslash S^{*}\right]$ é conexo no caso em que $i \neq j$ e ambos $W_{i}$ e $W_{j}$ não são cheios. Este argumento mostra que $H^{*} \backslash S^{*}$ possui no máximo uma componente não trivial, ou seja, todas as demais componentes são pontos isolados. É óbvio que remover vértices de $S^{*}$ pertencentes a componentes não cheias não reduz o número de componentes de $H^{*} \backslash S^{*}$. Assim, podemos assumir que os conjuntos $W_{i}$ e $U_{i}$ ou são cheios ou são vazios (i.e., $U_{i} \cap S^{*}$ e $W_{i} \cap S^{*}$ são vazios). Isto se reduz a checar o valor de $\operatorname{cr}(S)$ para o grafo ponderado formado por seis vértices, o esqueleto de $H^{*}$, definido sobre o conjunto de vértices $u_{i}, w_{i}$ para $1 \leq i \leq 3$ e arestas $\left\{u_{i}, w_{j}\right\},\left\{w_{i}, w_{j}\right\}$ para $1 \leq i<j \leq 3$ e $\left\{u_{2}, w_{2}\right\}$, com pesos $\left|U_{i}\right|$ nos vértices $u_{i}$ e $\left|W_{i}\right|$ nos vértices $w_{i}$. Um momento de reflexão mostra que os valores não negativos de $\operatorname{cr}(S)$ são dados apenas pelos cinco conjuntos $S_{i}$ da afirmação 4.12 e por $S_{0}$. Isto completa a prova da afirmação.

Observe que, para $X \subseteq X^{*}$,

$$
c(G \backslash X)-|X| \leq c\left(G \backslash X^{*}\right)-\left|X^{*}\right|+2\left(\left|X^{*}\right|-|X|\right) .
$$

Usando esta observação, a afirmação 4.14 e a equação (4.7), temos que

$$
\begin{aligned}
\operatorname{cr}(S) & =c\left(H^{*} \backslash S\right)-|S| \leq c\left(H^{*} \backslash S^{*}\right)-\left|S^{*}\right|+2\left(\left|S^{*}\right|-|S|\right)= \\
& =\operatorname{cr}\left(S^{*}\right)+2\left(\left|S^{*}\right|-|S|\right) \leq \max _{0 \leq i \leq 5}\left\{\operatorname{cr}\left(S_{i}\right)\right\}+2 \times 12 \sqrt{\varepsilon} n,
\end{aligned}
$$

para qualquer $S \subseteq V\left(H^{*}\right)$, finalizando a prova do lema.

Do lema 4.2 e da afirmação 4.13, segue o seguinte corolário.

Corolário 4.15. Vale que $2 \nu\left(H^{*}\right) \geq\left|V\left(H^{*}\right)\right|-\max _{0 \leq i \leq 5}\left\{\operatorname{cr}\left(S_{i}\right)\right\}-24 \sqrt{\varepsilon} n$.

Agora, precisamos saber qual cr $\left(S_{i}\right)$ é máximo, mas é claro que isto depende de vários fatores. Um caso simples é se $\rho_{1}+\rho_{2} \geq 5 \eta$. Neste caso, $\operatorname{cr}\left(S_{i}\right)$ é negativo para todo $1 \leq i \leq 5$ e portanto $\operatorname{cr}\left(S_{0}\right)$ é máximo. Daí, o corolário anterior assegura que $H^{*}$ possui um emparelhamento vermelho cobrindo pelo menos

$$
\left|V\left(H^{*}\right)\right|-1-24 \sqrt{\varepsilon} n=\left(\frac{1}{2}-2 \eta+\rho_{1}+\rho_{2}-24 \sqrt{\varepsilon}\right) n-1>\left(\frac{1}{2}+2 \eta\right) n
$$

vértices. Ou seja, achamos um emparelhamento vermelho de tamanho pelo menos $\left(\frac{1}{4}+\eta\right) n$. Então, podemos assumir que

$$
\rho_{1}+\rho_{2}<5 \eta
$$


Agora, queremos encontrar um emparelhamento em $\left[V\left(H^{*}\right), V\left(G_{N}\right) \backslash V\left(H^{*}\right)\right]$ que estenda o emparelhamento vermelho a um emparelhamento do tamanho desejado. Uma maneira de tentar fazer isso é usando o fato a seguir.

Afirmação 4.16. Suponha que existe $Z$, subconjunto de $U_{i}$ ou subconjunto de $W_{i}$, tal que $|Z|=\Delta$ e que existe um conjunto de $\Delta$ arestas vermelhas independentes em $\left[Z, V\left(G_{N}\right) \backslash V\left(H^{*}\right)\right]$. Se todo $\operatorname{cr}\left(S_{i}\right), 1 \leq i \leq 5$, é negativo ou não aumenta no grafo $H^{*} \backslash$ $Z$ então podemos achar o emparelhamento do tamanho desejado. Veja, por exemplo, que se $\left|T_{1}\right| \geq 2 \Delta$, então os valores de $\operatorname{cr}\left(S_{i}\right)$, para $i=2$, 4 , em relação ao grafo $H^{*} \backslash Z$ são negativos. $E$, se $\gamma n \geq 2 \Delta$ ou $\left|T_{3}\right| \geq 2 \Delta$, então os valores de $\operatorname{cr}\left(S_{i}\right)$, para $i=3,5$, em relação ao grafo $H^{*} \backslash Z$ são negativos.

Prova. Como originalmente $\operatorname{cr}\left(S_{i}\right) \leq 5 \eta n$, para todo $i$, e o valor $\operatorname{de} \operatorname{cr}\left(S_{i}\right)$ não aumenta quando o grafo $H^{*} \backslash Z$ é tomado como universo, então, pela formula de Tutte-Berge,

$2 \nu\left(H^{*} \backslash Z\right) \geq\left|V\left(H^{*} \backslash Z\right)\right|-5 \eta n-24 \sqrt{\varepsilon} n \geq\left(\frac{1}{2}-2 \eta-10 \eta\right) n-5 \eta n-24 \sqrt{\varepsilon} n>\left(\frac{1}{2}-18 \eta\right) n$.

Adicionando as $\Delta=10 \eta n$ arestas de $\left[Z, V\left(G_{N}\right) \backslash V\left(H^{*}\right)\right]$, ficamos então com um emparelhamento com $\left(\frac{1}{2}+2 \eta\right) n$ vértices. As demais afirmações são triviais a partir da inspeção das fórmulas da afirmação 4.12 .

Feito tudo isto, podemos começar a analisar os subcasos do caso 2. Aqui, precisamos lembrar como $C_{2}$ foi construída. Veja que, pela equação (4.8), em particular, podemos assumir que $\rho_{1}<5 \eta$. Assim, as cotas em (4.4) são válidas. Logo, precisamos considerar apenas os casos em que $\left|V_{3}\right|<4 \sqrt{\varepsilon} n$ ou $C_{2}$ foi obtida através uma aplicação do lema 4.9 ao grafo tripartido das arestas vermelhas de $V_{1} \cup V_{2} \cup V_{3}$. Sendo assim, a análise de casos feita a seguir cobre todas as possíveis situações.

\section{Subcasos do Caso 2}

Subcaso 2.1: $\left|U_{3}\right|+\left|W_{3}\right| \leq 2 \Delta$ ou $\left|V_{3}\right| \leq 9 \Delta$.

Se $\left|T_{1}\right| \leq \Delta$, então usando (4.8) temos

$$
\begin{aligned}
\left|T_{2} \cup T_{3}\right| & \geq k_{2}-\left|T_{1}\right| \geq\left(\frac{1}{4}+\eta-\rho_{2}\right) n-\Delta \geq\left(\frac{1}{4}-14 \eta\right) n ; \\
\left|U_{1} \cup W_{1}\right| & =\left|V_{1}\right|-\left|T_{1}\right| \geq\left(\frac{1}{4}+\eta-\rho_{1}\right) n-\Delta \geq\left(\frac{1}{4}-14 \eta\right) n ; \\
\left|U_{2} \cup W_{2}\right| & =\left|V_{2}\right|-\left|T_{2}\right|=\left(N-2 k_{1}-\left|V_{3}\right|\right)-\left|T_{2}\right| \geq \\
& \geq N-\left(2 k_{1}+\left|T_{3}\right|+2 \Delta+\left|T_{2}\right|\right) \geq \\
& \geq N-\left(2 k_{1}+k_{2}+2 \Delta\right) \geq\left(\frac{1}{4}-24 \eta\right) n ; \\
\left|U_{1} \cup U_{2}\right| & \leq k_{2}=\left(\frac{1}{4}+\eta-\rho_{2}\right) n .
\end{aligned}
$$


Logo, achamos uma $\mathrm{WEC}_{2} \operatorname{com} C=T_{2} \cup T_{3}, A=U_{1} \cup W_{1}, B=U_{2} \cup W_{2}$ e $Q=U_{1} \cup U_{2}$, e cores vermelha e azul $(\operatorname{com} \beta=24 \eta)$. Veja que, para obter a cota inferior para $\left|U_{2} \cup W_{2}\right|$, usamos a condição $\left|U_{3}\right|+\left|W_{3}\right| \leq 2 \Delta$ para concluir que $\left|V_{3}\right| \leq\left|T_{3}\right|+2 \Delta$; uma cota semelhante, mas com erro um pouco maior, poderia ser obtida diretamente no caso em que sabemos apenas que $\left|V_{3}\right| \leq 9 \Delta$.

Agora, $\left|T_{1}\right|>\Delta$. Veja também que, $\left|T_{3}\right|>2 \Delta$ ou $\gamma n>2 \Delta$. De fato, se $\left|T_{3}\right| \leq 2 \Delta$, então da equação (4.3) segue que

$$
\left(\frac{1}{4}+2 \eta-\gamma-\sqrt{\varepsilon}\right) n \leq\left|V_{3}\right|=\left|T_{3}\right|+\left|U_{3}\right|+\left|W_{3}\right| \leq 9 \Delta,
$$

que vale tanto no caso em que $\left|U_{3}\right|+\left|W_{3}\right| \leq 2 \Delta$, como no caso em que temos a cota direta $\left|V_{3}\right| \leq 9 \Delta$. Isto implica $\gamma n \geq\left(\frac{1}{4}+2 \eta\right) n-9 \Delta-\sqrt{\varepsilon} n>2 \Delta$ (dado que $\varepsilon$ e $\eta$ são pequenos o suficiente). Assim, a afirmação 4.16 nos garante que $\operatorname{cr}\left(S_{i}\right)$, para $i=2,3,4,5$, continua negativo mesmo após a remoção de $\Delta$ vértices de um dos conjuntos $U_{i}$ ou $W_{i}$. Teremos então que nos preocupar apenas $\operatorname{com} \operatorname{cr}\left(S_{1}\right)$.

Note que, se removermos um conjunto de $\Delta$ vértices de $U_{2}$, então o valor $\operatorname{de} \operatorname{cr}\left(S_{1}\right)$ diminui. Assim, a afirmação 4.16 nos garante que, se existem $\Delta$ arestas vermelhas independentes em $\left[U_{2}, V(G) \backslash V\left(H^{*}\right)\right]$, conseguimos encontrar um emparelhamento vermelho conectado cobrindo pelo menos $\left(\frac{1}{2}+2 \eta\right) n$ vértices e a prova acaba aqui. Logo, podemos assumir que não conseguimos encontrar as $\Delta$ arestas independentes. Em particular, as arestas vermelhas de $\left[U_{2}, T_{1}\right]$ e $\left[U_{2}, T_{2}\right]$ podem ser cobertas por no máximo $\Delta$ vértices, de modo que se removermos tais vértices eliminamos todas as arestas vermelhas desses grafos. Neste caso, dizemos que os grafos bipartidos $\left[U_{2}, T_{1}\right]$ e $\left[U_{2}, T_{2}\right]$ são quase exclusivos da cor azul. Do mesmo modo, $\left[U_{1}, T_{2}\right]$ é quase exclusivo azul e $\left[U_{2}, V_{0}\right],\left[U_{1}, V_{0}\right]$ são quase exclusivos das cores azul ou verde.

A seguir, olhamos para $I=\left[U_{1}, U_{2}\right]$. Assuma primeiro que $I$ possui um conjunto $F$ de $\Delta / 2$ arestas independentes vermelhas. Faremos algo um pouco diferente aqui. Se existem $\Delta$ arestas vermelhas independentes em $\left[W_{1}, V(G) \backslash V\left(H^{*}\right)\right]$, então removemos, além dos $\Delta$ vértices de $W_{1}$, os vértices de $V(F) \subseteq U_{1} \cup U_{2}$. Isso faz com que $\operatorname{cr}\left(S_{1}\right)$ não aumente e ainda assim os valores de $\operatorname{cr}\left(S_{i}\right)$, para $i \neq 1$, continuam negativos. Dessa forma conseguimos encontrar o emparelhamento desejado. Podemos fazer o mesmo, se existem $\Delta$ arestas vermelhas independentes em $\left[W_{2}, V(G) \backslash V\left(H^{*}\right)\right]$. Podemos assumir então que, em particular, os grafos $\left[W_{1}, T_{2}\right],\left[W_{1}, T_{3}\right],\left[W_{2}, T_{1}\right],\left[W_{2}, T_{2}\right],\left[W_{2}, T_{3}\right]$ são quase exclusivos da cor azul, e que $\left[W_{1}, V_{0}\right],\left[W_{2}, V_{0}\right]$ são quase exclusivos das cores azul ou verde. Com isso, concluímos também que as arestas azuis de $\left[W_{1}, V_{0}\right] \cup$ $\left[W_{2}, V_{0}\right]$ pertencem à componente $C_{2}$, ou seja, estão na mesma componente de $M_{2}$. Assim, $\left(\frac{1}{4}+\eta\right)-k_{2}=\rho_{2} n<\Delta$ arestas independentes azuis em $\left[W_{1}, V_{0}\right] \cup\left[W_{2}, V_{0}\right]$ seriam suficiente para estender $M_{2}$ a um emparelhamento azul conectado de tamanho $\left(\frac{1}{4}+\eta\right) n$, e terminar a prova. Então $\left[W_{1}, V_{0}\right],\left[W_{2}, V_{0}\right]$ são quase exclusivos da cor verde. Também $\left[U_{1}, V_{0}\right],\left[U_{2}, V_{0}\right]$ são quase exclusivos da cor verde, caso contrário $M_{2}$ poderia ser estendido através das arestas azuis de $\left[W_{2}, T_{1}\right],\left[W_{1}, T_{2}\right],\left[W_{2}, T_{2}\right],\left[W_{1}, T_{3}\right]$, $\left[W_{2}, T_{3}\right]$. É claro que removendo no máximo $4 \Delta$ vértices de cada conjunto envolvido (o 
que eventualmente deixará alguns desses conjuntos vazios), conseguimos obter grafos bipartidos com arestas exclusivas de uma ou duas cores apropriadas, ao invés de apenas quase exclusivos. Então, conseguimos uma $\mathrm{WEC}_{2} \operatorname{com} A=U_{1} \cup U_{2}, B=W_{1} \cup W_{2}$, $C=V_{0}, Q=U_{1} \cup W_{1}$, nas cores vermelha e verde, e estamos satisfeitos.

Finalmente, se $I$ for quase exclusivo da cor azul, o papel das cores azul e vermelha poderia ser invertido. Mais precisamente, note que removendo no máximo $4 \Delta$ vértices de cada $U_{i}, T_{i}$ os grafos bipartidos quase exclusivos da cor azul,

$$
\left[T_{1}, U_{2}\right],\left[T_{2}, U_{1}\right],\left[T_{3}, U_{1}\right],\left[T_{3}, U_{2}\right],\left[T_{2}, U_{2}\right],
$$

se tornariam exclusivos da cor azul. Denotando por $H^{* *}$ a união destes grafos, notamos que

$$
\left|V\left(H^{* *}\right)\right| \geq 2 k_{2}-24 \Delta \geq\left(\frac{1}{2}-252 \eta\right) n .
$$

Os conjuntos críticos de $H^{* *}$ poderiam ser analisados da mesma maneira que os de $H^{*}$ a fim de formalizarmos precisamente o argumento do parágrafo a seguir. Mas esta análise será omitida.

Escolhendo $\Delta^{*}$ grande, digamos $\Delta^{*}=600 \eta$, ou o emparelhamento azul de $H^{* *}$ pode ser aumentado em $\Delta^{*}$ para um emparelhamento azul conectado de tamanho $\left(\frac{1}{4}+\eta\right) n$, ou $\left[T_{2}, W_{1}\right],\left[T_{2}, W_{2}\right],\left[T_{1}, W_{2}\right],\left[T_{3}, W_{1}\right],\left[T_{3}, W_{2}\right]$ são quase exclusivos da cor vermelha e isto implica que $\left[V_{0}, T_{1}\right],\left[V_{0}, T_{2}\right],\left[V_{0}, T_{3}\right]$ são quase exclusivos da cor verde. Neste caso encontramos uma coloração $\mathrm{WEC}_{2} \operatorname{com} A=U_{1} \cup U_{2}, B=T_{1} \cup T_{2} \cup T_{3}, C=V_{0}$, $Q=U_{1} \cup T_{1}$, e cores azul e vermelha.

Para os próximos subcasos, defina $F=V_{0} \backslash V\left(C_{2}\right)$ e $P=V_{0} \backslash V\left(C_{3}\right)$.

Subcaso 2.2: $C_{2}$ cobre todos exceto no máximo $4 \Delta$ vértices de $H$.

Podemos assumir que $\left|U_{3}\right|+\left|W_{3}\right|>2 \Delta$ e $\left|V_{3}\right|>9 \Delta$, caso contrário o subcaso anterior se aplica. Como $9 \Delta<\left|V_{3}\right| \leq\left(\frac{1}{4}+2 \eta-\gamma\right) n$, temos que $\gamma n<\left(\frac{1}{4}+2 \eta\right) n-9 \Delta$. Como $C_{1}$ é uma componente de ordem máxima, $n-|F|-4 \Delta<\left|C_{2}\right| \leq\left|C_{1}\right|=\left(\frac{3}{4}-\right.$ $\eta-6 \sqrt{\varepsilon}+\gamma) n$ e temos

$$
|F|>\left(\frac{1}{4}+2 \eta-\gamma\right) n-4 \Delta>2 \Delta
$$

(dado que $\eta, \varepsilon$ são pequenos o suficiente). Note que (4.9) implica $\left|T_{3}\right| \leq \Delta+\sqrt{\varepsilon} n$ caso contrário, como $F$ pertence a $C_{3}$ (componente vermelha) devido às arestas de $\left[F, U_{3} \cup\right.$ $\left.W_{3}\right]$, usando o lema 4.5 para as arestas vermelhas de $\left[F, T_{3}\right]$ conseguimos aumentar o emparelhamento vermelho em $\Delta$. Disto concluímos que

$$
\left|U_{3}\right|+\left|W_{3}\right|>8 \Delta-\sqrt{\varepsilon} n .
$$

Se $\left|T_{1}\right| \leq \Delta$, então encontramos uma $\mathrm{WEC}_{2}$ com

$$
A=U_{1} \cup W_{1}, B=U_{2} \cup U_{3} \cup W_{2} \cup W_{3}, C=T_{2}, Q=U_{1} \cup U_{2} \cup U_{3}
$$


e terminamos a prova usando o lema 4.11.

Portanto, podemos assumir que $\left|T_{1}\right|>\Delta$. Observe que (4.10) e a escolha de $C_{2}$ implicam que, ou $I:=V\left(C_{2}\right) \cap U_{3}$, ou $J:=V\left(C_{2}\right) \cap W_{3}$ tem ordem pelo menos $2 \Delta-$ $\frac{1}{2} \sqrt{\varepsilon} n$. Lembre-se que as arestas de $[F, I],[F, J]$ devem ser vermelhas. Suponha primeiro que $|I| \geq 2 \Delta-\frac{1}{2} \sqrt{\varepsilon} n$. Então temos $\Delta$ arestas vermelhas independentes em $[F, I]$, que podemos usar para estender o emparelhamento vermelho a um do tamanho desejado usando a afirmação 4.16 , já que apenas $\operatorname{cr}\left(S_{4}\right)$ poderia aumentar após a remoção das extremidades dessas arestas, mas $\operatorname{cr}\left(S_{4}\right)$ é negativo $\left(\left|T_{1}\right|>\Delta\right)$. Logo, podemos assumir $|I|<2 \Delta-\frac{1}{2} \sqrt{\varepsilon} n$ e $|J| \geq 2 \Delta-\frac{1}{2} \sqrt{\varepsilon} n$.

Agora, uma versão mais simples do argumento usado no subcaso 2.1 será aplicado. Podemos estender o emparelhamento vermelho adicionando $\frac{\Delta}{2}$ arestas independentes de $\left[U_{1}, V(G) \backslash V\left(H^{*}\right)\right]$ e simultaneamente $\frac{\Delta}{2}$ arestas independentes de $[F, J]$. A remoção das extremidades dessas arestas não afeta o valor de nenhum $\operatorname{dos} \operatorname{cr}\left(S_{i}\right)$, pois estamos decrementando o mesmo valor de $\left|U_{1}\right|$ e $\left|W_{3}\right|$ ao mesmo tempo. Assim, podemos alcançar o tamanho de emparelhamento desejado. Concluímos que $\left[U_{1}, T_{2}\right]$ é quase exclusivo da cor azul e $\left[U_{1}, V_{0}\right],\left[U_{1}, T_{1}\right]$ são quase exclusivos das cores azul e verde. O mesmo argumento pode ser aplicado para mostrar $\left[U_{2}, T_{1}\right],\left[U_{2}, T_{2}\right]$ são quase exclusivos da cor azul e $\left[U_{2}, V_{0}\right]$ é quase exclusivo das cores azul e verde. Contudo, aqui temos que tomar um pouco mais de cuidado já que decrementar simultaneamente $U_{2}$ e $W_{3}$ pode aumentar os valores de $\operatorname{cr}\left(S_{2}\right)$ e $\operatorname{cr}\left(S_{4}\right)$, mas não há problema pois $\left|T_{1}\right|>\Delta$. Se $|P| \geq 2 \Delta$, então, pelo lema 4.5, o subgrafo azul $[P, J]$ tem um emparelhamento de tamanho $\Delta / 2$ que estende $M_{2}$ ao tamanho que precisamos. Portanto, podemos assumir que $|P|<2 \Delta$. Se os subgrafos vermelhos $\left[T_{1}, V_{0} \backslash P\right]$ e $\left[T_{2}, V_{0} \backslash P\right]$ possuem $\Delta$ arestas independentes, então podemos estender o emparelhamento vermelho a um emparelhamento vermelho conectado do tamanho adequado. Caso contrário, estes grafos são quase exclusivos das cores azul e verde e então temos uma $\mathrm{WEC}_{2} \mathrm{com}$

$$
A=T_{1} \cup T_{2}, B=U_{1} \cup U_{2}, C=V_{0}, Q=T_{1} \cup U_{1},
$$

o que finaliza este subcaso.

Subcaso 2.3: $C_{2}$ cobre $V_{2} \cup V_{3}$ (e parte de $V_{1}$ ).

Seja $Y=V_{1} \backslash V\left(C_{2}\right)$. Claramente, $Y \subseteq W_{1}$, pois $C_{2}$ contém $U_{1} \cup T_{1}$ por definição. Seja $C_{3}^{\prime}$ uma extensão maximal da componente vermelha que cobre $V_{2} \cup V_{3}$ e $X=V_{1} \backslash V\left(C_{3}^{\prime}\right)$. Também é claro que $X \subseteq T_{1}$. Podemos assumir que $|Y|>4 \Delta$, caso contrário teríamos aplicado o caso 2.2 , e também que $|X|>4 \Delta$, já que definimos $C_{2}$ como a maior das duas componentes cobrindo parte de $V_{1}$. Observe que, se $v \in V_{0} \backslash\left(V\left(C_{2}\right) \cup V\left(C_{3}^{\prime}\right)\right)$, então nenhuma das cores poderia ser atribuída a uma aresta $v t$ com $t \in V_{3}$. Assim, $\left|V_{3}\right| \leq \sqrt{\varepsilon} n$ e isto já foi tratado no caso 2.1. Logo, $C_{2} \cup C_{3}^{\prime}$ cobre todos os vértices de $V_{0}$.

Suponha primeiro que $|F| \geq 2 \Delta$. Aqui, um argumento idêntico do caso anterior funciona. Como antes, $\left|T_{3}\right| \leq \Delta+\sqrt{\varepsilon} n$, caso contrário, as arestas de $\left[F, T_{3}\right]$ seriam 
vermelhas e, pelo lema 4.5, poderíamos aumentar o emparelhamento vermelho em $\Delta$. Isto implica que $\left|U_{3}\right|+\left|W_{3}\right|>5 \Delta$. Se $\left|U_{3}\right| \geq 2 \Delta$, então podemos aumentar o emparelhamento vermelho já que todas as arestas de $\left[U_{3}, F\right]$ são vermelhas e que apenas $\operatorname{cr}\left(S_{4}\right)$ poderia aumentar ao removermos vértices de $V_{3}$, mas $\operatorname{cr}\left(S_{4}\right)$ é negativo (pois $\left|T_{1}\right|>\Delta$ ). Se $\left|W_{3}\right| \geq \Delta$, então podemos aumentar o emparelhamento vermelho adicionando a ele $\Delta / 2$ arestas de $\left[U_{1}, V(G) \backslash V\left(H^{*}\right)\right]$ e simultaneamente $\Delta / 2$ arestas de $\left[F, W_{3}\right]$. Isto não afeta nenhum $\operatorname{dos} \operatorname{cr}\left(S_{i}\right)$ (pois estamos decrementando ao mesmo tempo o valor $\left|U_{1}\right|$ e $\left.\left|W_{3}\right|\right)$. Concluímos então que $\left[U_{1}, T_{2}\right]$ é quase exclusivo da cor azul, e $\left[U_{1}, V_{0}\right],\left[U_{1}, T_{1}\right]$ são quase exclusivos das cores azul e verde. O mesmo argumento pode ser aplicado para provar $\left[U_{2}, T_{1}\right],\left[U_{2}, T_{2}\right]$ são quase exclusivos em azul e $\left[U_{2}, V_{0}\right]$ é quase exclusivo em azul e verde. Se $|P| \geq 2 \Delta$ então pelo lema 4.5 o subgrafo azul $\left[P, W_{3}\right]$ possui um emparelhamento de tamanho $\Delta / 2$ que podemos usar para estender $M_{2}$ a um emparelhamento como desejamos. Logo, podemos assumir que $|P|<2 \Delta$. Se os subgrafos vermelhos $\left[T_{1}, V_{0} \backslash P\right]$ e $\left[T_{2}, V_{0} \backslash P\right]$ possuem $\Delta$ arestas independentes, então podemos estender o emparelhamento vermelho a um emparelhamento vermelho. Caso contrário, estes grafos são quase exclusivos em azul e verde e temos uma $\mathrm{WEC}_{2} \mathrm{com}$

$$
A=T_{1} \cup T_{2}, B=U_{1} \cup U_{2}, C=V_{0}, Q=T_{1} \cup U_{1} .
$$

Resta o caso em que $|F|<2 \Delta$. Isso nos dá que $\gamma$ é grande. De fato, usando que $|F|+|Y|=N-\left|C_{2}\right| \geq N-\left|C_{1}\right|=\left|V_{3}\right|$, temos que

$$
\begin{aligned}
2 \Delta+\left(\frac{1}{4}+\eta-\rho_{1}\right) n-4 \Delta & \geq|F|+\left|V_{1}\right|-|X| \geq|F|+|Y| \geq\left|V_{3}\right| \\
& \geq\left(\frac{1}{4}+2 \eta-\gamma-\sqrt{\varepsilon}\right) n,
\end{aligned}
$$

ou seja, $\gamma n \geq 2 \Delta+\left(\eta+\rho_{1}-\sqrt{\varepsilon}\right) n>2 \Delta$. Também sabemos que $\left|T_{1}\right| \geq|X| \geq 4 \Delta$, então podemos usar a afirmação 4.16 novamente e seguir o mesmo argumento do subcaso 2.1. Ou seja, os grafos $\left[U_{i}, T_{j}\right]$, para $1 \leq i \neq j \leq 3$ e $i=j=2$, são quase exclusivos da cor azul. Então tomamos $Z_{i j}=\left[U_{i}, U_{j}\right]$, para $1 \leq i<j \leq 3$. A hipótese de que $Z_{i j}$ possui $\Delta$ arestas independentes vermelhas implica que $\left[W_{i}, T_{j}\right]$ são quase exclusivos da cor azul para $1 \leq i<j \leq 3$ e $i=j=2$. Contudo, as arestas de $\left[Y, T_{2} \cup T_{3}\right]$ são vermelhas e, como $|Y| \geq 4 \Delta$, isto é possível somente se $\left|T_{2} \cup T_{3}\right| \leq 2 \Delta$. Isto implica que $\left|T_{1}\right|=k_{2}-\left|T_{2} \cup T_{3}\right| \geq\left(\frac{1}{4}+\eta-\rho_{2}\right) n-2 \Delta$ e então $\left|U_{1} \cup W_{1}\right|=k_{1}-\left|T_{1}\right| \leq 2 \Delta+\left(\rho_{2}-\rho_{1}\right) n$. Contudo, pela definição de $\Delta$ e pela equação (4.8),

$$
40 \eta n=4 \Delta \leq|Y| \leq\left|U_{1} \cup W_{1}\right| \leq 2 \Delta+\left(\rho_{2}-\rho_{1}\right) n \leq 20 \eta n+\rho_{2} \leq 25 \eta n,
$$

o que é uma contradição.

Então $Z_{i j}$ é quase exclusivo da cor azul (para todos os índices válidos) e podemos usar o mesmo argumento do último parágrafo do caso 2.1 . 
Subcaso 2.4: $C_{2}$ cobre $V_{1} \cup V_{2}$ (e parte de $V_{3}$ ).

Seja $Y=V_{3} \backslash V\left(C_{2}\right)$, claramente $Y \subseteq W_{3}$. Defina $C_{3}^{\prime}$ de modo análogo ao caso anterior e seja $X=V_{3} \backslash V\left(C_{3}^{\prime}\right)$, claramente $X \subseteq T_{3}$ e $|X|,|Y| \geq 4 \Delta$, caso contrário estamos no caso 2.2. Segue também que $X=T_{3}$ e $Y=W_{3}$. É fácil ver que os conjuntos $\left(C_{2} \backslash C_{3}^{\prime}\right) \cap V_{0},\left(C_{3}^{\prime} \backslash C_{2}\right) \cap V_{0}$ são vazios. Agora, $X_{1}=V_{0} \backslash V\left(C_{2}\right), X_{2}=$ $V\left(C_{1}\right) \cap V\left(C_{2}\right), X_{3}=X$ e $X_{4}=Y$ define uma 4-partição de $V\left(G_{N}\right)$. Ademais, é fácil ver que $\left|X_{i}\right|>10 \eta n$ para todo $1 \leq i \leq 4$. De fato, $\left|X_{1}\right| \leq 10 \eta n$ contrariaria a escolha de $C_{1},\left|X_{2}\right|>10 \eta n$ é trivial e $\left|X_{3}\right|,\left|X_{4}\right| \geq 4 \Delta>10 \eta n$. Então ou o subcaso 1.1, ou o subcaso 1.2 pode ser aplicado. Isto encerra o caso 2.4 .

Subcaso 2.5: A observação final para o caso 2 é que o subcaso em que $C_{2}$ cobre $V_{1} \cup V_{3}$ (e parte de $V_{2}$ ), na verdade, não acontece. De fato, neste caso nenhuma cor poderia ser atribuída para as arestas de $\left[\left(V_{2} \cap C_{2}\right) \backslash C_{3}^{\prime},\left(V_{2} \cap C_{3}^{\prime}\right) \backslash C_{2}\right]$ (aqui $C_{3}^{\prime}$ também é definida de modo análogo aos casos anteriores).

Isto termina a prova do lema 4.1.

\subsubsection{Prova do lema 4.11: reduzindo colorações extremais fracas}

Prova. Sejam $\eta, \varepsilon>0$ tais que $\varepsilon$ é pequeno em relação a $\eta$ e seja $G_{N}$ um grafo como no enunciado. Sejam $A, B, C, Q \subset V\left(G_{N}\right)$, como na definição da $\mathrm{WEC}_{2}$ e seja $D=V\left(G_{N}\right) \backslash(A \cup B \cup C)$. Usaremos as cores vermelha e azul para as cores $i, j$ na definição de $\mathrm{WEC}_{2}$.

Por conveniência, denote $n / 4$ por $m$. Removendo vértices de $A, B, C$, podemos assumir sem perda da generalidade que $|A|,|B|,|C|=(1-\beta) m$ e que $|D| \leq(1+3 \beta) m$. Iremos manter um parâmetro $p$ de modo que $(1-p) m$ seja uma cota inferior para a ordem dos conjuntos $A, B, C, D$. Apesar de que em cada passo apenas as cotas inferiores de um ou dois dos conjuntos precisariam ser alteradas, nós usamos um limite inferior comum para simplificar os cálculos. Inicialmente $p=\beta$. Como $|Q|<(1+\beta) m$, podemos remover no máximo $2 \beta m$ vértices de $[A, B]$ para assegurar que o grafo bipartido restante (que continuaremos denotando por $[A, B]$ ) seja conexo na cor vermelha. (Note que não estamos proibindo que este grafo contenha arestas azuis). Agora, qualquer emparelhamento vermelho em $[A, B]$ é conectado. Chamamos de emparelhamento

guloso um emparelhamento onde as arestas são definidas uma a uma e a cada passo simplesmente selecionamos uma nova aresta disjunta (em vértices) das que foram selecionadas até o passo anterior. Seja $b$ o tamanho do maior emparelhamento azul em $[A \cap Q, B \cap Q]$. Teremos dois casos.

Caso 1: $b \leq c m$, para certa constante $c$ que escolheremos adiante.

Removendo no máximo $\mathrm{cm}$ vértices de $A$ e de $B$ e fazendo $p=\beta+2 \beta+c=3 \beta+c$, todas as arestas restantes em $[A, B]$ são vermelhas. Sejam $E_{1}, E_{2}$ emparelhamentos 
vermelhos de tamanho $2 d m$ em $[C, A]$ e em $[C, B]$ respectivamente ( $d$ será determinado $\operatorname{logo}$ abaixo). Selecione $F_{1} \subset E_{1}$ e $F_{2} \subset E_{2}$ tais que $\left|F_{1}\right|=\left|F_{2}\right|=d m$ e $F_{1} \cup F_{2}$ é um emparelhamento (isto é possível mesmo no "pior" caso em que $\left.V\left(E_{1}\right) \cap C=V\left(E_{2}\right) \cap C\right)$. Selecione então um emparelhamento guloso $M$ em $\left[A \backslash V\left(F_{1}\right), B \backslash V\left(F_{2}\right)\right]$. Usando que no máximo $\sqrt{\varepsilon} n \leq 4 \eta m$ arestas estão faltando em cada vértice, $|M| \geq(1-p-4 \eta-d) m$, então

$$
\left|M \cup F_{1} \cup F_{2}\right| \geq(d+1-p-4 \eta) m \geq\left(\frac{1}{4}+\eta\right) n,
$$

desde que

$$
d \geq 8 \eta+3 \beta+c
$$

Então podemos assumir que as arestas vermelhas de $[A, C]$ ou $[B, C]$ podem ser eliminadas após a remoção de no máximo $2 d m=(16 \eta+6 \beta+2 c) m$ vértices. Podemos supor, por simetria, a primeira opção, assim ajustando $p=24 \eta+9 \beta+3 c$ ficamos com todas as arestas de $[A, C]$ na cor azul.

Subcaso 1.a: $[B, C]$ tem emparelhamentos $M_{1}$ e $M_{2}$, vermelho e azul respectivamente, cada um de tamanho $d m$.

Neste caso, podemos assumir que a maioria das arestas de $[A, D]$ são verdes. De fato, suponha que tivéssemos um emparelhamento $M_{3}$ azul de tamanho $d m$ em $[A, D]$. Então $M_{2} \cup M_{3}$ juntamente com um emparelhamento guloso de $\left[A \backslash V\left(M_{3}\right), C \backslash V\left(M_{2}\right)\right]$ seria um emparelhamento azul conectado de tamanho pelo menos $(1-p) m-4 \eta m+d m \geq\left(\frac{1}{4}+\eta\right) n$ desde que

$$
d \geq 8 \eta+p
$$

(O argumento análogo aplica-se caso exista um emparelhamento vermelho de tamanho $d m$ em $[A, D]$ ). O mesmo argumento também mostra que podemos encontrar o emparelhamento azul conectado ou vermelho (de tamanho $\left.\left(\frac{1}{4}+\eta\right) n\right)$ se o subgrafo induzido por $A$ possui um emparelhamento azul ou vermelho de tamanho $\frac{d m}{2}$ desde que

$$
d / 2 \geq 8 \eta+p
$$

Assim, eliminando $2(16 \eta+2 p)+2(8 \eta+p)=48 \eta+6 p$ vértices, e atualizando o valor de $p$, conseguimos com que todas as arestas de $[A, D]$ e todas as arestas dentro de $A$ sejam verdes. Novamente, pelo mesmo argumento, podemos encontrar o emparelhamento procurado (dessa vez na cor verde) se $[C, D]$ ou $[B, D]$ possuir um emparelhamento verde de tamanho $d m$ desde que (4.13) seja verdade (já que dentro de $A$, em particular, existe um emparelhamento verde de tamanho $d m / 2$ ). Então, removendo no máximo $2 d m$ vértices de $B, C, D$ todas as arestas no grafo tripartido $T$ induzido por $B, C, D$ são vermelhas ou azuis - depois de ter ajustado $p$. Aplicando o lema 4.9, encontramos ou uma componente monocromática, digamos vermelha, $C^{*}$ que cobre $V(T)$ quase todo ou duas componentes $C_{1}, C_{2}$, a primeira vermelha e a segunda azul, que cobrem duas partes de $T$ e juntas cobrem a terceira parte. No primeiro caso, 
$\left|C \backslash V\left(C^{*}\right)\right| \leq 6 \sqrt{\varepsilon} n \leq 6 \eta n=24 \eta m$. Logo, um emparelhamento vermelho de tamanho $(p+2 \eta) 4 m$ em $\left[C \cap V\left(C^{*}\right), D\right]$ seria suficiente para estender qualquer emparelhamento guloso de $[A, B]$ a um emparelhamento vermelho conectado do tamanho desejado. Assim, removendo no máximo $24 \eta m+(p+2 \eta) 4 m$ vértices de $C, D$ e ajustando $p$, todas as arestas que sobram em $[C, D]$ são azuis. Finalmente isto implica que não pode haver um emparelhamento azul em $[B, D]$ de tamanho $(p+2 \eta) 4 m$, caso contrário achamos um emparelhamento azul do tamanho adequado estendendo um emparelhamento guloso de $[A, C]$. Finalmente, com uma última remoção e ajuste de $p$, todas as arestas de $[B, D]$ passam a ser vermelhas. Agora, o que encontramos foi uma $\mathrm{EC}_{2}$ cujo parâmetro $\alpha$ é uma combinação linear de $\eta, \beta, c$. Se $C_{1}$ é uma componente vermelha cobrindo dois dos conjuntos $B, C, D$, o mesmo argumento anterior funciona.

Subcaso 1.b: $[B, C]$ não possui emparelhamento azul de tamanho $d m$. (O caso em que $[B, C]$ não possui emparelhamento vermelho de tamanho $d m$ é análogo)

Como a técnica aqui é basicamente a mesma que no caso anterior, não iremos calcular precisamente o valor de $p$. Removendo no máximo $d m$ vértices de $B, C$, todas as arestas de $[B, C]$ se tornam vermelhas. Usando a mesma técnica de antes, um emparelhamento vermelho de tamanho $d m$ em $[C, D]$ ou em $[A, D]$ seria suficiente para encerrar a prova; um emparelhamento azul de tamanho $d m$ em ambos $[C, D]$ e $[A, D]$ teria o papel análogo. Então podemos concluir que pelo menos um entre $[C, D],[A, D]$ (após a remoção de no máximo $2 d m$ vértices de $A, C$ e $D$ ) possui apenas arestas verdes. Se isto vale para ambos então temos uma $\mathrm{EC}_{2}$. Se $[C, D]$ (resp., $[A, D]$ ) possui apenas arestas verdes $[A, D]$ (resp., $[C, D]$ ) possui um emparelhamento azul de tamanho $d m$ então, como no subcaso 1.a, todas as arestas dentro de $C$ (resp., $A$ ) devem ser verdes também. Finalmente concluímos que o grafo tripartido induzido por $A, B, D$ (resp., $(A, B, C))$ é colorido apenas de vermelho ou azul e podemos terminar como no subcaso 1.a.

Para finalizar o caso 1 , temos que definir $d$ como o máximo do valor das duas combinações lineares de $\eta, \beta, c$ definidas implicitamente nos subcasos acima e fazer o mesmo para o valor do parâmetro $\alpha$.

Caso 2: $b \geq \mathrm{cm}$.

Seja $A_{1}=A \cap Q$ e $B_{1}=B \cap Q, A_{2}=A \backslash A_{1}, B_{2}=B \backslash B_{1}$. Usando as hipóteses sobre as ordens desses conjuntos temos que

$$
(1-3 \beta) m-\left|A_{2}\right|+\left|B_{1}\right| \leq\left|A_{1}\right|+\left|B_{1}\right| \leq(1+\beta) m
$$

e as inequações análogas para $B_{2}, A_{1}$ também valem, de onde concluímos que

$$
\left|B_{1}\right| \leq\left|A_{2}\right|+4 \beta m, \quad\left|A_{1}\right| \leq\left|B_{2}\right|+4 \beta m .
$$

Além disso, como $b \geq \mathrm{cm}$, em particular $\left|A_{1}\right|,\left|B_{1}\right| \geq \mathrm{cm}$. Ou seja, nenhum dos conjuntos $A_{i}, B_{i}$ é muito pequeno. 
Suponha que existem emparelhamentos vermelhos $E_{1}, E_{2}$ ambos de tamanho $2 d m$ em $[A, C]$ e em $\left[B_{1}, C\right]$. Tome $F_{1} \subset E_{1}, F_{2} \subset E_{2}$ tais que $\left|F_{1}\right|=\left|F_{2}\right|=d m$ e $F_{1} \cup F_{2}$ é um emparelhamento. Considere o emparelhamento $M=F_{1} \cup F_{2} \cup M_{1} \cup M_{2} \cup M_{3}$ onde $M_{1}, M_{2}$, são emparelhamentos gulosos em $\left[A_{1} \backslash V\left(F_{1}\right), B_{2}\right],\left[B_{1} \backslash V\left(F_{2}\right), A_{2} \backslash V\left(F_{1}\right)\right] \mathrm{e}$ $M_{3}$ é um emparelhamento guloso de $\left[A_{2} \backslash\left(V\left(M_{1}\right) \cup V\left(M_{2}\right)\right), B_{2} \backslash V\left(M_{1}\right)\right]$.

Afirmamos que $M_{1} \cup M_{2} \cup M_{3}$ cobre $A \backslash V\left(F_{1}\right)$ ou $B \backslash V\left(F_{2}\right)$ com um erro de no máximo $(4 \beta+4 \eta) m$. Observe que, por causa de (4.14), $A_{1} \backslash V\left(F_{1}\right)$ é quase coberto por $M_{1}$ (no máximo $4 \beta m$ vértices de $A_{1}$ não são cobertos). Se $A_{2} \backslash V\left(F_{1}\right)$ é coberto por $M_{2}$, a afirmação está provada (com erro $4 \beta m$ ). Caso contrário tome um vértice $x \in A_{2} \backslash V\left(F_{1}\right)$ que não é coberto por $M_{2}$ e, pela definição de $M_{2}, B_{1} \backslash F_{2}$ é quase coberto por $M_{2}(\operatorname{com}$ erro $\eta n=4 \eta m)$. Finalmente, pela definição de $M_{3}$, ou $B_{2}$ é quase coberto por $M_{1} \cup M_{3}$ ou $A_{2} \backslash V\left(F_{1}\right)$ é quase coberto por $M_{2} \cup M_{3}$ (com erro $\eta n=4 \eta m)$. Portanto, a afirmação está provada. Contando o número de arestas em $M_{1} \cup M_{2} \cup M_{3}$ a partir do lado melhor coberto de $[A, B]$, digamos $A$, temos que

$$
\begin{aligned}
|M| & =\left(\left|F_{1}\right|+\left|F_{2}\right|\right)+\left(\left|M_{1}\right|+\left|M_{2}\right|+\left|M_{3}\right|\right) \geq 2 d m+\left|A \backslash V\left(F_{1}\right)\right|-4 \beta m-4 \eta m= \\
& =|A|+d m-4 \beta m-4 \eta m \geq(d+1-7 \beta-4 \eta) m \geq\left(\frac{1}{4}+\eta\right) n
\end{aligned}
$$

desde que

$$
d \geq(8 \eta+7 \beta) m
$$

e portanto encontramos um emparelhamento monocromático conectado do tamanho desejado. Concluímos portando que $[A, C]$ ou $\left[B_{1}, C\right]$ não possui emparelhamento vermelho de tamanho $2 d m$. De maneira análoga, $\left[A_{1}, C\right]$ ou $[B, C]$ não possui emparelhamento vermelho de tamanho $2 d m$. Isto nos dá duas opção para testar.

Subcaso 2.a: $[A, C]$ não possui $2 d m$ arestas vermelhas independentes. (O mesmo argumento vale para $[B, C]$ por simetria.)

Removendo no máximo $2 d m$ vértices de $A, C$ e ajustando $p=3 \beta+2(8 \eta+7 \beta)$ podemos assumir que todas as arestas de $[A, C]$ são azuis. Usando estas arestas e o emparelhamento azul de tamanho pelo menos $\mathrm{cm}$ em $\left[A_{1}, B_{1}\right]$, se existisse um emparelhamento azul de tamanho $d m$ em $[B, C]$ poderíamos achar um emparelhamento azul do tamanho requerido (se $c, d$ são grandes em termos de $\beta$ e $\eta$ ). Logo, após ajustarmos $p$, podemos supor que as arestas de $[B, C]$ são vermelhas. Repetindo o mesmo argumento, segue que as arestas de $[C, D]$ são verdes, e que as arestas dentro de $C$ são verdes, então que as de $[A, D]$ são azuis, as de $[B, D]$ são vermelhas e encontramos uma $\mathrm{EC}_{2}$, desde que $c, d$ sejam combinações lineares adequadas de $\beta, \eta$. O parâmetro $\alpha$ da $\mathrm{EC}_{2}$ é o valor final de $p$ (que também é uma combinação linear de $\beta$ e $\eta$ ).

Subcaso 2.b: nem $\left[A_{1}, C\right]$ nem $\left[B_{1}, C\right]$ possui um emparelhamento vermelho de tamanho $2 d m$.

Seja $B^{*}=B_{1} \cup A_{1}, A^{*}=B_{2} \cup A_{2}$. Repetindo os mesmos passos usuais, todas as arestas de $\left[A_{1}, C\right],\left[B_{1}, C\right]$ são azuis, então $\left[B^{*}, C\right]$ é azul. 
Suponha primeiro que $\left|B^{*}\right|>(1-p-d-2 \eta) m$. Então os passos habituais mostram que podemos assumir que as arestas de $\left[A^{*}, C\right]$ são todas vermelhas, já que com um emparelhamento azul de tamanho $d m$ em $\left[A^{*}, C\right]$ poderíamos terminar a prova. Segue também que todas as arestas de $[C, D]$ são verdes. Com um emparelhamento vermelho de tamanho $d m$ dentro de $C$ também conseguimos terminar. Suponha agora que existe um emparelhamento azul de tamanho $d m$ dentro de $C$. Então um emparelhamento vermelho ou azul de tamanho $d m$ em $\left[B^{*}, D\right]$ nos permitiria construir o emparelhamento desejado na cor vermelha ou azul. Caso contrário, todas as arestas de $\left[B^{*}, D\right]$ são verdes e com isto todas as arestas de $\left[A^{*}, B^{*}\right]$ devem ser vermelhas e temos uma $\mathrm{EC}_{2}$. A conclusão é que todas as arestas dentro de $C$ são verdes. Agora, um emparelhamento verde ou vermelho de tamanho em $d m$ em $\left[B^{*}, D\right]$ seria suficiente. Logo, $\left[B^{*}, D\right]$ deve ser azul, então $\left[A^{*}, D\right]$ é vermelho e novamente temos uma $\mathrm{EC}_{2}$.

Finalmente podemos assumir que $\left|B^{*}\right|=\left|A_{1}\right|+\left|B_{1}\right|<(1-p-d-2 \eta) m$. Afirmamos que ou $\left[A_{2}, C\right]$ ou $\left[B_{2}, C\right]$ é azul. De fato, pelo procedimento usual, suponha que $E_{1} \cup E_{2}$ é um emparelhamento vermelho, tal que $E_{1} \subset\left[A_{2}, C\right], E_{2} \subset\left[B_{2}, C\right],\left|E_{1}\right|=\left|E_{2}\right|=d m$. Como $|A|,|B| \geq(1-p) m \geq\left|A_{1}\right|+\left|B_{1}\right|+d m+2 \eta m$, segue que $\left|A \backslash A_{1}\right|=\left|A_{2}\right| \geq$ $\left|B_{1}\right|+d m+2 \eta m,\left|B \backslash B_{1}\right|=\left|B_{2}\right| \geq\left|A_{1}\right|+d m+2 \eta m$. Portanto, no grafo bipartido $[A, B]$, todos os vértices de $A_{1}$ podem ser gulosamente emparelhados a vértices de $B_{2} \backslash V\left(E_{2}\right)$ formando um emparelhamento vermelho $M_{1}$, todos os vértices de $B_{1}$ podem ser gulosamente emparelhados a vértices de $A_{2} \backslash V\left(E_{1}\right)$ formando um emparelhamento vermelho $M_{2}$. Seja então $M_{3}$ o maior emparelhamento guloso vermelho em $\left[A_{2} \backslash\left(V\left(E_{1}\right) \cup\right.\right.$ $\left.\left.V\left(M_{2}\right)\right), B_{2} \backslash\left(V\left(E_{2}\right) \cup V\left(M_{1}\right)\right)\right]$. Portanto, $M=E_{1} \cup E_{2} \cup M_{1} \cup M_{2} \cup M_{3}$ cobre $A$ ou $B$ com erro no máximo $\eta n=4 \eta m$. Contando o número de arestas de $M$ a partir do lado que está melhor coberto, digamos $A$, segue que

$$
\begin{aligned}
|M| & =\left(\left|E_{1}\right|+\left|E_{2}\right|\right)+\left(\left|M_{1}\right|+\left|M_{2}\right|+\left|M_{3}\right|\right) \geq 2 d m+\left(\left|A \backslash V\left(E_{1}\right)\right|-4 \eta m\right) \geq \\
& \geq|A|+d m-4 \eta m \geq\left(\frac{1}{4}+\eta\right) n
\end{aligned}
$$

desde que

$$
d \geq(p+8 \eta) m
$$

e portanto encontramos um emparelhamento monocromático conectado do tamanho desejado. Logo, a afirmação está provada e, por simetria, assumimos que $\left[A_{2}, C\right]$ é azul (o que implica que $[A, C]$ é azul). Daí, como $\left[B_{1}, C\right]$ também é azul, podemos estender o emparelhamento azul a um do tamanho requerido.

Com isto terminamos a prova do lema 4.11 


\section{Capítulo 5}

\section{Número de Ramsey para três circuitos pares de mesmo tamanho}

Um resultado original deste trabalho, obtido em conjunto com Jozef Skokan [2], é o teorema a seguir.

Teorema 5.1. Existe uma constante $n_{0}$ tal que para $n>n_{0}, n$ par, temos

$$
R\left(C_{n}, C_{n}, C_{n}\right)=2 n
$$

A prova deste resultado segue o mesmo esquema geral do artigo de Gyárfás, Ruszinkó, Sárközy e Szemerédi [17], cujo resultado principal é o teorema 1.4. Contudo, precisamos melhorar alguns dos lemas desse artigo à fim de encontrar um circuito ao invés de apenas um caminho.

Começamos exibindo uma coloração que mostra que vale o limite inferior no teorema 5.1 (i.e., $R\left(C_{n}, C_{n}, C_{n}\right)>2 n-1$ ).

Coloração 5 ( $\left.\mathrm{EC}_{M A X}\right)$. Seja $n$ um inteiro par. Particione os vértices de $K_{2 n-1}$ em conjuntos $A \cup B \cup C \cup D \cup K$ onde $|A|=|B|=|C|=|D|=n / 2-1$ e $|K|=3$. Seja $K=\{r, g, b\}$. Pinte as arestas internas aos conjuntos $A, B, C, D$ de maneira arbitrária, as arestas em $[A, B] \cup[C, D]$ de vermelho, as arestas em $[A, D] \cup[B, C]$ de verde, e as arestas em $[A, C] \cup[B, D]$ de azul. Agora, pinte todas as arestas de $r$ para $A \cup B \cup C \cup D$ de vermelho. De modo semelhante, pinte as arestas de $g$ para $A \cup B \cup C \cup D \cup\{r\}$ de verde. $E$, finalmente, pinte todas as $2 n-2$ arestas incidentes a $b$ de azul.

Lema 5.2. Para todo $n$ par,

$$
R\left(C_{n}, C_{n}, C_{n}\right)>2 n-1 .
$$




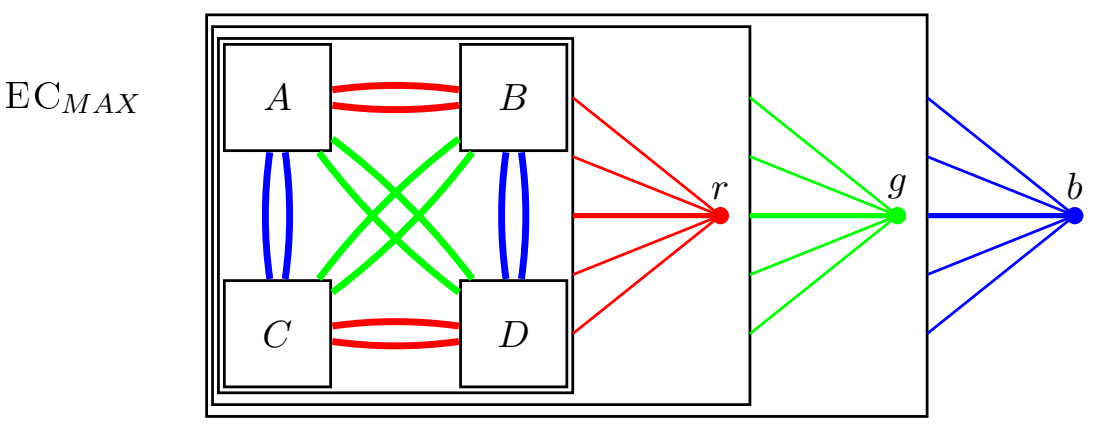

Prova. Basta ver que a coloração $\mathrm{EC}_{M A X}$ definida acima não contém um $C_{n}$ monocromático. Considere então uma $\mathrm{EC}_{M A X}$ de $K_{2 n-1}$ e $A \cup B \cup C \cup D \cup\{r, g, b\}$ como na definição da mesma. É fácil ver que $G=K_{2 n-1} \backslash\{r, g, b\}$ não contém nenhum $C_{n}$ monocromático, já que as componentes conexas de cada um dos grafos $G^{B}, G^{R}, G^{G}$ possuem menos do que $n$ vértices. Também é fácil ver que não existe nenhum $C_{n}$ monocromático que passa por algum dos vértices $r, g, b$.

Para verificar que a cota superior do teorema 5.1 vale, i.e., $R\left(C_{n}, C_{n}, C_{n}\right) \leq 2 n$, para $n$ par suficientemente grade, teremos que provar que toda 3-coloração de um $K_{2 n}$ possui um $C_{n}$ monocromático. A principal ferramenta para provar esta cota é o lema de estabilidade (lema 4.1) de Gyárfás, Ruszinkó, Sárközy e Szemerédi [17], cuja prova exibimos no capítulo 4.

A estratégia que usaremos nesta prova tem se tornado padrão nos últimos anos. A idéia se baseia nos passos a seguir, que serão feitos em detalhes na próxima subseção. Seremos forçados a iniciar a prova escolhendo uma série de parâmetros para que possamos aplicar uma seqüência de lemas nos passos subseqüentes. Neste momento escolhemos também $n_{0}$. Olharemos então para uma 3-coloração $\left(G^{R}, G^{G}, G^{B}\right)$ de um grafo completo $G:=K_{2 n}$, onde $n$ é par e $n>n_{0}$, e aplicaremos o teorema 3.6 aos grafos $G^{R}, G^{G}, G^{B}$ para obter uma partição de $V(G)$. Definiremos um grafo reduzido a partir dessa partição e em seguida uma multicoloração de suas arestas. Aplicaremos então o lema de estabilidade (lema 4.1) para esta multicoloração o que nós dará 4 casos a analisar. Tal multicoloração contém um emparelhamento monocromático conectado de um certo tamanho ou ela é do tipo $\mathrm{EC}_{1}$ ou do tipo $\mathrm{EC}_{2}$ ou do tipo $\mathrm{EC}_{3}$, definidos no início do capítulo 4. No caso do lema nos dar o emparelhamento monocromático conectado, conseguiremos utiliza-lo para encontrar um $C_{n}$ monocromático no grafo original. Nos demais casos, provamos que a coloração original também é do tipo $\mathrm{EC}_{1}$, $\mathrm{EC}_{2}$ ou $\mathrm{EC}_{3}$ e então aplicamos um dos lemas abaixo ao grafo original para concluir que o mesmo possui um $C_{n}$ monocromático. As provas desses lemas serão apresentadas apenas nas seções 5.3 e 5.4, após a prova do teorema 5.1 . 
Lema 5.3. Existe $\alpha_{5.3}>0$ tal que, para todo $\alpha \leq \alpha_{5.3}$ e todo $\delta<\alpha$, existe um inteiro positivo $n_{5.3}$ tal que o seguinte vale para todo $n \geq n_{5.3}$ par: toda 3 -coloração de $K_{2 n}$ do tipo $\mathrm{EC}_{1}(\alpha, \delta)$ contém um $C_{n}$ monocromático.

Lema 5.4. Existe $\alpha_{5.4}>0$ tal que, para todo $\alpha \leq \alpha_{5.4}$ e todo $\delta<\alpha$, existe um inteiro positivo $n_{5.4}$ tal que o seguinte vale para todo $n \geq n_{5.4}$ par: toda 3 -coloração de $K_{2 n}$ do tipo $\mathrm{EC}_{2}(\alpha, \delta)$ contém um $C_{n}$ monocromático.

Lema 5.5. Existe $\mu_{5.5}>0$ tal que, para todo $\mu \leq \mu_{5.5}, c_{1}<1$ e $c_{2}<0,5$, existem $n_{5.5}=n_{5.5}\left(\mu, c_{1}, c_{2}\right)$ e $\delta_{5.5}=\delta_{5.5}\left(\mu, c_{1}, c_{2}\right)$ tais que o seguinte vale para todo $n \geq n_{5.4}$, $n$ par, e $0<\delta \leq \delta_{5.5}$ : toda 3-coloração de $K_{2 n}$ do tipo $\mathrm{EC}_{3}\left(\mu, c_{1}, c_{2}, \delta\right)$ contém um $C_{n}$ monocromático. Ademais, para $c_{1}, c_{2}$ fixos e $\mu_{1}<\mu_{2}$ temos $\delta_{5.5}\left(\mu_{1}, c_{1}, c_{2}\right) \leq$ $\delta_{5.5}\left(\mu_{2}, c_{1}, c_{2}\right)$ e $n_{5.5}\left(\mu_{1}, c_{1}, c_{2}\right) \geq n_{5.5}\left(\mu_{2}, c_{1}, c_{2}\right)$.

Definição 26. Para simplificar futuras referências, iremos definir versões mais fortes das colorações $\mathrm{EC}_{1}, \mathrm{EC}_{2}$ e $\mathrm{EC}_{3}$. Uma coloração é $\mathrm{EC}_{1}$-completa com parâmetro $\alpha$ se ela é uma coloração do tipo $\mathrm{EC}_{1}(\alpha, 0)$, ou seja, $\delta=0$. Neste caso, o que estamos exigindo é que todos os grafos bipartidos envolvidos na segunda condição da definição da coloração $\mathrm{EC}_{1}$ sejam completos. Definimos as colorações $\mathrm{EC}_{2}$-completa e $\mathrm{EC}_{3}$ completa de maneira análoga (ou seja, fixando $\delta=0$ ).

O restante deste capítulo é organizado da seguinte forma: faremos a prova do teorema 5.1 na seção a seguir, usando os lemas 5.3, 5.4 e 5.5. Em seguida mostramos alguns resultados simples que usaremos nas prova desses lemas e finalmente exibimos as provas dos mesmos.

Notação 27. Se uma arestas $x y$ é vermelha dizemos que $y$ é um vizinho vermelho de $x$ (e vice-versa). Denotamos por $N_{R}(x)$ o conjunto dos vizinhos vermelhos de $x$ e definimos $\operatorname{deg}_{R}(x, Y):=\left|N_{R}(x) \cap Y\right|$ (o grau vermelho de $x$ em $Y$ ). Fazemos o análogo para as cores verde e azul.

\subsection{Prova do teorema 5.1}

Prova. Pelo lema 5.2, já temos que para todo $n$ par $R\left(C_{n}, C_{n}, C_{n}\right)>2 n-1$. Resta então provar que existe $n_{0}$ tal que para $n$ par e $n>n_{0}$ temos que $R\left(C_{n}, C_{n}, C_{n}\right) \leq 2 n$. Primeiro temos que definir $n_{0}$ para depois olharmos para uma 3-coloração de $G:=K_{2 n}$ e tentarmos provar que existe um $C_{n}$ monocromático nesta coloração. Mas a escolha de $n_{0}$ depende de inúmeros outros parâmetros que serão usados para aplicar lemas sobre $G$ e sobre um grafo reduzido construído a partir da coloração de $G$. Essa escolha de parâmetros tem que ser feita no parágrafo a seguir, mas em uma primeira leitura é possível saltar este parágrafo, desde que se aceite que as premissas necessárias para aplicar os lemas sejam satisfeitas ao longo da prova. 
Primeiro escolhemos $\alpha_{1}$ e $\mu_{1}$. Num futuro próximo, precisaremos que $\alpha_{1}$ seja pequeno o suficiente para que os lemas 5.3 e 5.4 possam ser aplicados e precisaremos também que $\alpha_{1} \leq 1 / 20$. Definimos então $\alpha_{1}:=\min \left\{\alpha_{5.3}, \alpha_{5.4}, 1 / 20\right\}$. Por sua vez, $\mu_{1}$ é um parâmetro que será usado como entrada para o lema 5.5. Tomamos então $\mu_{1}:=\mu_{5.5}$. Agora escolheremos os valores de parâmetros principais $\eta$ e $\varepsilon$. Passando os valores escolhidos de $\alpha_{1}$ e $\mu_{1}$ como entrada para o lema 4.1, ele nos retorna (independentemente do grafo em que será aplicado) $\varepsilon_{4.1}, \eta_{4.1}$ e $\mu_{4.1}<\mu_{1}$. Tome $\eta:=\eta_{4.1}$. Agora, o lema 5.5 com entrada $\mu=\mu_{4.1} / 2, c_{1}:=0,8, c_{2}:=0,3$ nos devolve $n_{5.5}$ e $\delta_{5.5}$. Tome $\varepsilon$ tal que $\varepsilon<\varepsilon_{4.1}, 2 \varepsilon^{1 / 3}<\delta_{5.5}, \varepsilon<0,01 \varepsilon^{1 / 3}$ e $\varepsilon<g\left(\alpha_{1}, \mu_{4.1}, \eta\right)$, para alguma função $g$ que não iremos calcular explicitamente. Tendo escolhido também $\varepsilon$, o lema 4.1 nos dá $n_{4.1}$, e podemos escolher $m=\max \left\{n_{4.1}, 1 / \varepsilon\right\}$ e $s=3$ para aplicar o lema da regularidade (teorema 3.6) $\operatorname{com}$ parâmetros $\varepsilon, m$ e $s$, que, por sua vez, nos devolve $N_{3.6}$ e $M_{3.6}$.

Finalmente, tome uma 3-coloração $\left(G^{R}, G^{G}, G^{B}\right)$ de $G:=K_{2 n}$ com $n$ par e grande o suficiente, digamos $n=\max \left\{n_{5.3}, n_{5.4}, n_{5.5}, N_{3.6}, 2 M_{3.6} n_{3.3}\right\}$, e aplique o teorema 3.6 para os grafos $G^{R}, G^{G}, G^{B}$ com parâmetros $\varepsilon, m$ e $s$ como acima. Ele nos devolve uma partição $V_{0} \cup V_{1} \cup \ldots \cup V_{t}$ de $V$ tal que $m<t<M_{3.6}$. Considere o grafo reduzido, $H$, desta partição definido da seguinte maneira: os vértices de $H$ são $1, \ldots, t$ e colocamos uma aresta entre os vértices $i$ e $j$ se e somente se $\left(V_{i}, V_{j}\right)$ é um par $\varepsilon$-regular em relação a $G^{R}, G^{G}$ e $G^{B}$. Note que

$$
e(H) \geq(1-\varepsilon)\left(\begin{array}{l}
t \\
2
\end{array}\right)
$$

Defina também uma 3-multicoloração $\left(H^{R}, H^{G}, H^{B}\right)$ de $H$ : coloque a aresta $i j$ em $H^{R}$ (resp., $H^{G}, H^{B}$ ) se $e_{R}\left(V_{i}, V_{j}\right) \geq \frac{\varepsilon^{1 / 3}}{4}\left|V_{i}\right|\left|V_{j}\right|$ (resp., $e_{G}\left(V_{i}, V_{j}\right), e_{B}\left(V_{i}, V_{j}\right) \geq$ $\left.\frac{\varepsilon^{1 / 3}}{4}\left|V_{i}\right|\left|V_{j}\right|\right)$. Aplicando o lema 4.1 a esta coloração de $H$ temos quatro casos.

Caso 1: existe um emparelhamento monocromático conectado $M=\left\{e_{0}, \ldots, e_{t_{1}-1}\right\}$ de tamanho $t_{1} \geq(1 / 4+\eta) t$ em $H$.

Assuma que $M$ é vermelho. Sejam $a_{i}, b_{i}$ as extremidades de $e_{i}$. Então

$$
\left|\bigcup_{i=0}^{t_{1}-1} V_{a_{i}} \cup V_{b_{i}}\right| \geq 2 t_{1} \frac{(1-\varepsilon) 2 n}{t} \geq\left(\frac{1}{2}+2 \eta\right)(1-\varepsilon)(2 n) \geq(1+3 \eta) n
$$

dado que é é pequeno em função de $\eta$ (pois tomamos $\varepsilon<g\left(\alpha, \mu_{4.1}, \eta\right)$ ).

Agora, usamos argumentos padrão de regularidade para construir um circuito monocromático de tamanho $n$ em $G^{R}$. Seja $F$ o subgrafo vermelho conexo minimal de $H$ que contém $M$. Claramente, $F$ é uma árvore. Considere um passeio fechado de comprimento mínimo $W=i_{1} i_{2} \ldots i_{l} i_{1}$ que contém todas as arestas de $M$. Como $F$ é uma árvore, $W$ tem comprimento par. Usando propriedades simples de regularidade, como a proposição 3.2, não é difícil encontrar (gulosamente) em $G^{R}$ um circuito par 
$\tilde{C}=v_{i_{1}} v_{i_{2}} \ldots v_{i_{l}} v_{i_{1}}$ com $v_{i_{j}} \in V_{i_{j}}$ tal que $v_{i_{j}}$ tem pelo menos $\frac{\varepsilon^{1 / 3}}{5}\left|V_{i_{j}}\right|$ vizinhos vermelhos em ambos $V_{i_{j-1}}$ e $V_{i_{j+1}}$, para todo $1 \leq j \leq l$. Contudo, o comprimento de $C$ é $l$, o que é bem menor do que $n$. A idéia agora é substituir as arestas de $M$ em $C$ por caminhos de modo a estender $C$ a um $C_{n}$ vermelho. Daí, para cada aresta $e_{k}$ de $M$ escolhemos um número natural $1 \leq l_{k} \leq\left(1-5 \varepsilon / \varepsilon^{1 / 3}\right)\left|V_{i_{k}}\right|$ de modo que

$$
\sum_{k=0}^{t_{1}-1} 2 l_{k}=n-l
$$

Isto é possível, já que $\sum_{k=0}^{t_{1}-1} 2 l_{k}$ pode ser tão grande quanto $\left(1-5 \varepsilon / \varepsilon^{1 / 3}\right)(1+3 \eta) n=$ $\left(1-5 \varepsilon^{2 / 3}\right)(1+3 \eta) n>(1+2 \eta) n$ e $n-l$ é par. (A desigualdade anterior, novamente segue do fato de que $\varepsilon$ é pequeno em função de $\eta)$. Finalmente, podemos usar o lema 3.3, pois $\left|V_{i}\right| \geq(1-\varepsilon) 2 n / M_{3.6}>n_{3.3}$, uma vez para cada um dos grafos $G\left[V_{a_{k}}, V_{b_{k}}\right]\left(0 \leq k<t_{1}\right)$ e substituir as arestas $v_{a_{k}} v_{b_{k}}$ de $\tilde{C}$ por um caminho de comprimento $2 l_{k}+1$ que começa em $v_{a_{k}}$, termina em $v_{b_{k}}$ e consiste apenas de arestas em $G^{R}\left[V_{a_{k}}, V_{b_{k}}\right]$. Claramente, o resultado disto é um circuito monocromático de comprimento $n$.

Caso 2: $\left(H^{R}, H^{G}, H^{B}\right)$ é uma coloração do tipo $\mathrm{EC}_{1}$ com parâmetro $\alpha_{1} / 2$. Mostraremos que isto implica que $\left(G^{R}, G^{G}, G^{B}\right)$ é uma coloração $\mathrm{EC}_{1}$ com parâmetro $\alpha_{1}$. Considere uma partição $V(H)=A \cup B \cup C \cup D$ que satisfaz as condições de $\mathrm{EC}_{1}$. Olhe para a partição $V(G)=\left(f(A) \cup V_{0}\right) \cup f(B) \cup f(C) \cup f(D)$ onde $f(X):=\bigcup_{i \in X} V_{i}$. Primeiro note que

$$
\left|f(A) \cup V_{0}\right|,|f(B)|,|f(C)|,|f(D)| \geq\left(1-\frac{\alpha_{1}}{2}\right) \frac{t}{4} \frac{(1-\varepsilon)(2 n)}{t} \geq\left(1-\alpha_{1}\right) \frac{2 n}{4} .
$$

Resta provar que a segunda condição da $\mathrm{EC}_{1}$ também é satisfeita para esta partição, com a 3-coloração $\left(G^{R}, G^{G}, G^{B}\right)$ original. Mostraremos como obter uma cota superior para o número de arestas entre $f(A) \cup V_{0}$ e $f(B)$ que não estão em $G^{R}$ que neste caso é igual a $G^{R^{*}}$ (lembre da definição 18). As demais cotas seguem de maneira análoga.

Há no máximo $\frac{\alpha_{1}}{2}|A||B|$ arestas $i j \in[A, B]$ tais que $i j \notin H^{R^{*}}$. Mesmo que, para cada aresta $i j$ desse tipo, $\left[V_{i}, V_{i}\right]$ possua apenas arestas verdes e azuis, ao todo teremos no máximo $\frac{\alpha_{1}}{2}|f(A)||f(B)|$ arestas não vermelhas entre esses pares de conjuntos. Por outro lado, para cada aresta $i j \in[A, B] \operatorname{com} i j \in H^{R^{*}}$, temos que $i j \notin H^{G} \cup H^{B}$. Então, o número de arestas não vermelhas em $G\left[V_{i}, V_{i}\right]$ é $\left|\left(G^{G} \cup G^{B}\right)\left[V_{i}, V_{j}\right]\right| \leq 2\left(\frac{\varepsilon^{1 / 3}}{4}\left|V_{i}\right|\left|V_{j}\right|\right)$. Portanto,

$$
\left|\left(G^{G} \cup G^{B}\right)[f(A), f(B)]\right| \leq \frac{\alpha_{1}}{2}|f(A)||f(B)|+\frac{\varepsilon^{1 / 3}}{2}|f(A)||f(B)| .
$$

Agora, mesmo que todas as arestas de $\left[V_{0}, f(B)\right]$ não sejam vermelhas, temos que a densidade de arestas não vermelhas em $\left[f(A) \cup V_{0}, f(B)\right]$ é no máximo

$$
\frac{\frac{\alpha_{1}}{2}|f(A)||f(B)|+\frac{\varepsilon^{1 / 3}}{2}|f(A)||f(B)|+\left|V_{0}\right||f(B)|}{\left|f(A) \cup V_{0}\right||f(B)|} \leq \frac{\alpha_{1}}{2}+\frac{\varepsilon^{1 / 3}}{2}+\frac{\left|V_{0}\right|}{|f(A)|}<\alpha_{1},
$$


dado que $\left|V_{0}\right| /|f(A)| \leq \frac{\varepsilon(2 n)}{n / 5}=10 \varepsilon$, pois $\frac{1}{4}-\alpha_{1} \geq 1 / 5$. Então $G^{R}\left[f(A) \cup V_{0}, f(B)\right]$ é $\left(1-\alpha_{1}\right)$-denso.

Agora basta usar o lema 5.3 para encontrar o $C_{n}$ monocromático em $G$.

Caso 3: $\left(H^{R}, H^{G}, H^{B}\right)$ é uma coloração do tipo $\mathrm{EC}_{2}$ com parâmetro $\alpha_{1} / 2$. Do mesmo modo que no caso anterior, podemos mostrar que $\left(G^{R}, G^{G}, G^{B}\right)$ é uma $\mathrm{EC}_{2}$ com parâmetro $\alpha_{1}$. Neste caso usamos o lema 5.4 para encontrar o $C_{n}$ monocromático em $G$. Isto completa a prova.

Caso 4: $\left(H^{R}, H^{G}, H^{B}\right)$ é uma coloração do tipo $\mathrm{EC}_{3}$ com parâmetros $\mu=\mu_{4.1}$, $c_{1}=0,7, c_{2}=0,2$ e $\delta=\varepsilon^{1 / 3}$. Como no caso 2, consideramos $V(H)=A \cup B \cup C \cup D$, partição que satisfaz as condições de $\mathrm{EC}_{3}$, e olhamos para a partição $V(G)=(f(A) \cup$ $\left.V_{0}\right) \cup f(B) \cup f(C) \cup f(D)$ onde $f(X):=\bigcup_{i \in X} V_{i}$. Provaremos que esta partição define uma $\mathrm{EC}_{3}$ com parâmetros $\mu=(1-\varepsilon) \mu_{4.1}, c_{1}=0,8, c_{2}=0,3$, e $\delta=2 \varepsilon^{1 / 3}$. Primeiro é trivial que

$$
|f(D)| \geq \mu_{4.1} \frac{t}{4} \frac{(1-\varepsilon)(2 n)}{t} \geq(1-\varepsilon) \mu_{4.1} \frac{2 n}{4} .
$$

Vemos também que

$$
\left|f(A) \cup V_{0}\right|,|f(B)|,|f(C)| \geq\left(1-0,7 \mu_{4.1}\right) \frac{t}{4} \frac{(1-\varepsilon)(2 n)}{t} \geq\left(1-0,8(1-\varepsilon) \mu_{4.1}\right) \frac{2 n}{4} .
$$

Também que

$$
|A|-|B| \geq \mu_{4.1} \frac{t}{4} \Rightarrow|f(A)|-|f(B)| \geq \mu_{4.1} \frac{t}{4} \frac{(1-\varepsilon)(2 n)}{t}=(1-\varepsilon) \mu_{4.1} \frac{2 n}{4},
$$

e que o análogo vale se trocarmos $B$ por $C$ ou $D$, de onde segue que

$$
\left|f(A) \cup V_{0}\right| \geq|f(A)| \geq \max \{|f(B)|,|f(C)|,|f(D)|\}+(1-\varepsilon) \mu_{4.1} \frac{2 n}{4} .
$$

E finalmente

$$
\left|f(A) \cup V_{0} \cup D\right| \leq\left(1+0,2 \mu_{4.1}\right) \frac{t}{2} \frac{2 n}{t}+\varepsilon(2 n) \leq\left(1+0,3(1-\varepsilon) \mu_{4.1}\right) \frac{2 n}{2} .
$$

Ademais, podemos obter as condições de densidade de modo análogo ao caso 2. Sendo assim, basta usar o lema 5.5 para terminar a prova. Podemos fazer isso já que $(1-\varepsilon) \mu_{4.1}<\mu_{4.1}=\mu_{5.5},(1-\varepsilon) \mu_{4.1}>\mu_{4.1} / 2$ e $\delta=2 \varepsilon^{1 / 3}<\delta_{5.5}\left(\mu_{4.1} / 2,0,8,0,3\right)$.

\subsection{Caminhos e circuitos em grafos densos}

A prova do teorema 5.1 terminou, exceto pelos lemas 5.3, 5.4 e 5.5. Esta seção prepara a prova desses lemas. Durante tais provas, precisaremos encontrar caminhos ou circuitos 
longos em grafos densos diversas vezes. Os resultados que precisamos para isto são bastante simples, mas resolvemos organizá-los aqui para melhor clareza e referência.

O primeiro lema é um fato bastante conhecido. Veja, por exemplo, a página 107 de [3].

Lema 5.6. Seja $G_{n}$ um grafo de ordem $n$ que não possui um caminho de tamanho $k \geq 1$. Então o número de arestas de $G_{n}$ é no máximo $\frac{k-1}{2} n$. Ademais, se o número de arestas de $G_{n}$ é igual a $\frac{k-1}{2} n$, então $G_{n}$ é a união de grafos completos de ordem $k$.

O lema seguinte é semelhante ao lema 3.3, porém bem mais simples pois, apesar de que não temos um par $\varepsilon$-regular, a densidade de arestas é muito alta.

Lema 5.7. São dados um natural $n$ par, um real $\beta<1 / 4$ e um grafo bipartido em classes $A$ e $B$ tais que $|A| \geq|B|$. Suponha ainda que, para todo $v \in A, \operatorname{deg}(v, B) \geq$ $(1-\beta)|B|$, e, para todo $v \in B, \operatorname{deg}(v, A) \geq 2$. Temos então que

a) dados $u, v \in A$, para todo $2 \leq k \leq(1-2 \beta)|B|$ existe um caminho de ordem $2 k+1$ começando em $u$ e terminando em $v$;

b) dados $u \in A, v \in B$, para todo $2 \leq k \leq(1-2 \beta)|B|$ existe um caminho de ordem $2 k$ começando em $u$ e terminando em $v$.

Prova. Para provar o item a), escolha $k+1$ pontos $a_{1}, \ldots, a_{k+1} \in A$ tais que $a_{1}=u$, $a_{k+1}=v$. É fácil construir um caminho $P_{k+1}=a_{1} b_{1} a_{2} b_{2} \ldots b_{k} a_{k+1}$, tal que $b_{i} \in B$ para $1 \leq i \leq k$. Uma maneira de fazer isto é escolhendo os $b_{i}^{\prime} s$ indutivamente de maneira gulosa, ou seja, suponha que para certo $l \leq k$ já construímos $P_{l}=a_{1} b_{1} \ldots b_{l-1} a_{l}$. Basta então escolher $b_{l}$ como qualquer vértice na vizinhança comum de $a_{l}$ e $a_{l+1}$ em $B \backslash V\left(P_{l}\right)$. Tal vértice existe pois

$$
\left|\left(N\left(a_{l+1}\right) \cap N\left(a_{l}\right)\right) \backslash V\left(P_{l}\right)\right| \geq(|B|-2 \beta|B|)-(l-1) \geq 1,
$$

já que

$$
l \leq k \leq(1-2 \beta)|B| .
$$

Para o item b), basta começar tomando um vizinho $w$ de $v$ em $A$ tal que $w \neq u$, e então aplicar o item anterior para encontrar um caminho de tamanho $2 k-1$ entre $u$ e $w$ (apenas tomando o cuidado de não usar $v$ neste caminho).

O próximo lema é sobre uma situação muito específica que acontecerá na seção seguinte.

Lema 5.8. Sejam $\tilde{A}$ e $\tilde{B}$ conjuntos tais que $|\tilde{A}|=|\tilde{B}|$. Suponha que, para $\beta<1 / 3$, existem partições $A \cup A^{\prime}$ de $\tilde{A}$ e $B \cup B^{\prime}$ de $\tilde{B}$ tais que $\left|A^{\prime}\right| \leq \beta|\tilde{A}|,\left|B^{\prime}\right| \leq \beta|\tilde{B}|,[A, B]$ é um grafo bipartido completo, e que, para todo $a \in A^{\prime}$, temos $\operatorname{deg}(a, B) \geq 2 \beta|\tilde{B}|$ e, para todo $b \in B^{\prime}$, temos $\operatorname{deg}(b, A) \geq 2 \beta|\tilde{A}|$. Então o grafo bipartido $[\tilde{A}, \tilde{B}]$ é hamiltoniano. 
Prova. Não é difícil se convencer de que este grafo é hamiltoniano e há várias maneiras de provar isso. Optamos aqui por uma prova construtiva, que não usa nenhum teorema forte como Hall ou Dirac, pois logo mais precisaremos relaxar a condição sobre os graus de alguns elementos de $A^{\prime}$ e $B^{\prime}$. Nossa prova ainda funcionará após tal relaxação.

Primeiro note que, transferindo alguns vértices de $A$ para $A^{\prime}$ ou de $B$ para $B^{\prime}$, podemos supor que $\left|A^{\prime}\right|=\left|B^{\prime}\right| \leq \beta|\tilde{B}|=\beta|\tilde{A}|$.

Em um primeiro passo, enumere todos os vértices de $A^{\prime}$, digamos $a_{1}, \ldots, a_{k}$, e todos os vértices de $B^{\prime}$, digamos $b_{1}, \ldots, b_{k}$. Para cada $a_{i}(1 \leq i \leq k)$ escolha dois vizinhos $x_{i, 1}, x_{i, 2} \in B$, de modo que $x_{1,1}, \ldots, x_{k, 1}, x_{1,2}, \ldots, x_{k, 2}$ sejam dois a dois distintos (isto pode ser feito facilmente de maneira gulosa). Do mesmo modo escolha dois vizinhos $y_{i, 1}, y_{i, 2} \in A$ de cada $b_{i}$, todos distintos entre si.

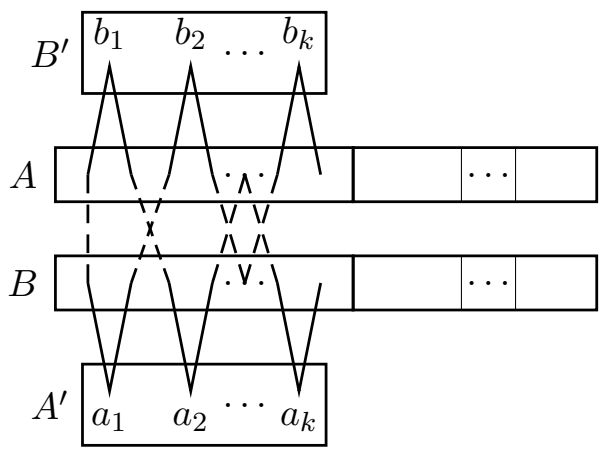

Agora, para construir um caminho, basta usar as arestas do grafo completo $[A, B]$. Conecte $x_{1,1}$ a $y_{1,1} \mathrm{e}$, para $1 \leq i \leq k-1$, conecte $x_{i, 2}$ a $y_{i+1,1}$ e $y_{i, 2}$ a $x_{i+1,1}$. O resultado é um caminho $P$ de ordem $6 k$ começando em $x_{k, 2}$ e terminando em $y_{k, 2}$. Estender $P$ a um circuito hamiltoniano de $[\tilde{A}, \tilde{B}]$ é trivial, já que os vértices que não pertencem a $P$ formam um grafo bipartido completo com classes de vértices de mesma ordem.

Observação. Note que, no primeiro passo da prova, quando precisamos escolher os vértices $x_{i, 1}, x_{i, 2}$ dois a dois distintos, a condição de que, para todo $v \in A^{\prime}, \operatorname{deg}(v, B) \geq$ $2 \beta|\tilde{B}|$ é até forte demais. Seria suficiente supor que existe uma ordenação dos vértices de $A^{\prime}, a_{1}, \ldots, a_{k}$, tal que, para todo $i, \operatorname{deg}\left(a_{i}, B\right) \geq 2 i$. Em particular, no caso em que $A^{\prime}$ possui um número constante $c$ de vértices cujo grau em $B$ de cada um é pelo menos $2 c$, e que a condição original $\operatorname{deg}(v, B) \geq 2 \beta n / 2$ é satisfeita para os demais vértices em $A^{\prime}$ ainda podemos usar a mesma construção para encontrar o circuito hamiltoniano.

Finalmente, o último lema de que precisaremos é uma versão do lema anterior onde $\tilde{A}$ e $\tilde{B}$ possuem ordens diferentes, mas temos arestas no interior de $A$ para nos ajudar.

Lema 5.9. Sejam $n$ e $t$ naturais, $n$ par, e $\tilde{A}, \tilde{B}$ conjuntos tais que $|\tilde{A}|=n / 2+t$, $|\tilde{B}|=n / 2-t$. Suponha que existem partições $A \cup A^{\prime}$ de $\tilde{A}$ e $B \cup B^{\prime}$ de $\tilde{B}$ tais que $\left|A^{\prime}\right| \leq$ 
$\beta(n / 2-t),\left|B^{\prime}\right| \leq \beta(n / 2-t)$, para $\beta<1 / 4$, e $[A, B]$ é um grafo bipartido completo (como no lema anterior). Suponha ainda que para todo $a \in A^{\prime}$ temos $\operatorname{deg}(a, B) \geq 2 \beta|\tilde{B}|$ e para todo $b \in B^{\prime}$ temos $\operatorname{deg}(b, A) \geq 2 \beta|\tilde{B}|+2 t+1$. Finalmente, suponha que existe um caminho $P$ de ordem $2 t+1$ com todos seus vértices pertencentes a $A$. Então o grafo induzido por $\tilde{A} \cup \tilde{B}$ é hamiltoniano.

Prova. Seja $P$ o caminho de ordem $2 t+1$ em $A$ e sejam $u, v$ as extremidades de $P$. Considere o grafo $[(\tilde{A} \backslash P) \cup\{u\}, \tilde{B}]$ e remova dele as arestas entre $u$ e $B^{\prime}$. Veja que $|(\tilde{A} \backslash P) \cup\{u\}|=|B|=n / 2-t$. Assim, a mesma construção do lema anterior nos dá um circuito hamiltoniano neste grafo e, como $u$ não possui vizinhos em $B^{\prime}$, os vizinhos de $u$ neste circuito pertencem a $B$. Considere um desses vizinhos, digamos $u^{\prime}$, e substitua a aresta $u u^{\prime}$ do circuito pelo caminho $u P v u^{\prime}$. Obtemos então um circuito hamiltoniano do grafo original.

Observação. Vale para este lema uma observação semelhante à que segue o lema anterior.

\subsection{Prova dos lemas 5.3 e 5.5}

Prova. Faremos as provas dos lemas 5.3 e 5.5 em um passo só, já que elas são quase idênticas.

Considere uma 3-coloração de $K_{2 n}$ tal que existe uma partição $A \cup B \cup C \cup D$ de $V\left(K_{2 n}\right)$ que, ou satisfaz as condições da $\mathrm{EC}_{1}$ com parâmetros $\alpha$ e $\delta$, ou satisfaz as condições da $\mathrm{EC}_{3}$ com parâmetros $\mu, c_{1}, c_{2}$ e $\delta$, como no enunciado do lema. Ou seja, admitimos que $\alpha$ e $\mu$ são pequenos e que $\delta$ é suficientemente pequeno em relação a $\mu$ e $c$.

Chamaremos um vértice de $A$ de ruim se ele não possui grau alto para $B, C$ ou $D$ nas cores apropriadas. Mais precisamente, um vértices $v \in A$ é ruim se

$\operatorname{deg}_{R}(v, B)<\left(1-\delta^{1 / 2}\right)|B|, \quad \operatorname{deg}_{B}(v, C)<\left(1-\delta^{1 / 2}\right)|C| \quad$ ou $\operatorname{deg}_{G}(v, D)<\left(1-\delta^{1 / 2}\right)|D|$.

Veja que, há menos do que $2 \delta^{1 / 2}|A|$ vértices em $A$ tais que $\operatorname{deg}_{R}(v, B)<\left(1-\delta^{1 / 2}\right)|B|$, caso contrário, pelo menos $2 \delta|A||B|$ arestas de $[A, B]$ não seriam vermelhas. Fazendo o mesmo para $[A, C]$ e $[A, D]$, segue que o número de vértices ruins de $A$ é, ao todo, no máximo $6 \delta^{1 / 2}|A|$. Do mesmo modo, simultaneamente, classificamos alguns vértices de $B, C$ e $D$ como ruins. Seja $E_{1}$ o conjunto dos vértices ruins. Temos então que $\left|E_{1}\right| \leq 6 \delta^{1 / 2}(|A|+|B|+|C|+|D|)=12 \delta^{1 / 2} n$. Denote então, $A_{1}=A \backslash E_{1}, B_{1}=$ $B \backslash E_{1}, C_{1}=C \backslash E_{1}$ e $D_{1}=D \backslash E_{1}$.

Feito isso, todos os vértices em $A_{1}$ são adjacentes a pelo menos

$$
\left(1-7 \delta^{1 / 2}\right)|B| \geq\left(1-7 \delta^{1 / 2}\right)\left|B_{1}\right| \geq\left(1-\delta^{1 / 3}\right)\left|B_{1}\right|
$$


vértices em $B_{1}$ por arestas vermelhas. Assim, o lema 5.7, $\operatorname{com} \beta=\delta^{1 / 3}$, nos dá caminhos entre $A_{1}$ e $B_{1}$ que saturam quase todos os vértices do menor desses conjuntos. O mesmo vale para os outros pares de conjuntos nas cores correspondentes.

Concluímos então que não existem duas arestas paralelas (independentes) da cor vermelha entre $A_{1}$ e $C_{1}$ : se $a_{1}, c_{1}$ e $a_{2}, c_{2}$ fossem duas arestas desse tipo, poderíamos achar um $a_{1}, a_{2}$-caminho $P$ em $\left[A_{1}, B_{1}\right]$ e um $c_{1}, c_{2}$-caminho $Q$ em $\left[C_{1}, D_{1}\right]$ que podem ser unidos através das duas arestas para obter um circuito $C_{k}=a_{1} P a_{2} c_{2} Q c_{1} a_{1}$ vermelho de tamanho $k$. Veja que, pelo lema lema 5.7, o tamanho de $P$ pode ser tão grande quanto $2\left(1-2 \delta^{1 / 3}\right) \min \left\{\left|A_{1}\right|,\left|B_{1}\right|\right\}$ e o de $Q$ tão grande quanto $2\left(1-2 \delta^{1 / 3}\right) \min \left\{\left|C_{1}\right|,\left|D_{1}\right|\right\}$. Assim, o tamanho $k$ do circuito obtido pode ser tão grande quanto

$$
2\left(1-2 \delta^{1 / 3}\right)\left(\min \left\{\left|A_{1}\right|,\left|B_{1}\right|\right\}+\min \left\{\left|C_{1}\right|,\left|D_{1}\right|\right\}+1\right) .
$$

Aqui precisamos analisar o caso em que temos uma $\mathrm{EC}_{1}$ e o caso em que temos uma $\mathrm{EC}_{3}$ separadamente. O primeiro é fácil, como $\left|A_{1}\right|,\left|B_{1}\right|,\left|C_{1}\right|,\left|D_{1}\right| \geq\left(1-\alpha-24 \delta^{1 / 2}\right) n / 2$, $k$ pode ser tão grande quanto

$$
2\left(1-2 \delta^{1 / 3}\right)\left(1-\alpha-24 \delta^{1 / 2}\right) n \geq 2\left(1-2 \alpha^{1 / 3}\right)\left(1-\alpha^{1 / 3}\right) n \geq\left(1-3 \alpha^{1 / 3}\right)(2 n)>n .
$$

No segundo caso, a cota é um pouco mais crítica, $k$ poderá ser tão grande quanto

$$
2\left(1-2 \delta^{1 / 3}\right)\left(\left(1-c_{1} \mu\right) n / 2+\mu n / 2-12 \delta^{1 / 2} n\right)>\left(1-2 \delta^{1 / 3}\right)\left(1+\left(1-c_{1}\right) \mu-24 \delta^{1 / 2}\right) n,
$$

mas isto também é maior do que $n$, já que $\delta$ é pequeno em relação a $\mu$ e $c_{1}<1$.

Isto implica que não existem arestas vermelhas em $\left[A_{1}, C_{1}\right]$, com exceção de no máximo uma estrela. Aplicando o mesmo argumento para os outros conjuntos e cores, concluímos que esta é uma coloração $\mathrm{EC}_{1}$-completa ou $\mathrm{EC}_{3}$-completa, módulo no máximo 12 estrelas das quais podemos nos livrar removendo no máximo 12 vértices de $A_{1} \cup B_{1} \cup C_{1} \cup D_{1}$. Sejam $A_{2}, B_{2}, C_{2}, D_{2}, E_{2}$ os conjuntos obtidos após removermos esses vértices e os colocarmos em $E_{1}$.

Temos então que, o grafo induzido por $A_{2} \cup B_{2} \cup C_{2} \cup D_{2}$ forma uma coloração $\mathrm{EC}_{1}$-completa ou $\mathrm{EC}_{3}$-completa.

Agora, vamos redistribuir os vértices de $E_{2}$ para os conjuntos $A_{2}, B_{2}, C_{2}$ e $D_{2}$ mantendo a estrutura da coloração e os graus altos nas cores apropriadas.

Primeiro, classificaremos alguns vértices especiais de $E_{2}$. Chamamos um vértice de especial do tipo $R 1$ se $v \in E_{2}$ e

$$
\operatorname{deg}_{R}\left(v, A_{2}\right), \operatorname{deg}_{R}\left(v, C_{2}\right) \geq 4
$$

$\mathrm{Ou}$

$$
\operatorname{deg}_{R}\left(v, B_{2}\right), \operatorname{deg}_{R}\left(v, D_{2}\right) \geq 4 .
$$


Afirmação 5.10. O conjunto $E_{2}$ possui no máximo um vértice do tipo $R 1$, ou conseguimos achar um $C_{n}$ monocromático.

Prova. Suponha que existem dois vértices $v_{1}$ e $v_{2}$ do tipo $R 1$. Assuma, sem perda da generalidade, que $\operatorname{deg}_{R}\left(v_{1}, A_{2}\right), \operatorname{deg}_{R}\left(v_{1}, C_{2}\right) \geq 4$ e sejam $a_{1} \in A_{2}, c_{1} \in C_{2}$ vizinhos vermelhos de $v_{1}$. Daí, se $\operatorname{deg}_{R}\left(v_{2}, A_{2}\right), \operatorname{deg}_{R}\left(v_{2}, C_{2}\right) \geq 4$, então existem $a_{2} \in A_{2}, c_{2} \in C_{2}$ vizinhos vermelhos de $v_{2}$ e distintos de $a_{1}, c_{1}$. Pelo lema 5.7, do mesmo modo que acima, podemos encontrar um caminho vermelho $P$ de comprimento par entre $a_{1}$ e $a_{2}$ em $\left[A_{2}, B_{2}\right]$ e um caminho vermelho $Q$ de comprimento par entre $c_{1}$ e $c_{2}$ em $\left[C_{2}, D_{2}\right]$ tais que $v_{1} a_{1} P a_{2} v_{2} c_{2} Q c_{1} v_{1}$ é o circuito de tamanho $n$ que estamos procurando. No caso em que $\operatorname{deg}_{R}\left(v_{2}, B_{2}\right), \operatorname{deg}_{R}\left(v_{2}, D_{2}\right) \geq 4$ procedemos de maneira semelhante: tome $b_{2} \in B_{2}, d_{2} \in D_{2}$ vizinhos vermelhos de $v_{2}$, encontre um caminho ímpar $P$ de $a_{1}$ para $b_{2}$ com arestas em $\left[A_{2}, B_{2}\right]$ e um caminho ímpar $Q$ de $c_{1}$ para $d_{2}$ com arestas em $\left[C_{2}, D_{2}\right]$ tais que $v_{1} a_{1} P b_{2} v_{2} d_{2} Q c_{1} v_{1}$ é monocromático e tem comprimento $n$.

Observação. Para esta prova, a definição que demos de vértice do tipo $R 1$ é até forte demais: seria suficiente classificar um vértice como $R 1$ se $\operatorname{deg}_{R}\left(v, A_{2}\right), \operatorname{deg}_{R}\left(v, C_{2}\right) \geq 2$ ou $\operatorname{deg}_{R}\left(v, B_{2}\right), \operatorname{deg}_{R}\left(v, D_{2}\right) \geq 2$. Mas, posteriormente, precisaremos da definição mais forte.

De modo análogo, dizemos que $v \in E_{2}$ é do tipo $R 2 \operatorname{se} \operatorname{deg}_{R}\left(v, A_{2}\right), \operatorname{deg}_{R}\left(v, D_{2}\right) \geq 4$ ou $\operatorname{deg}_{R}\left(v, B_{2}\right), \operatorname{deg}_{R}\left(v, C_{2}\right) \geq 4$. Pelo mesmo argumento acima, existe no máximo um vértice do tipo $R 2$. Dizemos que um vértice é do tipo $R *$ se ele é do tipo $R 1$ ou $R 2$. É imediato verificar que um vértice de $E_{2}$ que satisfaz $\operatorname{deg}_{R}\left(v, A_{2} \cup B_{2}\right) \geq 7$ e $\operatorname{deg}_{R}\left(v, C_{2} \cup\right.$ $\left.D_{2}\right) \geq 7$ é do tipo $R *$. Podemos então definir, de maneira análoga, os tipos $B 1, B 2$, $B *, G 1, G 2$ e $G *$ :

$v$ é $B 1: \operatorname{se} \operatorname{deg}_{B}\left(v, A_{2}\right), \operatorname{deg}_{B}\left(v, B_{2}\right) \geq 4 \mathrm{ou}_{\operatorname{deg}_{B}}\left(v, C_{2}\right), \operatorname{deg}_{B}\left(v, D_{2}\right) \geq 4$;

$v$ é $B 2: \operatorname{sedeg}_{B}\left(v, A_{2}\right), \operatorname{deg}_{B}\left(v, D_{2}\right) \geq 4 \mathrm{ou}_{\operatorname{deg}_{B}}\left(v, B_{2}\right), \operatorname{deg}_{B}\left(v, C_{2}\right) \geq 4$;

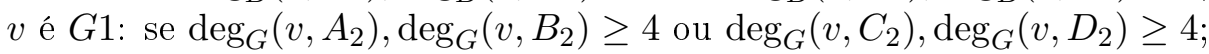

$v$ é $G 2: \operatorname{sedeg}_{G}\left(v, A_{2}\right), \operatorname{deg}_{G}\left(v, C_{2}\right) \geq 4 \mathrm{oudeg}_{G}\left(v, B_{2}\right), \operatorname{deg}_{G}\left(v, D_{2}\right) \geq 4$.

Usando o análogo da afirmação 5.10 para as outras cores, podemos assumir que existem ao todo no máximo 6 vértices dos tipos $R *, B *$ e $G *$. Seja $F$ o conjuntos desses vértices e $E_{2}^{\prime}=E_{2} \backslash F$. A seguir, particionaremos $E_{2}^{\prime}$ em $A_{2}^{\prime} \cup B_{2}^{\prime} \cup C_{2}^{\prime} \cup D_{2}^{\prime}$.

Para todos os vértices $v \in E_{2}^{\prime}$, temos $\operatorname{deg}_{R}\left(v, A_{2} \cup B_{2}\right) \leq 6$ ou $\operatorname{deg}_{R}\left(v, C_{2} \cup D_{2}\right) \leq 6$. Ademais, $\operatorname{deg}_{B}\left(v, A_{2} \cup C_{2}\right) \leq 6$ ou $\operatorname{deg}_{B}\left(v, B_{2} \cup D_{2}\right) \leq 6$. Também, $\operatorname{deg}_{G}\left(v, A_{2} \cup\right.$ $\left.D_{2}\right) \leq 6$ ou $\operatorname{deg}_{G}\left(v, B_{2} \cup C_{2}\right) \leq 6$. Tome qualquer vértice $v \in E_{2}^{\prime}$. Suponha, sem perda de generalidade, que $\operatorname{deg}_{R}\left(v, C_{2} \cup D_{2}\right) \leq 6$ e $\operatorname{deg}_{B}\left(v, B_{2} \cup D_{2}\right) \leq 6$. Então $\operatorname{deg}_{G}\left(v, D_{2}\right) \geq\left|D_{2}\right|-12$, donde $\operatorname{deg}_{G}\left(v, B_{2} \cup C_{2}\right) \leq 6$. Finalmente isto implica que $\operatorname{deg}_{B}\left(v, C_{2}\right) \geq\left|C_{2}\right|-12$ e $\operatorname{deg}_{R}\left(v, B_{2}\right) \geq\left|B_{2}\right|-12$. Neste caso, colocaremos $v$ no conjunto $A_{2}^{\prime}$. (Nos outros possíveis casos colocaríamos $v$ em um dos conjunto $B_{2}^{\prime}, C_{2}^{\prime}$ 
ou $D_{2}^{\prime}$ ). Após fazer o procedimento acima para cada vértice de $E_{2}^{\prime}$, defina $\tilde{A}_{2}=A_{2} \cup A_{2}^{\prime}$, $\tilde{B}_{2}=B_{2} \cup B_{2}^{\prime}, \tilde{C}_{2}=C_{2} \cup C_{2}^{\prime}$ e $\tilde{D}_{2}=D_{2} \cup D_{2}^{\prime}$.

Lembre-se que $\left|E_{2}^{\prime}\right| \leq\left|E_{1}\right|+12 \leq 13 \delta^{1 / 2} n$. Ademais, $\left|\tilde{A}_{2} \cup \tilde{B}_{2} \cup \tilde{C}_{2} \cup \tilde{D}_{2}\right| \geq 2 n-|F| \geq$ $2 n-6$. Assim, se no grafo original tínhamos uma $\mathrm{EC}_{3}$, sabemos que o maior desses conjuntos é $\tilde{A}_{2}$. Se tínhamos uma $\mathrm{EC}_{1}$, podemos assumir o mesmo sem perda da generalidade. Veja que $\left|\tilde{A}_{2}\right| \geq n / 2-1$. Se dois dos conjuntos $\tilde{A}_{2}, \tilde{B}_{2}, \tilde{C}_{2}$ e $\tilde{D}_{2}$, digamos $\tilde{A}_{2}$ e $\tilde{B}_{2}$, têm pelo menos $n / 2$ vértices, pelo lema 5.8 , existe um circuito vermelho $C_{n}$ com arestas em $\left[\tilde{A}_{2}, \tilde{B}_{2}\right]$. Sendo assim, podemos assumir que $\left|\tilde{B}_{2}\right|=n / 2-b,\left|\tilde{C}_{2}\right|=n / 2-c$ e $\left|\tilde{D}_{2}\right|=n / 2-d$, onde $b, c, d>0$. Defina $\left|\tilde{A}_{2}\right|=n / 2+a$, onde $a \geq 0$ ou $a=-1$, e $|F|=f$. Então

$$
a+f=b+c+d
$$

Agora, temos dois casos.

Caso 1: $a \geq 0$.

Afirmação 5.11. Existe em $A_{2}$ um caminho vermelho de tamanho $2 b$, ou um caminho azul de tamanho $2 c$, ou um caminho verde de tamanho $2 d$.

Prova. Suponha que nenhum desses caminhos existe. Então o lema 5.6 nos dá um cota superior para o número de arestas em $A_{2}$.

$$
\left(\frac{\left|A_{2}\right|-1}{2}\right)\left|A_{2}\right| \leq\left(\frac{2 b-1}{2}+\frac{2 c-1}{2}+\frac{2 d-1}{2}\right)\left|A_{2}\right| .
$$

De onde concluímos que

$$
\frac{n}{2}+a-13 \delta^{1 / 2} n-1 \leq 2(b+c+d)-3 .
$$

E, usando (5.2), temos que $a \geq n / 2-13 \delta^{1 / 2} n-2 f+2$. E temos uma cota para a ordem do conjunto $A$ da partição original,

$$
|A| \geq\left|\tilde{A}_{2}\right|-13 \delta^{1 / 2} n \geq n-26 \delta^{1 / 2} n-2 f+2 .
$$

Mas isto é uma contradição: no caso de termos uma $\mathrm{EC}_{1},|A|=(2 n)-|B \cup C \cup D| \leq$ $n / 2+3 \alpha<n-26 \delta^{1 / 2} n-2 f$; no caso de termos uma $\mathrm{EC}_{3}$, as cotas para $|A \cup D| \mathrm{e}$ $|D|$ implicam que $|A| \leq\left(1+\left(c_{2}-0,5\right) \mu\right) n<n-26 \delta^{1 / 2} n-2 f$, dado que $c_{2}<0,5$ e $\delta$ é pequeno em relação a $\mu$. Isto completa a prova da afirmação.

Suponha então que existe um caminho $P$ verde de tamanho $2 d$, ou seja, ordem $2 d+1$, em $A_{2}$. Os outros casos são análogos, na verdade, até um pouco mais fáceis. Se $d \leq a$ podemos achar um $C_{n}$ verde em $\left[\tilde{A}_{2}, \tilde{D}_{2}\right]$ usando o lema 5.9. Basta nos restringirmos a um subconjunto $\hat{A}_{2}$ de $\tilde{A}_{2}$ com $n / 2+d$ vértices que contenha $P$. As condições para aplicar o lema $5.9 \mathrm{em}\left[\hat{A_{2}}, \tilde{D}_{2}\right]$ são garantidas pela escolha dos parâmetros (em particular, veja que $\left|A_{2}^{\prime}\right|$ e $\left|D_{2}^{\prime}\right|$ são pequenos em relação a $\left.\left|D_{2}\right|\right)$. 
Então podemos supor que $d>a$.

Para cada vértice $v \in F$ olhe para a cor que mais aparece nas arestas de $v$ para $A_{2} \backslash P$. Se existem $d-a$ vértices para os quais esta cor é verde podemos adicionar estes vértices ao conjunto $D^{\prime} \subset \tilde{D}_{2}$. Feito isto, ficaríamos com $\left|\tilde{A}_{2}\right|=n / 2+a,\left|\tilde{B}_{2}\right|=$ $n / 2-b+(b-a)=n / 2-a$ e poderíamos usar achar um $C_{n}$ como acima. Veja que, neste caso, precisamos observar que $d-a \leq f \leq 6$, e usar a observação que segue o lema 5.9 para achar tal circuito. Então existem no máximo $b-a-1$ vértices desse tipo em $F$. Se existem $b$ vértices para os quais esta cor é vermelho, podemos adicionar estes vértices ao conjunto $B^{\prime} \subset \tilde{B}_{2}$ e encontrar um $C_{n}$ vermelho em $\tilde{A}_{2} \cup \tilde{B}_{2}$ usando o lema 5.8 , já que após adicionar os vértices a $\tilde{B}_{2}$ teríamos $\left|\tilde{B}_{2}\right| \geq n / 2$. Então existem no máximo $b-1$ vértices em $F$ tais que esta cor é vermelha. Pelo mesmo motivo existem no máximo $c-1$ vértices em $F$ tais que esta cor é azul.

Contudo, agora temos que

$$
|F| \leq(b-a-1)+(c-1)+(d-1)=|F|-3,
$$

uma contradição.

Caso 2: $a=-1$.

Veja que $\left(\left|\tilde{B}_{2}\right|,\left|\tilde{C}_{2}\right|,\left|\tilde{D}_{2}\right|,|F|\right)$ deve assumir um dos valores abaixo.

$$
\begin{aligned}
& (n / 2-1, n / 2-1, n / 2-1,4) \\
& (n / 2-1, n / 2-1, n / 2-2,5) \\
& (n / 2-1, n / 2-1, n / 2-3,6) \\
& (n / 2-1, n / 2-2, n / 2-2,6)
\end{aligned}
$$

Note que agora, as ordens dos conjuntos $\tilde{A}_{2}, \tilde{B}_{2}, \tilde{C}_{2}, \tilde{D}_{2}$ são basicamente as mesma. Sendo assim, a nossa $\mathrm{EC}_{3}$ também é uma $\mathrm{EC}_{1}$.

Vamos primeiro resolver o caso

$$
\left(\left|\tilde{B}_{2}\right|,\left|\tilde{C}_{2}\right|,\left|\tilde{D}_{2}\right|,|F|\right)=(n / 2-1, n / 2-1, n / 2-1,4) .
$$

Lembre-se que todos os vértices de $F$ são especiais. Como $|F|=4$ dois deles devem ser especiais na mesma cor, digamos que $u$ seja do tipo $R 1$ e $v$ do tipo $R 2$. Podemos assumir, sem perda da generalidade, que $\operatorname{deg}_{R}\left(u, A_{2}\right), \operatorname{deg}_{R}\left(u, C_{2}\right) \geq 4$ e que $\operatorname{deg}_{R}\left(v, A_{2}\right), \operatorname{deg}_{R}\left(v, D_{2}\right) \geq 4$. Mas, neste caso, podemos adicionar $u$ ao conjunto $D_{2}^{\prime}$ e $v$ ao conjunto $C_{2}^{\prime}$ e então achar um $C_{n}$ vermelho em $G\left[\tilde{C}_{2}, \tilde{D}_{2}\right]$ usando o lema 5.8 e a observação que o segue.

Para os demais casos precisaremos usar a afirmação a seguir. 
Afirmação 5.12. Se existem dois vértices do tipo $R *$ (resp., $B *, G *$ ) e existe uma aresta vermelha (resp., azul, verde) dentro de algum dos conjuntos $A, B, C, D$, então conseguimos encontrar um $C_{n}$ vermelha (resp., azul, verde).

Prova. Suponha que existem dois vértices do tipo $R *$, digamos $u$ do tipo $R 1$ e $v$ do tipo $R 2$. Como $A_{2} \cup B_{2} \cup C_{2} \cup D_{2}$ induz uma $\mathrm{EC}_{1}$-completa, usando os vértices $u \mathrm{e}$ $v$, mesmo sem usar arestas internas aos conjuntos $A_{2}, B_{2}, C_{2}, D_{2}$, é possível encontrar circuitos de tamanho $k$ para todo $k$ ímpar entre 7 e $2 n-4 \alpha^{1 / 3}$. Se existe uma aresta interna a um dos conjuntos $A_{2}, B_{2}, C_{2}, D_{2}$ podemos facilmente usá-la e conseguir formar também circuitos vermelhos de tamanho par de quase todos os tamanhos. Em particular, conseguimos encontrar um $C_{n}$ vermelho.

Veja portanto que, se $|F|=6$, deve existir um $C_{n}$ monocromático. De fato, como existem no máximo dois vértices especiais de cada cor, a única maneira de termos $|F|=6$ seria com dois vértices do tipo $R *$, dois do tipo $B *$ e dois do tipo $G *$. Portanto, usando a afirmação acima, não há como colorir as arestas internas aos conjuntos $A_{2}$, $B_{2}, C_{2}$ e $D_{2}$ sem que exista um $C_{n}$ monocromático. Agora, o único caso que sobra é

$$
\left(\left|\tilde{B}_{2}\right|,\left|\tilde{C}_{2}\right|,\left|\tilde{D}_{2}\right|,|F|\right)=(n / 2-1, n / 2-1, n / 2-2,5) .
$$

Construímos um grafo auxiliar $H$ com conjunto de vértices $V(H)=F \cup\{X, Y, Z, W\}$, onde $X, Y, Z, W$ representam os conjuntos $A_{2}, B_{2}, C_{2}, D_{2}$ em alguma ordem. E, com certo abuso de notação, se $X$ representa o conjunto $A_{2}$ usamos $\tilde{X}$ e $X^{\prime}$ para representar $\tilde{A}_{2}$ e $A_{2}^{\prime}$ respectivamente. Todo par de vértices de $H$, exceto os da forma $\{u, v\} \subset F$, é uma aresta. A seguir, definimos uma 3-multicoloração de $H$. As arestas $\{U, V\}$ tais que $U, V \in\{X, Y, Z, W\}$ recebem uma única cor: a mesma das arestas do grafo completo $G[U, V]$; as arestas $u V$ tais que $u \in F$ e $V \in\{X, Y, Z, W\}$ recebem cor vermelha (resp., azul, verde) se $\operatorname{deg}_{R}(u, V) \geq 4$ (resp., $\operatorname{deg}_{B}(u, V) \geq 4, \operatorname{deg}_{G}(u, V) \geq 4$ ).

Já que $|F|=5$ e pela simetria das cores, podemos assumir que $F$ possui dois vértices do tipo $R *$, dois do tipo $B *$ e um do tipo $G *$. Chame estes vértices de $r_{1}, r_{2}, b_{1}, b_{2}$ e $g$ respectivamente. Usando a afirmação 5.12, podemos assumir que todas as arestas dentro dos conjuntos $A_{2}, B_{2}, C_{2}, D_{2}$ são verdes. Agora, como ainda não escolhemos qual dos conjuntos $\tilde{X}, \tilde{Y}, \tilde{Z}, \tilde{W}$ é $\tilde{D}_{2}$, ou seja, qual deles é o que tem ordem $n / 2-2$, podemos assumir sem perda da generalidade que $r_{1} X, r_{1} Y, r_{2} X, r_{2} W$ são vermelhas. Daí, usando que $b_{1}$ e $b_{2}$ são do tido $B *$, pelo menos uma das colorações representadas na figura seguinte ocorre em $H \backslash g$. 


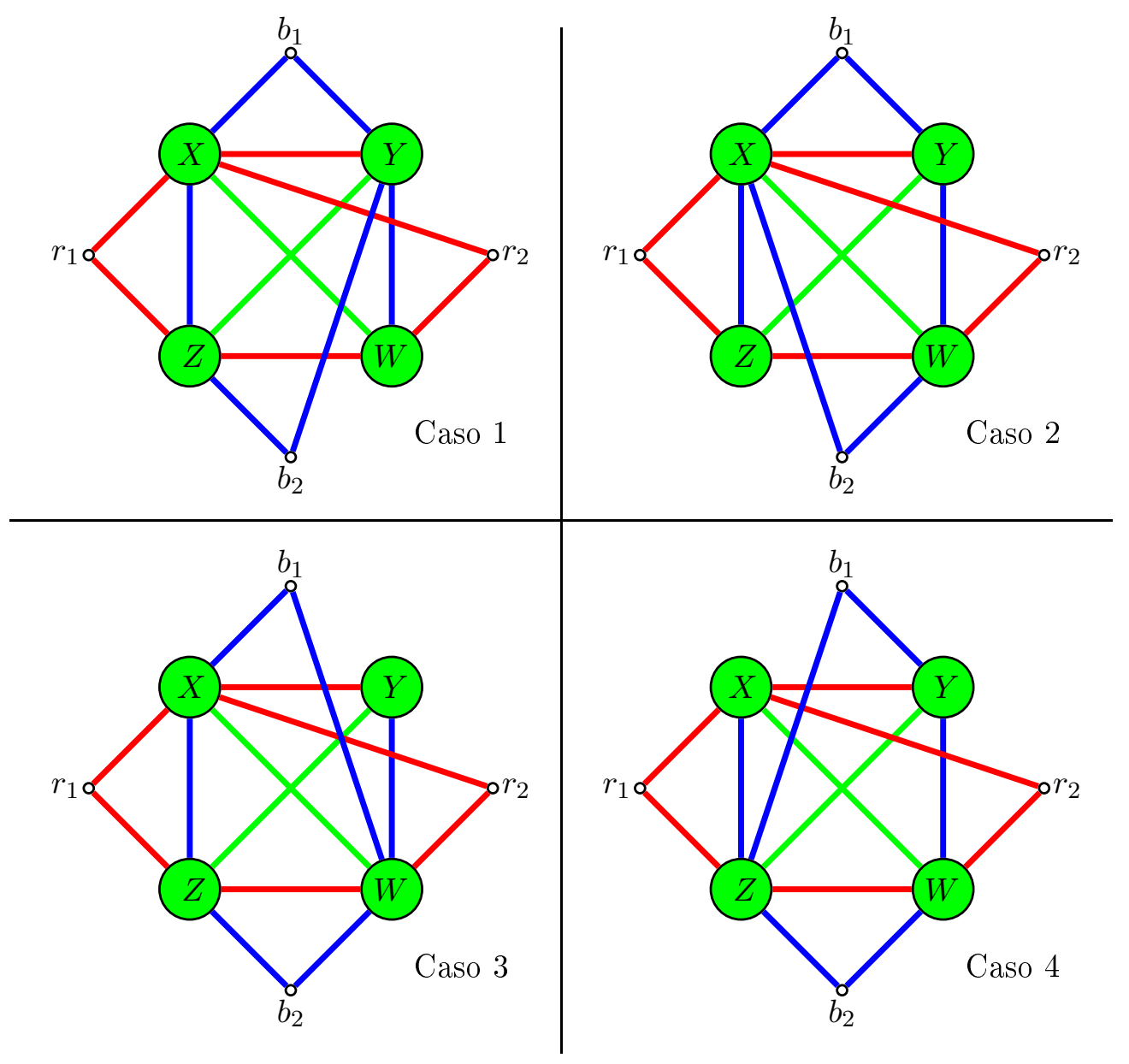

Se em qualquer das colorações $D_{2}=X$ ou $D_{2}=Y$, então podemos adicionar $r_{1}$ a $W^{\prime}$ e $r_{2}$ a $Z^{\prime}$ e depois encontrar um $C_{n}$ vermelho em $G[\tilde{Z}, \tilde{W}]$, usando o lema 5.8. Nas colorações 1 e 3 se $D_{2}=W$ poderíamos adicionar $b_{1}$ a $X^{\prime}$ e $b_{2}$ a $Z^{\prime}$ e depois encontrar um $C_{n}$ azul em $G[\tilde{X}, \tilde{Z}]$, também usando o lema 5.8. Do mesmo modo, nos casos 2 e 4 , se $D_{2}=Z$, podemos encontrar um $C_{n}$ azul em $G[\tilde{Y}, \tilde{W}]$. As outras quatro possibilidades precisam de um pouco mais de trabalho.

Caso 1 e $D_{2}=Z$ : Se a aresta $\left\{b_{2}, W\right\}$ fosse azul então o vértice $b_{2}$ seria do tipo $B 1$. Mas, neste caso, teríamos dois vértices do tipo $B 1$ em $F$ e isto nos daria um $C_{n}$. Se a aresta $\left\{b_{2}, W\right\}$ fosse vermelha então poderíamos adicionar os vértices $b_{2} \mathrm{e}$ $r_{2}$ ao conjunto $Z^{\prime}$ e o vértice $r_{1}$ a $W^{\prime}$ e depois encontrar um $C_{n}$ vermelho em $G[Z, W]$, usando o lema 5.8. Portanto, $\left\{b_{2}, W\right\}$ deve ser exclusiva da cor verde. Um argumento semelhante mostra que $\left\{b_{1}, W\right\}$ deve ser exclusiva da cor verde. Mas agora podemos adicionar $b_{1}$ e $b_{2}$ ao conjunto $X^{\prime}$. Depois disso teremos $|X|=n / 2+1$ e $|W|=n / 2-1$. Mas, lembre-se que as arestas dentro de $X$ são todas verdes, então podemos usar o lema 5.9 para achar um $C_{n}$ verde. Isto encerra este caso.

Caso 2 e $D_{2}=W$ : Agora, a aresta $\left\{b_{2}, Y\right\}$ não pode ser azul (pois neste caso 
teríamos dois vértices do tipo $B 1$ ) e não pode ser vermelha (pois poderíamos adicionar $b_{2}$ a $X^{\prime}$ e $r_{2}$ a $Y^{\prime}$ e depois achar um $C_{n}$ vermelho). Portanto, $\left\{b_{2}, Y\right\}$ deve ser verde. Do mesmo modo a aresta $\left\{r_{2}, Z\right\}$ não pode ser vermelha (pois teríamos dois vértices do tipo $R 1$ ) nem azul (pois poderíamos adicionar $b_{2}$ a $Z^{\prime}$ e $r_{2}$ a $X^{\prime}$ e depois encontrar um $C_{n}$ azul em $\left.G[\tilde{X}, \tilde{Z}]\right)$. Portanto, $\left\{b_{2}, Y\right\}$ deve ser verde, mas, neste caso, podemos adicionar $r_{2}$ a $Y^{\prime}$ e $b_{2}$ a $Z^{\prime}$ e depois encontrar $C_{n}$ verde em $G[\tilde{Y}, \tilde{Z}]$

Os outros casos são quase idênticos, então apenas indicamos para quais arestas precisamos olhar.

Caso 3 e $D_{2}=Z$ : Primeiro $\left\{b_{2}, Y\right\}$ deve ser verde. Segundo $\left\{r_{1}, Y\right\}$ deve ser verde. Também $\left\{b_{1}, Y\right\}$ deve ser verde. Então podemos encontrar um $C_{n}$ verde em $G[\tilde{Y}, \tilde{Z}]$ após adicionar $b_{1}, b_{2}$ e $r_{1}$ ao conjunto $Z^{\prime}$.

Caso 4 e $D_{2}=W$ : Primeiro, $\left\{b_{2}, Y\right\}$ deve ser verde. Também $\left\{r_{1}, Y\right\}$ deve ser verde. Então podemos achar um $C_{n}$ verde em $G[\tilde{Y}, \tilde{Z}]$ após adicionar $b_{2}$ e $r_{1}$ ao conjunto $Z^{\prime}$.

\subsection{Prova do lema 5.4}

Prova. Considere uma 3-coloração de $K_{2 n}$ e seja $V\left(K_{2 n}\right)=A \cup B \cup C \cup D$ uma partição que satisfaz as condições da $\mathrm{EC}_{2}$.

Como na prova do lema anterior, primeiro removemos de $A$ todos os vértices que não possuem grau alto em $B, C$ ou $D$ nas cores apropriadas, então todos os vértices de $B$ que não possuem grau alto em $A, C$ ou $D$ nas cores apropriadas. Então todos os vértices de $C$ e $D$ que não possuem grau alto em $A \cup B$ na cor apropriada. Mais precisamente, um vértice $v \in A$ é removido se

$\operatorname{deg}_{R}(v, B)<\left(1-\alpha^{1 / 2}\right)|B|, \quad \operatorname{deg}_{G}(v, C)<\left(1-\alpha^{1 / 2}\right)|C| \quad$ ou $\operatorname{deg}_{B}(v, D)<\left(1-\alpha^{1 / 2}\right)|D|$.

Pelas condições de densidade em $\mathrm{EC}_{1}$ segue que o número de tais vértices excepcionais em $A$ é no máximo $6 \alpha^{1 / 2}|A|$. Um vértice $v \in B$ é removido se

$\operatorname{deg}_{R}(v, A)<\left(1-\alpha^{1 / 2}\right)|A|, \quad \operatorname{deg}_{G}(v, C)<\left(1-\alpha^{1 / 2}\right)|C| \quad$ ou $\operatorname{deg}_{B}(v, D)<\left(1-\alpha^{1 / 2}\right)|D|$.

Um vértice $v \in C$ é removido se

$$
\operatorname{deg}_{G}(v, A \cup B)<\left(1-\alpha^{1 / 2}\right)(|A|+|B|),
$$

e finalmente um vértice $v \in D$ é removido se

$$
\operatorname{deg}_{B}(v, A \cup B)<\left(1-\alpha^{1 / 2}\right)(|A|+|B|) .
$$


Ao todo, estes somam no máximo $12 \alpha^{1 / 2} n$ vértices excepcionais. Como antes, colocamos estes vértices em um novo conjunto chamado $E_{1}$. E, como antes, definimos $A_{1}=A \backslash E_{1}, B_{1}=B \backslash E_{1}, C_{1}=C \backslash E_{1}, D_{1}=D \backslash E_{1}$.

Agora, todos os vértices em $A_{1}$ são adjacentes a pelo menos $\left(1-7 \alpha^{1 / 2}\right)|B| \geq$ $\left(1-7 \alpha^{1 / 2}\right)\left|B_{1}\right| \geq\left(1-\alpha^{1 / 3}\right)\left|B_{1}\right|$ vértices em $B_{1}$ através de arestas vermelhas e o análogo vale para os outros conjuntos e cores. Se $\left|C_{1}\right| \geq n / 2$ é fácil achar um $C_{n}$ monocromático gulosamente em $\left[C_{1}, A_{1} \cup B_{1}\right]$. De modo análogo é fácil acabar se $\left|D_{1}\right| \geq n / 2$. Logo, suponha que $\left|C_{1}\right|,\left|D_{1}\right|<n / 2$. Sejam $\left|C_{1}\right|=\frac{n}{2}-c$ e $\left|D_{1}\right|=\frac{n}{2}-d$, onde $c, d>0$.

Afirmamos que não existe um caminho verde em $A_{1}$ (nem em $B_{1}$ ) de ordem $2 c+1$ assim como não existe caminho azul em $A_{1}$ (ou em $B_{1}$ ) de ordem $2 d+1$. Suponha que $P$ é um tal caminho em $A_{1}$, com ordem $2 c+1$ e extremidades $a_{1}$ e $a_{2}$. Note que $c$ é muito pequeno, certamente menor que $\alpha^{1 / 3} n / 2$.

Como todos os vértices de $A_{1} \cup B_{1}$ têm grau verde alto em $C_{1}$, podemos achar $c_{1} \neq c_{2} \in C_{1}$ tais que as arestas $a_{1} c_{1}$ e $a_{2} c_{2}$ são verdes. Agora, quaisquer dois vértices em $C_{1}$ têm pelo menos $\left|A_{1} \cup B_{1}\right|-2 \alpha^{1 / 3}\left|A_{1} \cup B_{1}\right|-2 k_{C}>\left|C_{1}\right|$ vizinhos verdes em comum em $A_{1} \cup B_{1} \backslash V(P)$. Daí, podemos de maneira gulosa encontrar um caminho verde $P^{\prime}$, começando em $c_{1}$ e terminando em $c_{2}$ que não passa por $V(P)$ e passa por todos os vértices de $C_{1}$. Então $P \cup P^{\prime}$ é nosso $C_{n}$ monocromático.

Pelo lema 5.6, $A_{1}$ (resp., $\left.B_{1}\right)$ contém no máximo $(2 c-1)\left|A_{1}\right| / 2$ (resp., $\left.(2 c-1)\left|B_{1}\right| / 2\right)$ arestas verdes e no máximo $(2 d-1)\left|A_{1}\right| / 2$ (resp., $\left.(2 d-1)\left|B_{1}\right| / 2\right)$ arestas azuis. Agora, removemos de $A_{1}$ e $B_{1}$ todos os vértices adjacentes a mais que $\alpha^{1 / 6} n$ vértices de $A_{1} \cup B_{1}$ através de arestas verdes ou azuis e os colocamos em $E_{1}$. Chamamos os novos conjuntos de $A_{2}, B_{2}, C_{2}, D_{2}$ e $E_{2}$ (veja que $C_{2}:=C_{1}$ e $D_{2}:=D_{1}$, mas trocamos os índices de todos os conjuntos para manter a notação simples). Note que removemos no máximo $4 \alpha^{1 / 6} n$ vértices, assim $\left|E_{2}\right| \leq 5 \alpha^{1 / 6} n$. Feito isto, o que obtemos é que o grafo $G^{R}\left[A_{2} \cup B_{2}\right]$, induzido pelas arestas vermelhas de $A_{2} \cup B_{2}$, tem grau mínimo $\left|A_{2} \cup B_{2}\right|-5 \alpha^{1 / 6} n$, ou seja, é quase completo.

Estamos chegando ao ponto final. Sejam $n_{A B}=n-\left|A_{2} \cup B_{2}\right|, n_{C}=n / 2-\left|C_{2}\right|$, e $n_{D}=n / 2-\left|D_{2}\right|$. Se $n_{A B}<1$, então estamos feitos já que $G^{R}\left[A_{2} \cup B_{2}\right]$ é hamiltoniano, por Dirac. Logo, suponha que $n_{A B} \geq 1$.

Suponha agora que existem $k=n_{C}<\alpha^{1 / 6} n$ vértices em $E_{2}$, cada um com pelo menos $n / 16$ vizinhos verdes em $A_{2} \cup B_{2}$. Sejam $e_{1}, \ldots, e_{k}$ tais vértices. Como $e_{i}$ tem pelo menos $n / 16$ vizinhos verdes em $A_{2} \cup B_{2}$, podemos escolher para cada $e_{i}$ dois desses vizinhos $a_{i}, b_{i}$ de modo que $a_{1}, b_{1}, a_{2}, b_{2}, \ldots, a_{k}, b_{k}$ sejam dois a dois distintos.

Daí, tomamos um vizinho verde $c_{1} \in C_{2}$ de $a_{1}$, e um vizinho verde $c_{k+1} \in C_{2}$ de $b_{k}$, e para cada par de vértices $b_{i-1}, a_{i}, \operatorname{com} i=2, \ldots, k$, tomamos um de seus vizinhos verdes $c_{i} \in C_{2}$ em comum. Novamente, os $c_{i}$ 's podem ser escolhidos dois a dois distintos já que quaisquer dois vértices de $A_{2} \cup B_{2}$ têm pelo menos $\left|C_{2}\right|-2 \alpha^{1 / 6} n / 2>2 k$ vizinhos 
verdes em $C_{2}$ em comum. Podemos também encontrar de maneira gulosa um $c_{1}, c_{k+1^{-}}$ caminho verde que não passa pelos vértices $a_{i}, b_{i}, c_{i}$ 's e satura todos os demais vértices de $C_{2}$, já que todos os pares de vértices em $C_{2}$ têm uma vizinhança comum grande em $A_{2} \cup B_{2}$. Com isto, temos um $C_{n}$ verde.

Daí, resta o caso em que há menos que $n_{C}<\alpha^{1 / 6} n$ vértices em $E_{2}$ que possuem grau verde maior ou igual a $n / 16$ em $A_{2} \cup B_{2}$. Do mesmo modo, podemos assumir que há menos que $n_{D}<\alpha^{1 / 6} n$ vértices em $E_{2}$ que possuem grau azul maior ou igual a $n / 16$ em $A_{2} \cup B_{2}$.

Então, existem pelo menos $2 n-\left|A_{2} \cup B_{2}\right|-\left|C_{2}\right|-\left|D_{2}\right|-\left(n_{C}-1\right)-\left(n_{D}-1\right)>n-\mid A_{2} \cup$ $B_{2} \mid=n_{A B}$ vértices em $E_{2}$, cada um com pelo menos $\left|A_{2} \cup B_{2}\right|-2(n / 16)>2\left|A_{2} \cup B_{2}\right| / 3$ vizinhos vermelhos em $A_{2} \cup B_{2}$. Seja $F$ um conjunto de $n_{A B}$ vértices desse tipo.

Assim o grafo $G^{R}\left[A_{2} \cup B_{2} \cup F\right]$, induzido pelas arestas vermelhas de $A_{2} \cup B_{2} \cup F$, é hamiltoniano (por Dirac) e tem $n$ vértices. Logo, ele contém um $C_{n}$ vermelho. 


\section{Referências Bibliográficas}

[1] L. Babai, M. Simonovits, and J. Spencer, Extremal subgraphs of random graphs, Journal of Graph Theory 14 (1990), no. 5, 599-622.

[2] F. Benevides and J. Skokan, The 3-colored Ramsey number of even cycles, working notes, 2007.

[3] B. Bollobás, Modern graph theory, Springer-Verlag, New York, 1998.

[4] J. A. Bondy and P. Erdős, Ramsey numbers for cycles in graphs, Journal of Combinatorial Theory, Series B 14 (1973), 46-54.

[5] V. Chvátal, V. Rödl, E. Szemerédi, and W. T. Trotter, Jr., The Ramsey number of a graph with bounded maximum degree, Journal of Combinatorial Theory, Series B 34 (1983), no. 3, 239-243.

[6] E.J. Cockayne and P.J. Lorimer, The Ramsey number for stripes, J. Austral. Math. Soc. Ser. A 19 (1975), 252-256.

[7] Reinhard Diestel, Graph theory, third ed., Graduate Texts in Mathematics, vol. 173, Springer-Verlag, Berlin, 2005.

[8] P. Erdős and M. Simonovits, An extremal graph problem, Acta Mathematica Academiae Scientiarum Hungaricae 22 (1971/1972), 275-282.

[9] R.J. Faudree and R.H. Schelp, Path Ramsey numbers in multicolorings, Journal of Combinatorial Theory, Series B 19 (1975), 150-160.

[10] A. Figaj and T. Łuczak, The Ramsey number for a triple of long even cycles, Journal of Combinatorial Theory, Series B (2006), InPress.

[11] Z. Füredi and A. Gyárfás, Covering t-element sets by partitions, European Journal of Combinatorics 12 (1991), 483-489.

[12] Z. Füredi, O. Pikhurko, and M. Simonovits, The Turán density of the hypergraph $\{a b c, a d e, b d e, c d e\}$, Electronic Journal of Combinatorics 10 (2003), Research Paper 18, 7 pp. (electronic). 
[13] _ On triple systems with independent neighbourhoods, Combinatorics, Probability and Computing 14 (2005), no. 5-6, 795-813.

[14] Z. Füredi and M. Simonovits, Triple systems not containing a Fano configuration, Combinatorics, Probability and Computing 14 (2005), no. 4, 467-484.

[15] L. Geréncser and A. Gyárfás, On Ramsey-type problems, Ann. Univ. Sci. Budapest, Eôtvôs Sect. Math. 10 (1967), 167-170.

[16] R. L. Graham, B. L. Rothschild, and J. H. Spencer, Ramsey theory, second ed., John Wiley \& Sons Inc., New York, 1990, A Wiley-Interscience Publication.

[17] A. Gyárfás, M. Ruszinkó, G. N. Sárközy, and E. Szemerédi, Three color Ramsey numbers for paths, Combinatorica 27 (2007), no. 3, 35-69.

[18] P. Keevash and B. Sudakov, The Turán number of the Fano plane, Combinatorica 25 (2005), no. 5, 561-574.

[19] Y. Kohayakawa, M. Simonovits, and J. Skokan, The 3-colored Ramsey number of odd cycles, Proceedings of Brazilian Symposium on Graphs and Combinatorics (GRACO2005), Angra dos Reis, vol. 19, 2005, pp. 397-402.

[20] G. Károlyi and V. Rosta, Generalized and geometric Ramsey numbers for cycles, Theoretical Computer Science 263 (2001), 87-98.

[21] L. Lovász and M. Simonovits, On the number of complete subgraphs of a graph, Proceedings of the Fifth British Combinatorial Conference (Univ. Aberdeen, 1975) (Winnipeg, Man.) (Aberdeen), Utilitas Math., 1976, Congressus Numerantium, No. XV., pp. 431-441.

[22] _ On the number of complete subgraphs of a graph. II, Proceedings of the Fifth British Combinatorial Conference (Univ. Aberdeen, Aberdeen, 1975) (Winnipeg, Man.), Studies in pure mathematics (Basel), Birkhäuser, 1983, pp. 459-495.

[23] T. Euczak, $R\left(C_{n}, C_{n}, C_{n}\right) \leq(4+o(1)) n$, Journal of Combinatorial Theory, Series B 75 (1999), 174-187.

[24] S. P. Radziszowski, Small Ramsey numbers, Electronic Journal of Combinatorics 1 (1994), Dynamic Survey 1, 30 pp. (electronic).

[25] K. F. Roth, On certain sets of integers, J. London Math. Soc. 28 (1953), 104-109.

[26] M. Simonovits, A method for solving extremal problems in graph theory, stability problems, Theory of Graphs (Proc. Colloq., Tihany, 1966) (New York), Academic Press, 1968, pp. 279-319.

[27] E. Szemerédi, On sets of integers containing no four elements in arithmetic progression, Acta Math. Acad. Sci. Hungar. 20 (1969), 89-104. 
[28] - On sets of integers containing no $k$ elements in arithmetic progression, Acta Arith. 27 (1975), 199-245, Collection of articles in memory of Juriu Vladimirovič Linnik.

[29] Endre Szemerédi, Regular partitions of graphs, Problèmes combinatoires et théorie des graphes (Colloq. Internat. CNRS, Univ. Orsay, Orsay, 1976), CNRS, Paris, 1978, pp. 399-401.

[30] Paul Turán, Eine Extremalaufgabe aus der Graphentheorie, Mat. Fiz. Lapok 48 (1941), 436-452. 